\title{
CHARACTERIZATION OF THE ACTIVE SITE OF CYSTATHIONINE $\gamma$-LYASE FROM SACCHAROMYCES CEREVISIAE
}

\author{
By \\ Emily Hopwood, B.Sc.H.
}

\begin{abstract}
A thesis submitted to the Faculty of Graduate and Postdoctoral Affairs in partial fulfilment of the requirements for the degree of

Master of Science

in
\end{abstract}

Biology

Department of Biology

Ottawa-Carleton Institute of Biology

Carleton University

Ottawa, Ontario

December 2012

(C)2012

Emily Hopwood 
Library and Archives

Canada

Published Heritage

Branch

395 Wellington Street

Ottawa ON K1A ON4

Canada
Bibliothèque et

Archives Canada

Direction du

Patrimoine de l'édition

395 , rue Wellington

Ottawa ON K1A ON4

Canada
Your file Votre référence

ISBN: 978-0-494-94293-2

Our file Notre référence

ISBN: $978-0-494-94293-2$
NOTICE:

The author has granted a nonexclusive license allowing Library and Archives Canada to reproduce, publish, archive, preserve, conserve, communicate to the public by telecommunication or on the Internet, loan, distrbute and sell theses worldwide, for commercial or noncommercial purposes, in microform, paper, electronic and/or any other formats.

The author retains copyright ownership and moral rights in this thesis. Neither the thesis nor substantial extracts from it may be printed or otherwise reproduced without the author's permission.
AVIS:

L'auteur a accordé une licence non exclusive permettant à la Bibliothèque et Archives Canada de reproduire, publier, archiver, sauvegarder, conserver, transmettre au public par télécommunication ou par l'Internet, prêter, distribuer et vendre des thèses partout dans le monde, à des fins commerciales ou autres, sur support microforme, papier, électronique et/ou autres formats.

L'auteur conserve la propriété du droit d'auteur et des droits moraux qui protege cette thèse. $\mathrm{Ni}$ la thèse ni des extraits substantiels de celle-ci ne doivent être imprimés ou autrement reproduits sans son autorisation.
In compliance with the Canadian Privacy Act some supporting forms may have been removed from this thesis.

While these forms may be included in the document page count, their removal does not represent any loss of content from the thesis.
Conformément à la loi canadienne sur la protection de la vie privée, quelques formulaires secondaires ont été enlevés de cette thèse.

Bien que ces formulaires aient inclus dans la pagination, il n'y aura aucun contenu manquant. 


\section{ABSTRACT}

Enzymes catalyzing transformations of amino acids rely on the catalytically versatile pyridoxal-5'-phosphate (PLP) cofactor. The specificity required for these reactions is provided by the protein scaffold through active-site residues that position the cofactor, substrate, and catalytic apparatus. The enzymes of the transsulfuration pathways interconvert cysteine and homocysteine, the direct precursor to methionine. Cystathionine $\gamma$-lyase (CGL) catalyzes the final step of the mammalian reverse transsulfuration pathway, hydrolyzing cystathionine to produce cysteine, and has gained interest due to its ability to also produce hydrogen sulfide, a neuromodulator which has a role in regulating hypertension by relaxing smooth muscle. While previous studies have elucidated the roles of active-site residues of the structurally similar enzymes of the bacterial transsulfuration pathway, which are potential targets for antibiotic agents, a detailed understanding of the CGL active site is lacking.

In this study, a set of 21 site-directed replacements of 10 active-site residues of yeast CGL (yCGL) were characterized to identify their roles in determining substrate and reaction specificity. Residues $\mathrm{R} 51, \mathrm{R} 108, \mathrm{~N} 232$ and $\mathrm{R} 369$ were found to interact with the substrate. Residue S52 does not have a significant role in substrate binding or catalysis. The catalytic lysine is tethered and guided by $\mathrm{Y} 49$ and $\mathrm{S} 334$, and Y103 is a unique determinant of $\gamma$-elimination reaction specificity. Residues E48 and E333 are responsible for maintaining charge distribution required for proper substrate binding and catalysis; in the case of E333, this affects the specificity of yCGL. These results further an understanding of the determinants of substrate and reaction specificity among PLPdependent enzymes, knowledge which will aid the design of antibiotics to selectively 
inhibit the bacterial transsulfuration enzymes and allow the development of antihypertensive therapeutics targeting CGL-catalyzed $\mathrm{H}_{2} \mathrm{~S}$ production in vascular tissue. 


\section{ACKNOWLEDGEMENTS}

First and foremost, I am immensely grateful for the guidance of my supervisor, Dr. Susan M. Aitken. Since my time as an undergraduate student, she has been a role model and mentor. She has shown continual patience with my learning process, challenged me to push myself to the limits of my learning, and given me countless opportunities to grow, both as a student of science and as a human being. Working in her laboratory has been an immense privilege, a formative experience, and an opportunity for which I am exceptionally thankful.

The critical insights and helpful feedback from my committee members, Dr. Iain Lambert and Dr. Amanda MacFarlane, were essential to creating this thesis, and to them I offer sincere thanks for their guidance throughout my studies.

Special thanks go to Pratik Lodha for training me in many laboratory techniques, and for making me laugh more times than I can remember. In learning from him I was able to become more independent and enthusiastic with my experimental work. The camaraderie I shared with Nikita Rayne, Nicholas Humphreys, and Adrienne Manders greatly enriched my time in the lab, and I will cherish the memories of working with them. I would also like to extend thanks to my fellow graduate students Edgar AbouAssaf, Dominique Morneau, and Allison Jaworski for always being willing to help when I encountered technical challenges.

The close friendships I am lucky to share with Pearl Cohen, Celeste Ali-Akow and Robin Magder have been integral to my ability to complete this degree. They have accepted me with my many flaws and been patient and understanding with me over and 
over again. Their emotional support throughout the years has been more valuable than words can describe.

This thesis would not have been possible without the constant encouragement and limitless love from my wonderful family. Even though separated by several time zones, my mother, Kimberley Hopwood, and sisters, Eleanor and Madeleine Hopwood, have always been there for me with unconditional support. The presence of my father, Dan Sharon, and my grandmother, Felicia Sharon, and the constant generosity they have shown me on a daily basis with their time and energy has provided me with strength to continue innumerable times when I was ready to give up. For believing in me even when I did not, and for maintaining my sanity in stressful times, I offer them all my humble gratitude.

Finally my grandfather, Hanoch Sharon, always pushed me to pursue my goals but passed away before I completed my graduate studies. This thesis is dedicated to his memory. 
TABLE OF CONTENTS

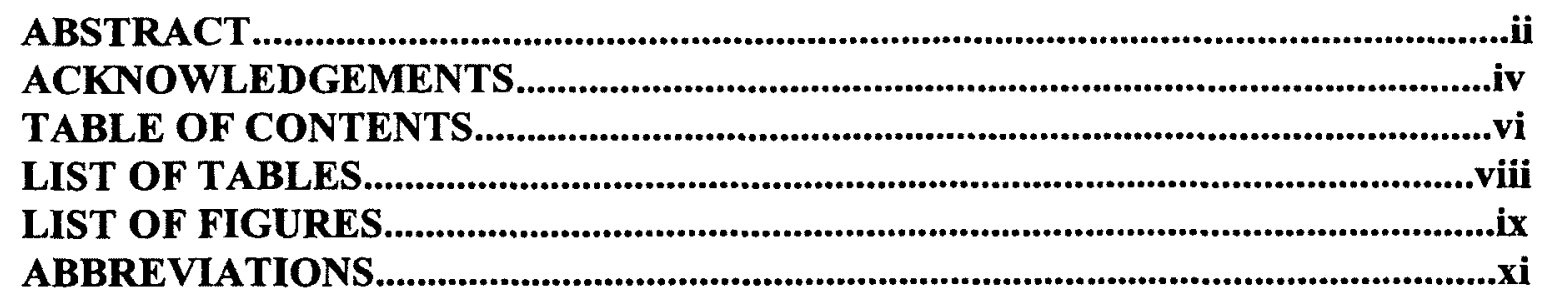

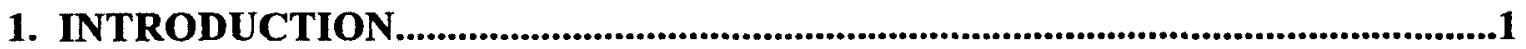

1.1. Metabolism of Sulfur-Containing Amino Acids......................................................

1.2. The Transsulfuration and Reverse Transsulfuration Pathways................................

1.3. PLP-Dependent Enzymes.............................................................................6

1.4. Cystathionine $\gamma$-Lyase................................................................................

1.5. Three-Dimensional Structure and Active-Site Architecture of yCGL.................12

1.6. Reaction Mechanism of yCGL..........................................................................25

1.7. Role of CGL in Cystathioninuria.......................................................................27

1.8. Role of CGL in Hydrogen Sulfide Production....................................................28

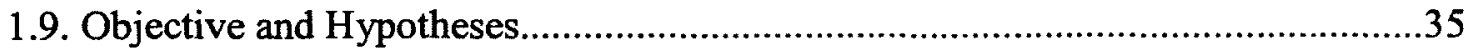

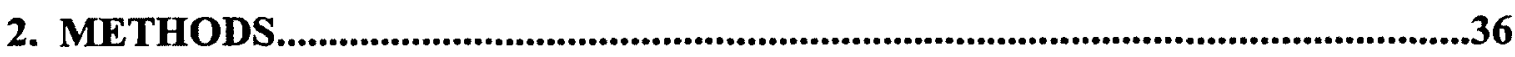

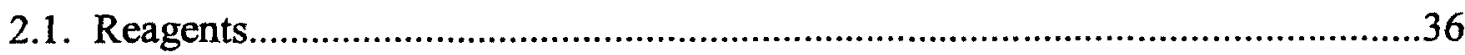

2.2. Construction, expression and purification of site-directed variants......................36

2.3. Determination of steady-state kinetic parameters for hydrolysis of L-Cth, L-Cys,

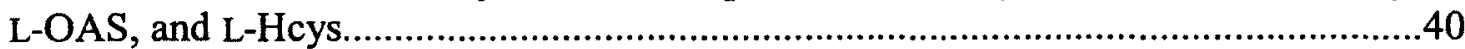

2.4. Evaluation of the $\mathrm{pH}$ dependence of wild-type $\mathrm{yCGL}$ and site-directed variants. 42

2.5. Absorbance Spectroscopy.........................................................................................43

2.6. Spectrophotometric Titration of Internal Aldimine...............................................43

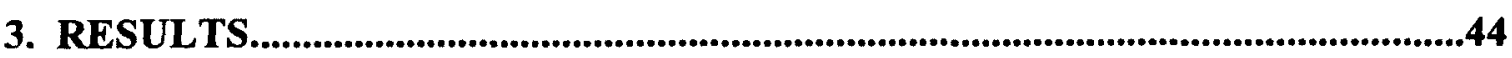

3.1. Construction, expression, and purification of the site-directed variants.................44

3.2. The wild-type yCGL enzyme...........................................................................4

3.3. The R51A/K, S52A/R, R108A/K, N232A, and R369A/K variants.....................57

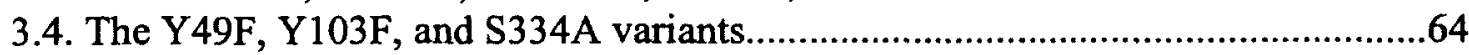

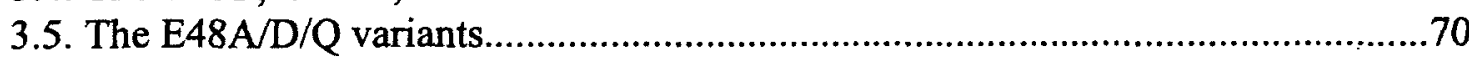

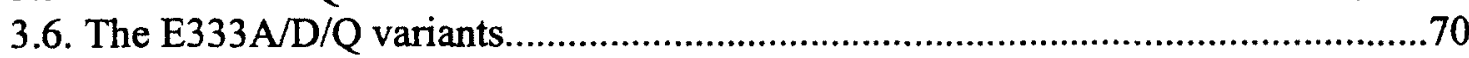

3.7. The E48/E333 alanine, aspartate, and glutamine double replacement variants.....75 


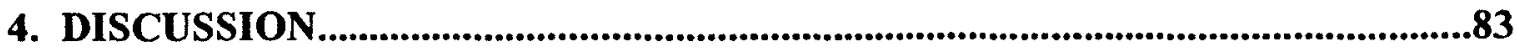

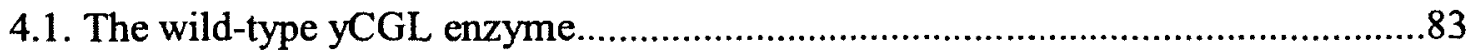

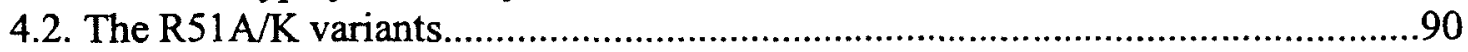

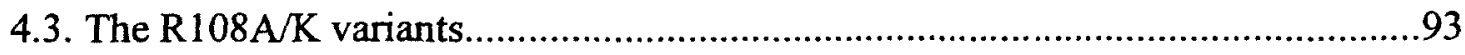

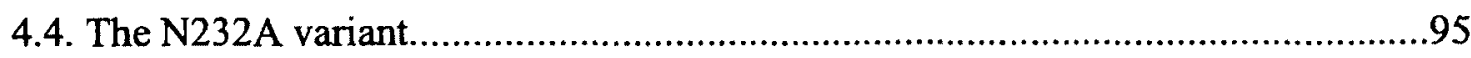

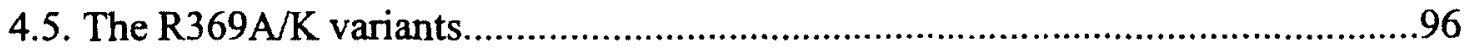

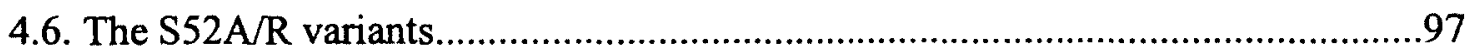

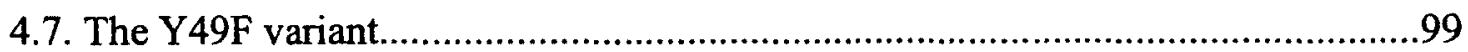

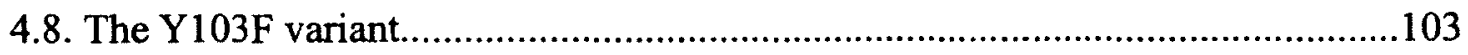

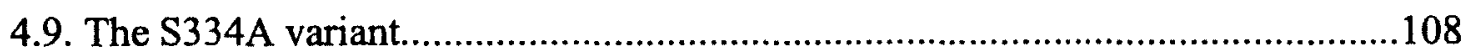

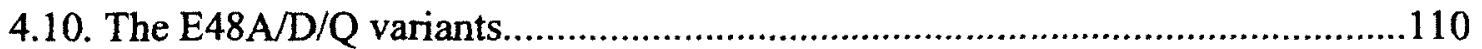

4.11. The E333A/D/Q variants....................................................................112

4.12. The E48/E333 alanine, aspartate, and glutamine double replacement variants.117

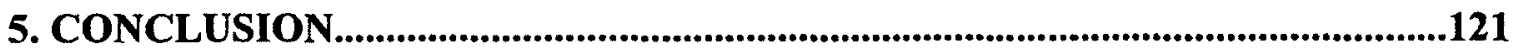

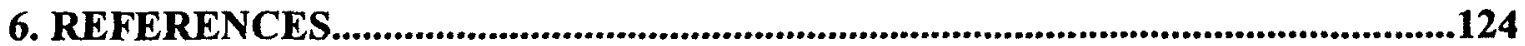




\section{LIST OF TABLES}

Table 2.1. Primers used in the construction of site-directed mutants of yCGL........37

Table 3.1. Yield of yCGL site-directed variants.............................................46

Table 3.2. Kinetic parameters for the hydrolysis of L-Cth, L-Cys, L-OAS, and L-Hcys

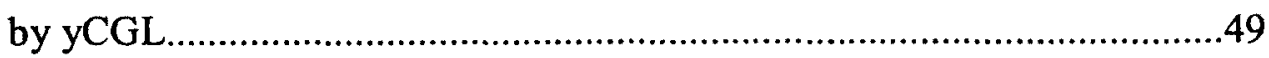

Table 3.3. Parameters determined from specific activity versus pH profiles of yCGL

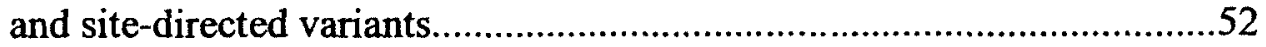

Table 3.4. Parameters determined from $k_{\text {cat }} / K_{\mathrm{m}}^{\mathrm{L}-\mathrm{Cth}}$ versus $\mathrm{pH}$ profiles of yCGL and site-directed variants....................................................................54

Table 3.5. Kinetic parameters of L-Cth, L-Cys, L-OAS and L-Hcys hydrolysis by yCGL and site-directed variants of residues R51, S52, R108, N232, and R369.

Table 3.6. Kinetic parameters of L-Cth, L-Cys, L-OAS and L-Hcys hydrolysis by yCGL and site-directed variants of residues Y49, Y103, and S334.

Table 3.7. Kinetic parameters of L-Cth, L-Cys, L-OAS and L-Hcys hydrolysis by yCGL and site-directed variants of residue E48.

Table 3.8. Kinetic parameters of L-Cth, L-Cys, L-OAS and L-Hcys hydrolysis by yCGL and site-directed variants of residue E333.

Table 3.9. Kinetic parameters of L-Cth, L-Cys, L-OAS and L-Hcys hydrolysis by yCGL and site-directed double replacement variants of residues E48/E333.

Table 4.1. Summary of effects of site-directed variants on kinetic parameters, $\mathrm{pH}$ dependence, and absorbance spectra of yCGL

Table 4.2. Comparison of roles of active-site residues in YCGL, eCBL, and eCGS..85 


\section{LIST OF FIGURES}

Figure 1.1. The bacterial/plant transsulfuration and mammalian/yeast reverse transsulfuration pathways which interconvert cysteine and homocysteine, the precursor to methionine. .4

Figure 1.2. Pyridoxal- 5 '-phosphate, the versatile cofactor involved in amino acid transformations. .7

Figure 1.3. Common mechanistic features of PLP-dependent enzymes catalyzing transformations of amino acids.

Figure 1.4. Cartoon representation of the yCGL homotetramer (PDB: 1N8P)...........13

Figure 1.5. Schematic representation of proposed contacts of L-Cth with active-site

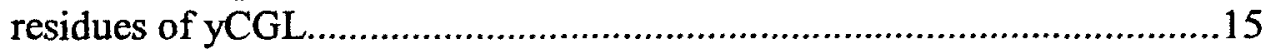

Figure 1.6. Targeted active-site residues of yCGL depicted in stick form..................16

Figure 1.7. Overlay of active-sites of yCGL (magenta; PDB 1N8P) and eCBL (blue; PCB 1CL1) showing residues E48 and E333 of yCGL and the corresponding F55 and Y338 of eCBL, proposed to be determinants of specificity.

Figure 1.8. Overlay of active-sites of yCGL (magenta; PDB 1N8P) and eCBL (blue; PCB 1CL1) showing conserved residues with putative roles in catalysis. 22

Figure 1.9. Proposed reaction mechanism of yCGL-catalyzed $\gamma$-elimination of LCth..

Figure 1.10. Schematic representation showing products arising from possible reactions catalyzed by yCGL on (A) the physiological L-Cth substrate or (B) the alternative substrates L-Cth, L-OAS, and L-Hcys.

Figure 1.11. Possible reactions of $\mathrm{L}$-alanine with a PLP-dependent enzyme. 33

Figure 3.1. Amplicons of the first and second rounds of overlap-extension PCR for construction of site-directed mutants.

Figure 3.2. SDS-PAGE demonstrating the purity of $5 \mu \mathrm{g}$ of wild-type and site-directed variants of yCGL.

Figure 3.3. The substrate and reaction specificity of wild-type yCGL......................50

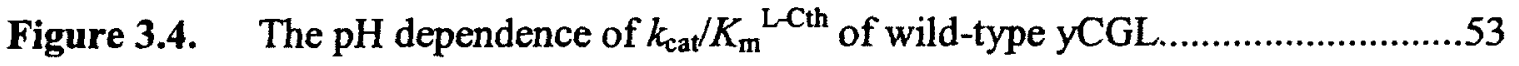

Figure 3.5. Spectrophotometric titration of (A) yCGL and (B) eCBL, showing dependence on $\mathrm{pH}$ of absorbance at $421 \mathrm{~nm}$ and $390 \mathrm{~nm}$. 
Figure 3.6. Absorbance spectrum of PLP cofactor of wild-type yCGL before and after incubation for 120 min with (A) $100 \mathrm{mM} \mathrm{L}$-alanine or (B) $3 \mathrm{mM} \mathrm{L-Cth....56}$

Figure 3.7. The $\mathrm{pH}$ dependence of specific activity of L-Cth hydrolysis of wild-type yCGL and the (A) R51A and (B) R51K variants. .59

Figure 3.8. The effect of 120 -min incubation with (A) $100 \mathrm{mM}$-alanine and (B) $3 \mathrm{mM}$ L-Cth on the absorbance spectra of the PLP cofactor of wild-type yCGL and the $\mathrm{R} 51 \mathrm{~A}$ and $\mathrm{R} 51 \mathrm{~K}$ variants. .60

Figure 3.9. The $\mathrm{pH}$ dependence of specific activity of L-Cth hydrolysis of wild-type yCGL (solid circle) and the R108A (empty circle) and R108K (triangle) variants.

Figure 3.10. The pH dependence of specific activity of L-Cth hydrolysis of wild-type yCGL and the S52A, S52R, and N232A site-directed variants.

Figure 3.11. Comparison of the $\mathrm{pH}$ dependence of specific activity of L-Cth hydrolysis for wild-type yCGL and (A) Y56F, (B) Y103F and (C) S334A. .66

Figure 3.12. The effect of 120 -min incubation with (A) $100 \mathrm{mM}$-alanine and (B) $3 \mathrm{mM}$ L-Cth on the absorbance spectra of the PLP cofactor of wild-type yCGL and the $\mathrm{Y} 49 \mathrm{~F}, \mathrm{Y} 103 \mathrm{~F}$ and S334A variants .67

Figure 3.13. Comparison of $\mathrm{pH}$ dependence of catalytic efficiency of L-Cth hydrolysis for wild-type yCGL and Y103F. .69

Figure 3.14. The $\mathrm{pH}$ dependence of the catalytic efficiency of L-Cth hydrolysis of wildtype yCGL and the E48A, E48D, and E48Q variants.

Figure 3.15. The effect of 120 -min incubation with (A) $100 \mathrm{mM} \mathrm{L}$-alanine and (B) $3 \mathrm{mM}$ L-Cth on the absorbance spectra of the PLP cofactor of wild-type yCGL and the E48A, E48D, and E48Q variants. .73

Figure 3.16. The $\mathrm{pH}$ dependence of the catalytic efficiency of L-Cth hydrolysis for wild-type yCGL and the (A) E333A, (B) E333D, and (C) E333Q variants. . .76

Figure 3.17. The effect of 120 -min incubation with (A) $100 \mathrm{mM}$ L-alanine and (B) $3 \mathrm{mM}$ L-Cth on the absorbance spectra of the PLP cofactor of wild-type yCGL and the E333A, E333D and E333Q variants. .77

Figure 3.18. Comparison of the $\mathrm{pH}$ dependence of the catalytic efficiency of L-Cth hydrolysis for wild-type yCGL and the (A) E48A/E333A, (B) $\mathrm{E} 48 \mathrm{D} / \mathrm{E} 333 \mathrm{D}$, and $(\mathrm{C}) \mathrm{E} 48 \mathrm{Q} / \mathrm{E} 333 \mathrm{Q}$ variants .80

Figure 3.19. The effect of 120 -min incubation with (A) $100 \mathrm{mM} \mathrm{L}$-alanine and (B) $3 \mathrm{mM}$ L-Cth on the absorbance spectra of the PLP cofactor of wild-type yCGL and the $\mathrm{E} 48 \mathrm{~A} / \mathrm{E} 333 \mathrm{~A}, \mathrm{E} 48 \mathrm{D} / \mathrm{E} 333 \mathrm{D}$ and $\mathrm{E} 48 \mathrm{Q} / \mathrm{E} 333 \mathrm{Q}$ variants. 


\section{ABBREVIATIONS}

AAT Aspartate aminotransferase

ALAS 5-aminolevulinate synthase

AMPSO

3-[1,1-dimethyl-2-hydroxyethyl)amino]-2-hydroxypropanesulfonic

acid

ATP

Adenosine triphosphate

AVG Aminoethoxyvinylglycine

CAPS N-cyclohexyl-3-aminopropanesulfonic acid

CBL

Cystathionine $\beta$-lyase

CBS

Cystathionine $\beta$-synthase

CGL

Cystathionine $\gamma$-lyase

CGS

Cystathionine $\gamma$-synthase

$\mathrm{CO}$

Carbon monoxide

DNA

Deoxyribonucleic acid

DTNB

5,5'-Dithiobis-(2-nitrobenzoic acid)

E. coli

Escherichia coli

E.C.

Enzyme Commission

eCBL

Escherichia coli cystathionine $\beta$-lyase 
eCGS

GSH

$\mathrm{H}_{2} \mathrm{~S}$

hCBS

hCGL

HO-HxoDH

IPTG

L-Cth

L-Cys

L-Hcys

LDH

L-OAS

L-OSHS

MGL

MIM

MOPS

mRNA
Escherichia coli cystathionine $\gamma$-synthase

Glutathione

Hydrogen sulfide

Human cystathionine $\beta$-synthase

Human cystathionine $\gamma$-lyase

D-2-Hydroxyisocaproate dehydrogenase

Isopropyl- $\beta$ - $D$-thiogalactopyranoside

L-Cystathionine

L-Cysteine

L-Homocysteine

L-lactate dehydrogenase

$O$-acetyl-L-serine

$\mathrm{O}$-succinyl-L-homoserine

Methionine $\gamma$-lyase

Mendelian Inheritance in Man

3-(N-morpholino)propanesulfonic acid

Messenger RNA 
NADH

Ni-NTA

NO

OASS

OE-PCR

PCR

PDB

PLP

PMP

RNA

S. cerevisiae

SAM

SAT

SDS-PAGE

TAPS

THF

yCGL
Nicotinamide adenine dinucleotide, reduced form

Nickel-nitrilo triacetic acid

Nitric oxide

$O$-acetyl- L-serine sulfhydrylase

Overlap-extension polymerase chain reaction

Polymerase chain reaction

Protein Data Bank

Pyridoxal 5'-phosphate

Pyridoxamine 5'-phosphate

Ribonucleic acid

Saccharomyces cerevisiae

$S$-adenosyl-methionine

Serine acetyltransferase

Sodium dodecyl sulfate polyacrylamide gel electrophoresis

N-Tris(hydroxymethyl)methyl-3-aminopropanesulfonic acid

Tetrahydrofolate

Yeast cystathionine $\gamma$-lyase 


\section{INTRODUCTION}

\subsection{Metabolism of Sulfur-Containing Amino Acids.}

Sulfur is a biologically important element, forming a component of many molecules such as amino acids, proteins, and vitamins (Atmaca, 2004). Methionine and cysteine, the two proteinogenic, sulfur-containing amino acids, have important roles in cellular metabolism. Methionine is hydrophobic, found usually in the core of globular proteins or in the domains of membrane-spanning proteins which interact with the lipid bilayer, and is the initiating amino acid in eukaryotic protein synthesis (Brosnan and Brosnan, 2006). The side chain of cysteine has a free thiol group that can form disulfide bridges with other cysteine residues to maintain protein structure and ensure proper protein folding (Brosnan and Brosnan, 2006).

Methionine lies at the branchpoint of several metabolic pathways. It is the precursor of $S$-adenosylmethionine (SAM), the ubiquitous and versatile methyl donor that plays a central role in cellular metabolism (Finkelstein and Martin, 2000). In addition to directly modifying proteins, DNA, RNA, and other small molecules by methylation, SAM also functions as a metabolic effector by acting as an allosteric regulator of enzyme activity (Finkelstein and Martin, 2000; Brosnan and Brosnan, 2006).

Cysteine (L-Cys) is used in protein synthesis, and is the cellular precursor to coenzyme A, sulfate, and hydrogen sulfide (Dominy and Stipanuk, 2004). Additionally, L-Cys is a component of the tripeptide glutathione (GSH), which is synthesized in a twostep process comprising the addition of glutamate and glycine (Wu et al., 2004). Glutathione regulates intracellular redox status and prevents oxidation of biomolecules by 
both directly and indirectly scavenging free radicals and other reactive oxygen species (Wu et al., 2004). Glutathione is also involved in detoxification of xenobiotics, and as a substrate for peroxidases and GSH-transferases (Atmaca, 2004).

\subsection{The Transsulfuration and Reverse Transsulfuration Pathways.}

Plants can reduce inorganic sulfate to hydrogen sulfide to synthesize sulfurcontaining amino acids de novo, but animals cannot (Atmaca, 2004). In mammals, the requirement for methionine is absolute, but L-Cys can be synthesized from methionine (Dominy and Stipanuk, 2004). Homocysteine (L-Hcys), the immediate precursor of methionine, and L-Cys are interconverted by the transsulfuration pathways (Figure 1.1). In plants and bacteria L-Cys is converted to L-Hcys through the transsulfuration pathway. In contrast, mammals and yeast synthesize L-Cys from L-Hcys via the reverse transsulfuration pathway (Thomas and Surdin-Kerjan, 1997; Steegborn et al., 1999). Since the transsulfuration pathway is found in plants and bacteria but not in mammals, the enzymes of this pathway are attractive targets for antibiotic or herbicidal agents.

Condensation of L-Cys with a homoserine ester forms L-cystathionine (L-Cth) in the first step of the transsulfuration pathway (Figure 1.1; Clausen et al., 1998). This $\gamma$ replacement reaction is catalyzed by cystathionine $\gamma$-synthase (CGS; EC 2.5.1.48), encoded by the metB gene in Escherichia coli (Clausen et al., 1998). The homoserine ester used is species-specific: $O$-succinylhomoserine (L-OSHS), $O$-acetylhomoserine, and $O$-phosphohomoserine are employed by bacterial, fungal, and plant CGS enzymes, respectively (Yamagata 1989; Cherest et al., 1993; Datko et al., 1974). Cystathionine is 
subsequently hydrolyzed to yield L-Hcys, pyruvate and ammonia, in the second step of the transsulfuration pathway (Figure 1.1; Clausen et al., 1996). This $\beta$-elimination reaction is catalyzed by the enzyme cystathionine $\beta$-lyase (EC 4.4 .1 .8 ), encoded by the metC gene in E. coli (Clausen et al., 1996).

Several intracellular pathways involve L-Hcys (Finkelstein, 1998). In addition to feeding into the mammalian reverse transsulfuration pathway, L-Hcys can be converted to methionine by the action of the enzyme methionine synthase, which uses the methyl donor 5-methyltetrahydrofolate to add a methyl group onto the free thiol of L-Hcys, releasing tetrahydrofolate (THF). Methionine can then be adenosylated to make SAM, and THF can be recycled back to 5-methylTHF (Finkelstein, 1998).

Mammals and some fungi, including Saccharomyces cerevisiae, synthesize L-Cys from the essential amino acid methionine via the reverse transsulfuration pathway (Cooper, 1983). A $\beta$-replacement reaction, in which L-Hcys and serine are condensed to form L-Cth, comprises the first step of the reverse transsulfuration pathway (Figure 1.1; Thomas and Surdin-Kerjan, 1997). This step is catalyzed by the enzyme cystathionine $\beta$ synthase (CBS; EC 4.2.1.22), encoded by the CYS4 gene in S. cerevisiae (Jhee et al., 2000). A $\gamma$-elimination reaction, in which the L-Cth intermediate is hydrolyzed to produce L-Cys, $a$-ketobutyrate and ammonia, is the second and final step of the reverse transsulfuration pathway (Figure 1.1) and is catalyzed by cystathionine $\gamma$-lyase (CGL; EC 4.4.1.1), encoded by the CYS3 gene in yeast (Yamagata et al., 1993). 

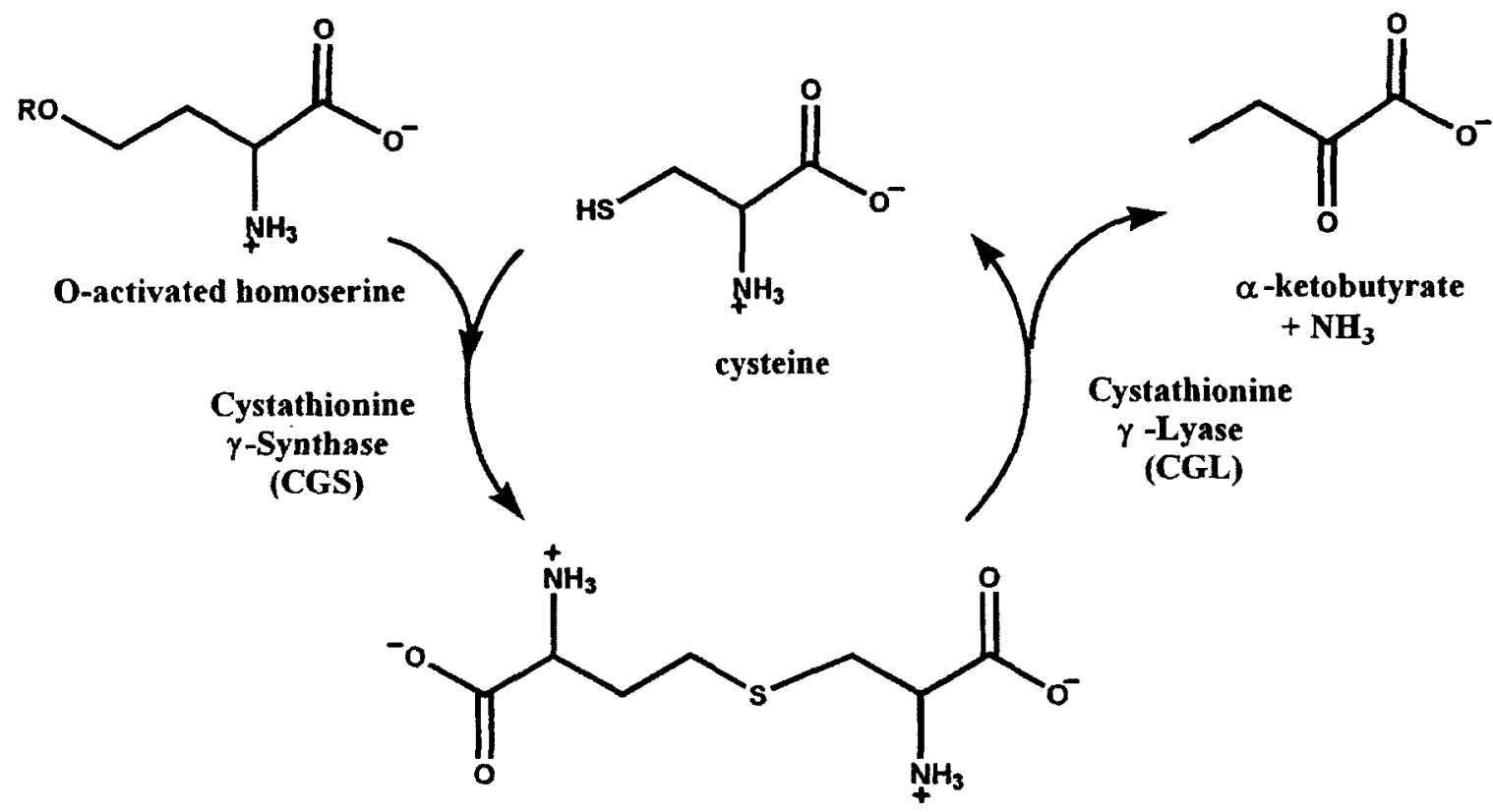<smiles>CC(=O)C(=O)[O-]</smiles>
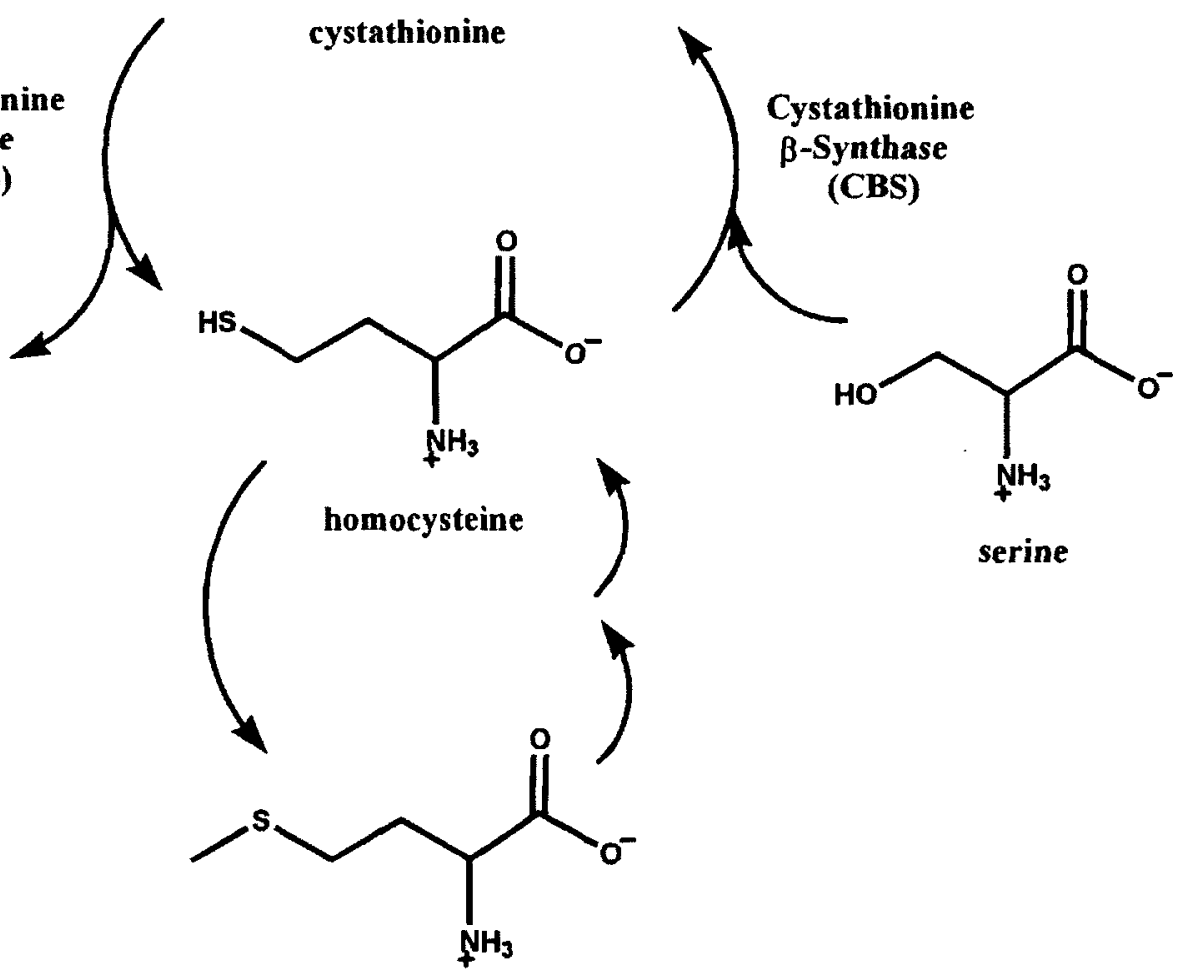

methionine

Figure 1.1. The bacterial/plant transsulfuration and mammalian/yeast reverse transsulfuration pathways which interconvert cysteine and homocysteine, the precursor to methionine. Cystathionine is the common intermediate of both pathways. Adapted from Steegborn et al. (1999). 
Yeast were also proposed to have the ability to synthesize L-Cys through a nontranssulfuration pathway, involving the serine acetyltransferase (SAT)-catalyzed formation of $O$-acetyl-L-serine (L-OAS) which can be directly sulfhydrylated by the enzyme $O$-acetyl-L-serine sulfhydrylase (OASS) to produce L-Cys (Ono et al., 1999). However, the SAT/OASS pathway likely does not comprise a physiologically significant L-Cys biosynthetic pathway in yeast (Ono et al., 1999), such that L-Cys is produced primarily via the reverse transsulfuration pathway (Takagi et al., 2003). Thus the CBScatalyzed reaction is the committed step in L-Cys synthesis (Finkelstein, 1998). The flux between the conversion of L-Hcys to L-Cys, via transsulfuration, and the remethylation of L-Hcys to produce methionine, is modulated by SAM, which acts as an activator of CBS, thereby increasing the flux through the transsulfuration pathway (Finkelstein, 1998).

The enzymes of the reverse transsulfuration pathway also affect the redox state of the cell. The L-Cys produced by CGL is a precursor of GSH, which in turn plays a major role in maintaining intracellular redox status (Diwakar and Ravindranath, 2007), as demonstrated by the decrease in GSH levels and increased oxidative damage to mitochondria which accompany a decrease in CGL activity (Diwakar and Ravindranath, 2007). Another level of redox regulation may exist, due to the presence of a heme group in the human form of the CBS enzyme (Kery et al., 1994). Since L-Cys is the limiting reagent in production of GSH (Diwakar and Ravindranath, 2007), the ability to modulate CBS activity in response to the redox potential would allow the cell to respond to oxidative stress. The function of the heme ligand of human CBS (hCBS) is still unclear, but the observation that hCBS is approximately 2 -fold more active when the heme is in its oxidized form has led to the suggestion that CBS can act as a redox sensor (Taoka et 
al., 1998). However, the relationship between CBS activity and the redox state of the heme is influenced by other factors such as $\mathrm{pH}$ (Pazicni et al., 2004), and a simple mechanism of redox regulation of CBS appears unlikely.

\subsection{PLP-Dependent Enzymes.}

The enzymes of the transsulfuration pathways are all dependent on the pyridoxal5'-phosphate (PLP) cofactor (Figure 1.2), a derivative of vitamin $\mathrm{B}_{6}$ (Christen and Mehta, 2001). Enzymes that employ the versatile PLP cofactor are found in all organisms. The catalytic versatility of this cofactor is demonstrated by the observation that PLPdependent enzymes are found in 5 of the 6 classes defined by the Enzyme Commission (Christen and Mehta, 2001). PLP-mediated transformations of amino acids include sidechain replacement or elimination, $a$-decarboxylation, $a$-amino group transfer such as transamination, and racemization, the interconversion of $L-$ and $D$-forms (Percudani and Peracchi, 2003). The versatility of PLP as a cofactor arises from its ability to act as an electrophilic catalyst (Percudani and Peracchi, 2003). The extended $\pi$-electron system of the pyridinium ring of the PLP cofactor (Figure 1.2) acts as an electron sink, "storing" electrons from broken bonds through electron delocalization, and stabilizing the developing negative charge as different reactive carbanionic intermediates are formed, thereby enabling the observed diversity of transformations of amino acid substrates (Bach et al., 1999; Christen and Mehta, 2001). It is the protein component of a given PLPdependent enzyme that enforces substrate and reaction specificity. 


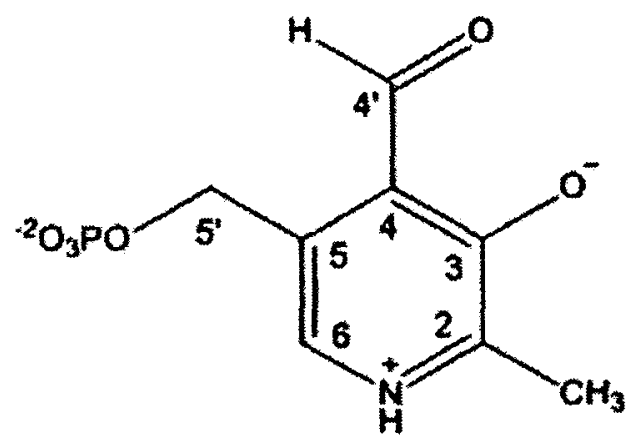

Figure 1.2. Pyridoxal-5'-phosphate, the versatile cofactor involved in amino acid transformations. 
Certain common mechanistic features are shared by all PLP-dependent enzymes catalyzing transformations of amino acids (Figure 1.3). The aldehyde group of the PLP cofactor is covalently bound by a Schiff base linkage to the $\varepsilon$-amino group of an activesite lysine residue (Christen and Mehta, 2001; Schneider et al., 2000; Jansonius, 1998). The $\alpha$-amino group of the incoming substrate replaces that of the active-site lysine, via a transaldimination reaction, forming the external aldimine (Figure 1.3, reaction 1) (Hayashi 1995; Christen and Mehta, 2001). This step is common to all PLP-dependent enzymes. When the substrate and cofactor are linked together, the $C_{\alpha} \sigma$-bond, of the amino acid substrate, positioned orthogonal to the plane of the cofactor's $\pi$-system is weakened, thereby facilitating its cleavage (Dunathan, 1966). It is the bond perpendicular to the plane of the pyridoxal ring that is specifically weakened because delocalization with the $\pi$-orbital system of the cofactor decreases its electron density (Dunathan, 1966). The carbanion resulting from cleavage of one of the $C_{\alpha}$ bonds is delocalized and stabilized by the pyridinium ring of the cofactor (Bach et al., 1999; Schneider et al., 2000). Therefore, depending on the orientation of the hydrogen, carboxylate and sidechain groups attached to $C_{a}$ of the substrate, distinct reaction pathways will be followed. Abstraction of the $\alpha$-proton (Figure 1.3, reaction 2) is the common first step shared by transamination, racemization, and $\alpha / \beta$ or $\alpha / \gamma$ side chain rearrangement (Jansonius, 1998). 


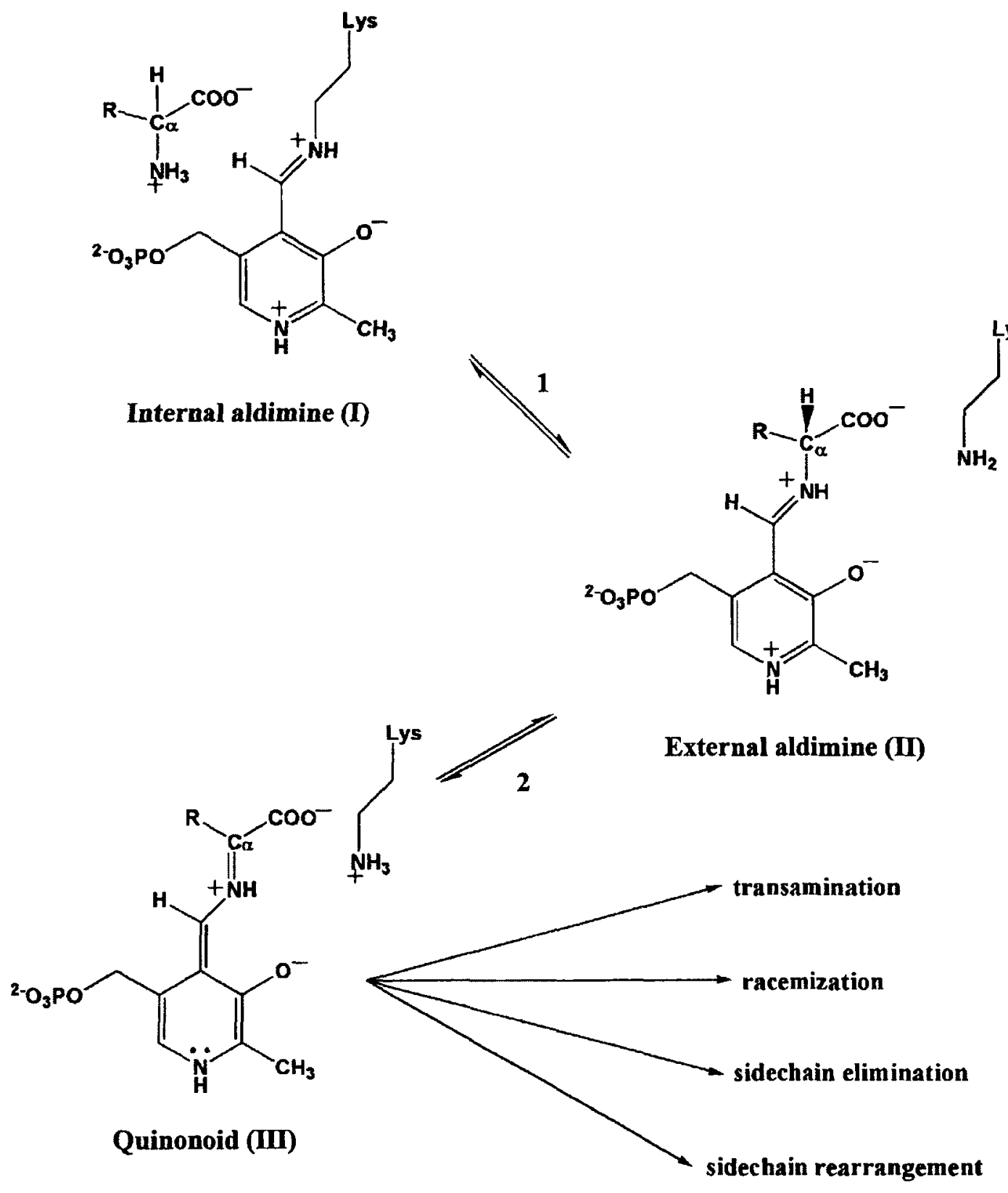

Figure 1.3. Common mechanistic features of PLP-dependent enzymes catalyzing transformations of amino acids. Formation of an external aldimine linkage between PLP and the amino acid substrate occurs via transaldimination with the catalytic lysine (reaction 1), and $\alpha$-proton abstraction (reaction 2) creates a quinonoid intermediate which can lead to a diversity of possible reactions (Jansonius, 1998; Schneider et al., 2000). 
The PLP-dependent enzymes catalyzing transformations of amino acid substrates are classified into four structural families, or fold types, based on tertiary structure and active-site construction. Fold type I includes aminotransferases, decarboxylases, and $\alpha$-, $\beta-$, or $\gamma$-eliminases and is exemplified by the extensively studied model PLP-dependent enzyme aspartate aminotransferase (AAT) (Jansonius 1998; Percudani and Peracchi, 2003). Both enzymes of the transsulfuration pathway, CGS and CBL, as well as CGL, of the reverse transsulfuration pathway, are members of fold type I (Schneider et al., 2000). A characteristic of fold type I enzymes is the location of the active site at the interface between the subunits comprising the catalytic dimer. Although some fold type I enzymes, such as AAT, are homodimeric, others are higher order oligomers, as exemplified by the homotetrameric CGS, CBL and CGL. Each subunit possesses a large and a small domain. The lysine residue to which the PLP cofactor is bound is located in the larger domain and the phosphate group of the PLP cofactor is anchored to an N-terminal loop that immediately precedes a helix on the opposite side of the active site cleft. Residues from both monomers of the homodimer contribute to the formation of the active site (Schneider et al., 2000).

Fold type II includes enzymes that catalyze $\beta$-elimination reactions (Percudani and Peracchi, 2003), such as CBS of the reverse transsulfuration pathway (Jhee et al., 2000). Fold type II enzymes often possess a regulatory domain as well as the PLPbinding, catalytic domain. In contrast with fold type I enzymes, the active site is formed by residues of a single monomer (Schneider et al., 2000). Fold type III is characterized by an $\alpha / \beta$ barrel structure (Schneider et al., 2000). Alanine racemase and some decarboxylases fall into this fold type (Percudani and Peracchi, 2003). In fold type IV 
enzymes, similarly to fold type I, the active site is formed at the interface of two domains. However, PLP is bound with the re face of the pyridoxal ring, thereby accounting for difference in stereochemistry, D vs L, of its substrates (Schneider et al., 2000). D-alanine aminotransferase is representative of this fold type (Percudani and Peracchi, 2003).

\subsection{Cystathionine $\gamma$ - Lyase.}

Cystathionine $\gamma$-lyase (CGL) catalyzes the hydrolysis of $\mathrm{L}-\mathrm{C}$ th to produce $\mathrm{L}-\mathrm{Cys}$, $\alpha$-ketobutyrate, and ammonia. The $S$. cerevisiae CYS3 gene product, first characterized by Yamagata et al. (1993), was shown to have both cystathionine $\beta$-lyase and $\gamma$-lyase activity, but the 2 -fold greater $K_{\mathrm{m}}$ of the $\beta$-elimination reaction compared to the $\gamma$ elimination of L-Cth suggested a slight preference for cleavage of the $\mathrm{C}_{\gamma}-\mathrm{S}$ bond (Yamagata et al., 1993). However, the assays used by Yamagata et al. (1993) to distinguish $\beta$-versus $\gamma$-elimination of the $S$. cerevisae (yeast) CGL (yCGL) enzyme lack a high degree of specificity, and more reliable characterization of the reaction specificity of yCGL enzyme is required. Human CGL (hCGL) may have higher specificity for $\mathrm{C}_{\gamma}-\mathrm{S}$ bonds, as the rate of production of sulfhydryl groups arising from L-Cth hydrolysis are reported to match the rate of production of L-Cys, indicating that hCGL cleaves the L-Cth substrate almost exclusively at the $\mathrm{C}_{\gamma}-\mathrm{S}$ bond (Steegborn et al., 1999).

The yCGL enzyme shares $30-38 \%$ amino acid sequence identity with the $E$. coli CGS (eCGS) and CBL (eCBL) enzymes of the transsulfuration pathway, which are also members of the $\gamma$-subfamily of fold type I enzymes (Farsi et al., 2009). The $\gamma$-subfamily of the large and diverse fold type I comprises several closely related enzyme catalyzing 
side-chain rearrangements of sulfur-containing amino acids. The CYS3 gene product has the ability to complement $E$. coli metB and metC knockout strains which lack eCGS and eCBL activity, respectively, demonstrating that, in addition to low substrate specificity, the yeast CGL enzyme has low reaction specificity, as complementation of these strains requires the ability to carry out both $\gamma$-replacement and $\beta$-elimination reactions (Farsi et al., 2009). However, the catalytic efficiency of yCGL is two orders of magnitude lower than those of CGS and CBL of the transsulfuration pathway, largely due to a $k_{\text {cat }} \sim 10$-fold and 50-fold lower than those of CGS and CBL, respectively (Farsi et al., 2009).

The active sites of CGS, CBL, and CGL share a high degree of structural similarity, as indicated by the r.m.s. deviation of $\sim 1.5 \AA$ between 350 superimposed $C_{\alpha}$ atoms of their backbones (Messerschmidt et al., 2003). Determining the position, identity, and flexibility of key residues responsible for maintaining substrate and reaction specificity will be essential for ensuring that antibiotics or herbicides designed to selectively target bacterial or plant transsulfuration enzymes (CGS or CBL) will not inhibit the structurally similar human CGL enzyme (Lodha et al., 2010; Lodha and Aitken, 2011).

\subsection{Three-Dimensional Structure and Active-Site Architecture of $y C G L$.}

The crystal structure of yCGL has been solved, and indicates that this enzyme, like others of the $\gamma$-subfamily of fold type I, including CGS and CBL, is a homotetramer composed of a pair of active dimers (Figure 1.4). Each monomer of yCGL comprises 393 amino acids, and binds one PLP molecule (Messerschmidt et al., 2003). The active site of 


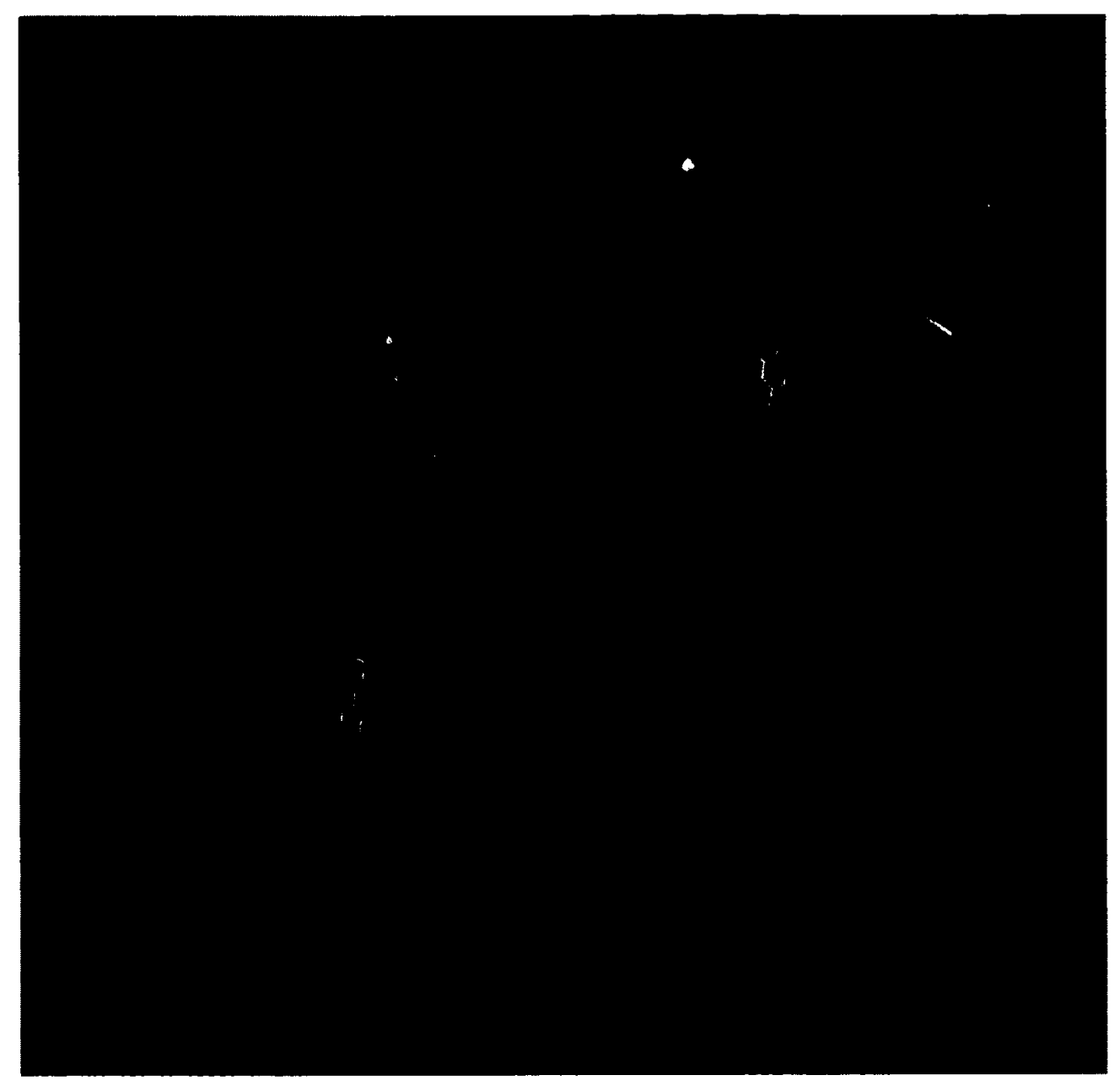

Figure 1.4. Cartoon representation of the yCGL homotetramer (PDB: 1N8P). The PLP cofactor (yellow) is shown in stick form (Messerschmidt et al., 2003). 
yCGL is situated at the interface between the PLP-binding domain and the C-terminal domain and includes several residues from the $\mathrm{N}$-terminal loop of the neighbouring monomer. The N-terminal domain (residues 1-54), which interacts with the neighbouring monomer's active site, comprises one helix, one $\beta$-strand, and an extended loop. The PLP-binding domain (residues 54-252) contains some of the residues necessary for catalysis, with the cofactor bound covalently to K203 (Messerschmidt et al., 2003).

The PLP cofactor is held in place in the active site by several interactions, in addition to the covalent bond to K203 (Figure 1.5). The phosphate group of PLP is tethered by hydrogen bonds to active-site residues including Y49 and R51. The pyridine ring of PLP is stabilized on the protein-facing side by T180 and S200, and is sandwiched on the other side by Y103 (Figure 1.6), via a $\pi$-stacking interaction. Roles for K203 and Y103 as catalytic residues have been proposed (Messerschmidt et al., 2003). The protonated aldimine, as well as the positively charged guanidinium group of the R51 side chain, are proposed to facilitate the ability of $\mathrm{Y} 103$ to participate in catalysis, by lowering the $\mathrm{p} K_{\mathrm{a}}$ of its hydroxyl group (Messerschmidt et al., 2003).

Based on the crystal structure of yCGL, a number of active-site residues (Figure 1.5) have been proposed to have key roles in the function of this enzyme, including substrate binding and catalysis. These active-site residues are conserved in the human CGL enzyme, which shares $50 \%$ amino acid identity and $67 \%$ amino acid similarity with 


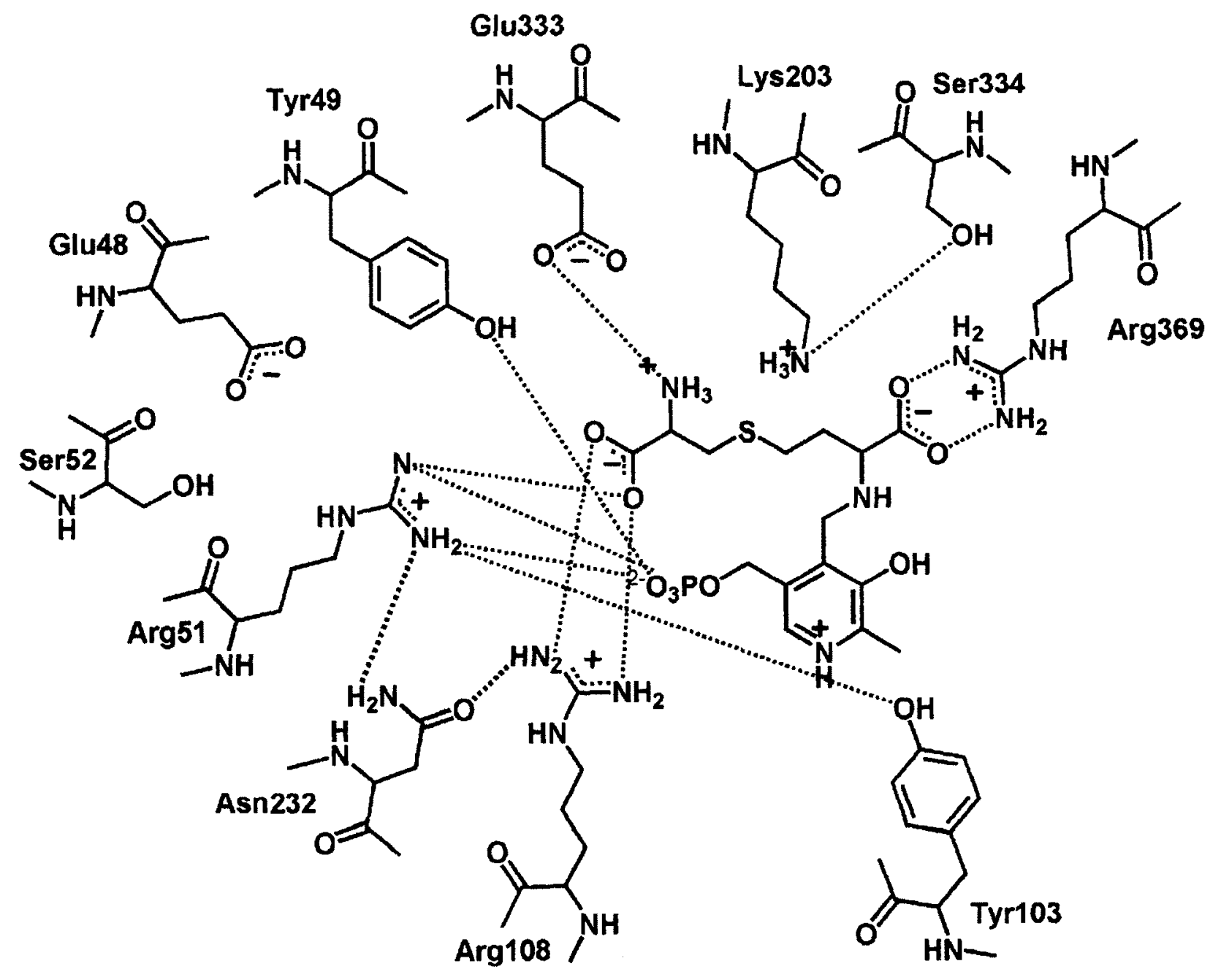

Figure 1.5. Schematic representation of proposed contacts of L-Cth with active-site residues of yCGL. Dotted lines indicate putative hydrogen-bonding interactions between heteroatoms separated by $<3.5 \AA$ (Messerschmidt et al., 2003) 


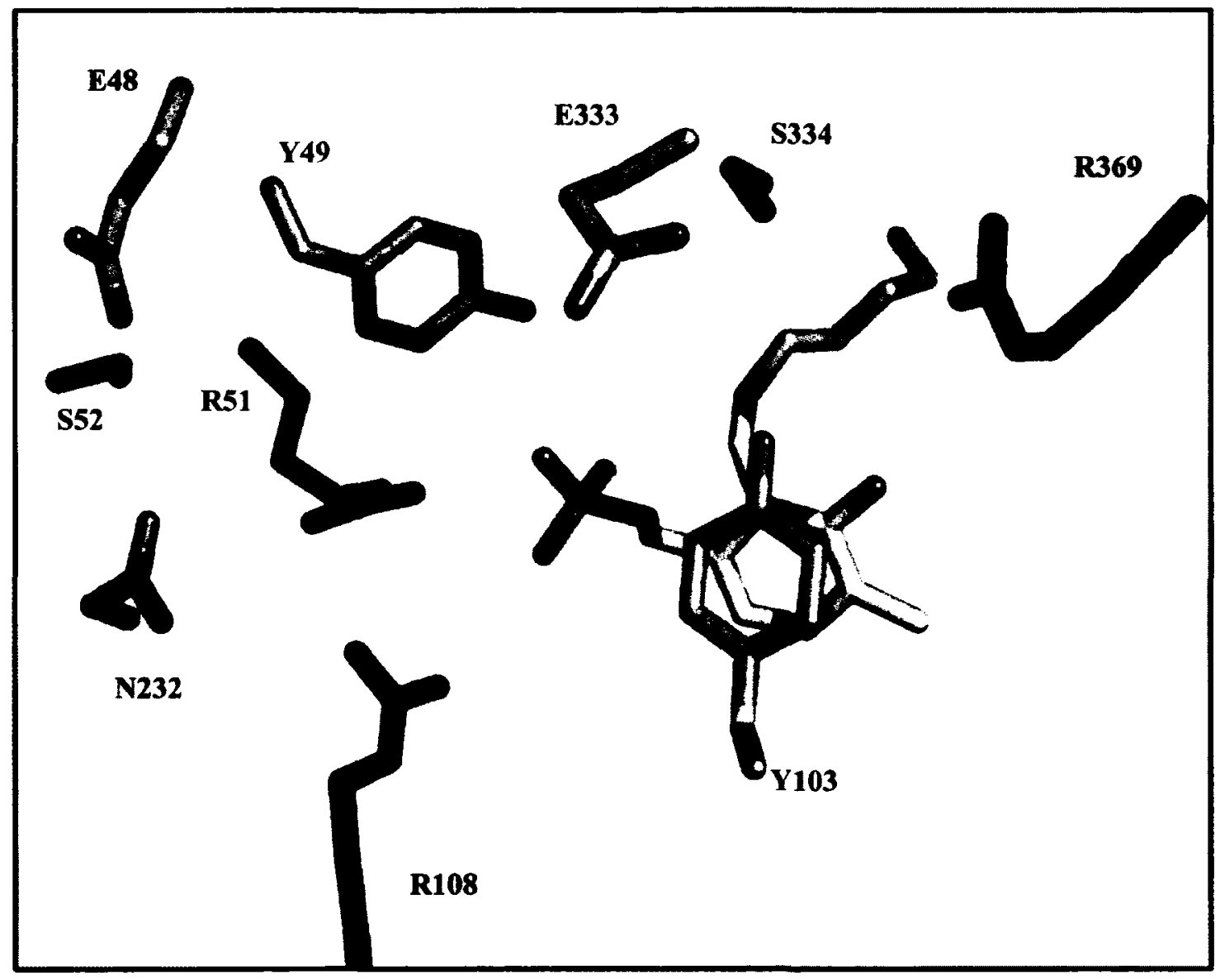

Figure 1.6. Targeted active-site residues of yCGL depicted in stick form. The PLP cofactor (yellow) is shown in covalent linkage to K203 (grey) (PDB: 1N8P) (Messerschmidt et al., 2003). 
yCGL (Steegborn et al., 1999). Site-directed mutagenesis studies have elucidated the roles of the corresponding residues in the active sites of eCGS and eCBL (Farsi et al., 2009; Lodha et al., 2010; Lodha and Aitken, 2011; Jaworski et al., 2012).

The CGL and CBL enzymes both bind the pseudosymmetric L-Cth substrate, but in different orientations, so differences in active-site architecture and the positioning of key residues may be important determinants of reaction specificity. Residues E48 and E333 of yCGL are conserved as acidic residues in eCGS (D45 and E325) but are replaced by aromatic residues in eCBL (F55 and Y338; Figure 1.7), and have therefore been proposed as determinants of reaction specificity (Messerschmidt et al., 2003). This reaction specificity was speculated to be enforced by electrostatic repulsion created between the carboxylate side chain of yCGL-E333 and the sulfur atom of L-Cth, thus favouring substrate binding in only the orientation appropriate for $\gamma$-elimination (Messerschmidt et al., 2003). Similarly, the corresponding tyrosine residue at this position, unique to bacterial CBL sequences, was expected to determine the orientation of L-Cth binding in this enzyme in a manner that would allow only $\beta$-elimination to occur (Clausen et al., 1998). However, the kinetic parameters of the eCBL-Y338F phenylalanine substitution variant for L-Cth, L-OAS and L-Cys hydrolysis are all within 2-fold of the wild type, indicating that the hydroxyl moiety of this residue is not a determinant of reaction specificity (Lodha and Aitken, 2011). A series of site-directed variants of yCGL (E48A,D,F and E333A,Y), designed to interconvert these conserved acidic residues with the corresponding aromatic residues of $\mathrm{eCBL}$, were investigated for their ability to complement $\triangle m e t B$ and $\triangle m e t C$ strains of $E$. coli lacking CGS and CBL activity, respectively (Farsi et al., 2009). While the catalytic efficiency of the substitution 


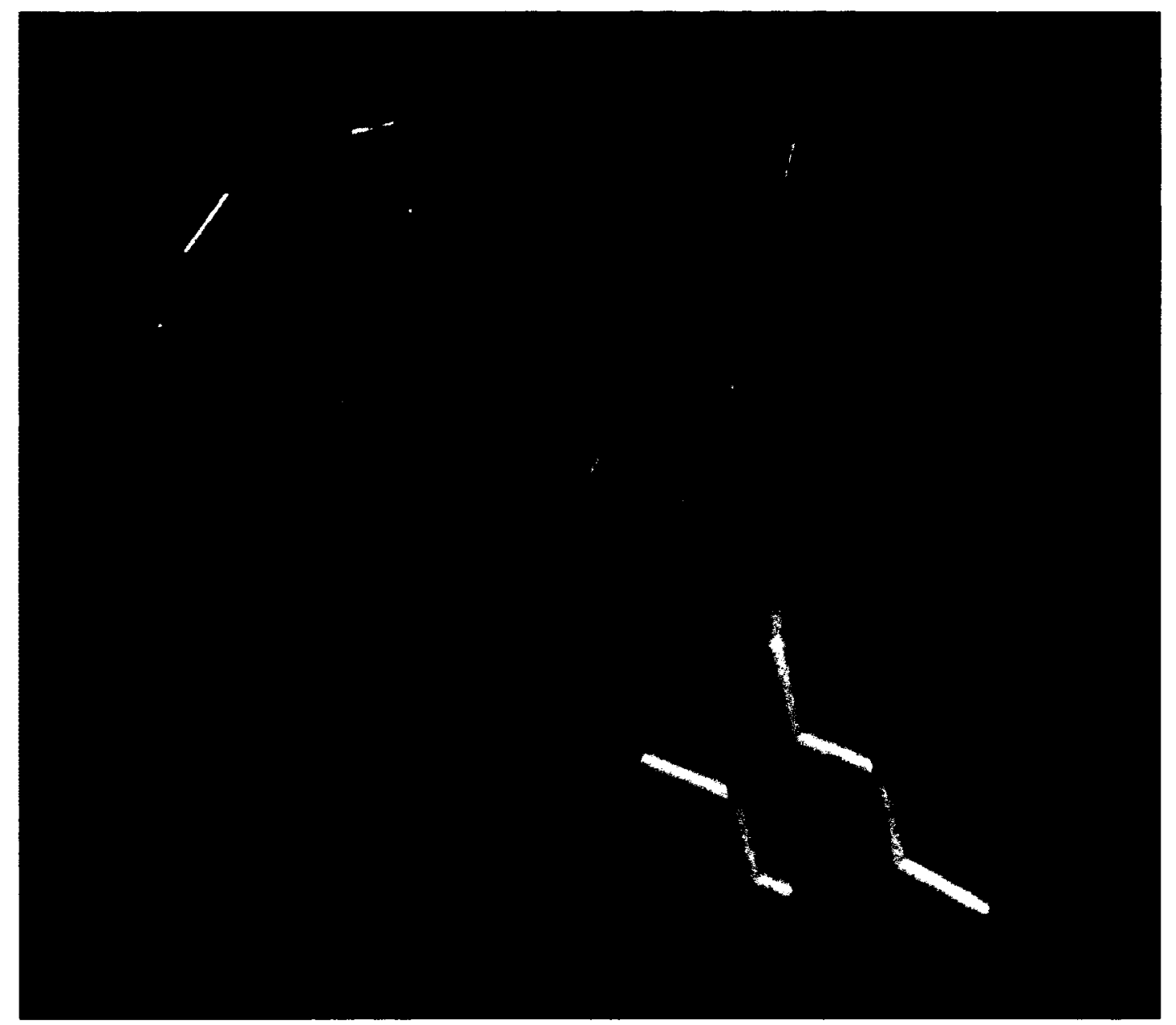

Figure 1.7. Overlay of active-sites of yCGL (magenta; PDB 1N8P) and eCBL (blue; PCB $1 C L 1)$ showing residues E48 and E333 of yCGL and the corresponding F55 and Y338 of eCBL, proposed to be determinants of specificity. The PLP cofactor (yellow) is shown in covalent linkage to K203 of yCGL (grey) (Clausen et al., 1996; Messerschmidt et al., 2003). 
variants was decreased, their ability to complement the $\Delta m e t B$ and $\Delta m e t C$ strains was not improved, compared to the wild-type enzymes, demonstrating that these two residues alone are not sufficient as determinants of $\gamma$-elimination reaction specificity (Farsi et al., 2009).

In addition to a role as determinants of reaction specificity, the two glutamate residues in the active site of $\mathrm{YCGL}, \mathrm{E} 48$ and E333, were proposed to interact with the distal amino group of L-Cth (Messerschmidt et al., 2003). When E48 was substituted with either alanine or aspartate, the kinetic parameters of these variants were within 5-9-fold of the wild-type enzyme, indicating that this residue does not play a central role in substrate binding or catalysis (Farsi et al., 2009). Although the corresponding eCGS-D45 has also been proposed to have a role in substrate binding, via interaction with the $\alpha$ amino group of the enzyme's L-Cys substrate, replacement of this residue with alanine did not alter the $K_{\mathrm{m}}^{\text {LCys }}$, thereby demonstrating that this residue does not participate in LCys binding (Clausen et al., 1998; Jaworski et al., 2012). However, the 160-fold decrease in $k_{\text {cat }} / K_{\mathrm{m}}^{\text {L-OSHS }}$ of the D45F variant of eCGS is dominated by a decrease in $k_{\text {cat }}$ (Farsi $e t$ al., 2009), suggesting that this residue does contribute to maintenance of active-site architecture (Farsi et al., 2009). A role for residue yCGL-E333 in interaction with the distal amino group of L-Cth was supported by the inability to saturate variants of this residue within the solubility limit of this substrate (Farsi et al., 2009). In contrast, sitedirected variants of the corresponding eCGS-E325, proposed to interact with the $\alpha$-amino group of the L-Cys substrate (Clausen et al., 1998), had $K_{\mathrm{m}}{ }^{\mathrm{L}-\mathrm{Cys}}$ values within 3-fold of 
the wild-type, revealing that it is not involved in the binding of this substrate (Jaworski et al., 2012).

Residues R51 and R108 of yCGL are proposed to interact with the distal carboxylate group of L-Cth (Messerschmidt et al., 2003). The corresponding R48 and R106 of eCGS and eCBL-R58 have been confirmed to interact with the distal carboxylate groups of the L-OSHS and L-Cth substrates, respectively (Jaworski et al., 2012; Lodha et al., 2010). Residue R369 is predicted to bind the $\alpha$-carboxylate of the L-Cth substrate (Messerschmidt et al., 2003). A similar role has been confirmed for the corresponding positions R361 of eCGS and R372 of eCBL (Jaworski et al., 2012; Lodha et al., 2010). Clausen et al. (1998) proposed that residue R49 of eCGS may interact with the $\alpha$ carboxylate of its L-Cys substrate (Clausen et al., 1998). However, this hypothesis is not supported by the observation that substitution of this residue with alanine or lysine does not significantly alter the $K_{\mathrm{m}}^{\mathrm{L}-\mathrm{Cys}}$ of the enzyme from the wild-type (Jaworski et al., 2012). Similar results were reported for the corresponding R59 of eCBL as replacement of this residue with alanine or lysine had an effect of less than one order of magnitude on the kinetic parameters for L-Cth hydrolysis and inhibition by aminoethoxyvinylglycine (AVG), demonstrating that this residue does not participate in substrate binding or catalysis (Lodha et al., 2010). In yCGL, this position corresponds to a serine residue, S52. Given that the corresponding residues in eCGS and eCBL do not play roles in substrate binding, it is unlikely that the corresponding yCGL-S52 does. However, differences in identity of active-site residues such as this may hold clues to differences in substrate and reaction specificity among the enzymes of the transsulfuration pathways, hence the role of this residue in yCGL will be investigated. 
The crystal structure of yCGL indicates that residue Y49 forms a hydrogen bond to the phosphate of the PLP cofactor (Figure 1.5; Messerschmidt et al., 2003). This residue is conserved as a tyrosine in all fold type I enzymes (Tan et al., 1998), and the corresponding eCGS-Y46 and eCBL-Y56 residues have been proposed to bind the cofactor, to tether the catalytic base, and to impact substrate binding (Clausen et al., 1998; Clausen et al., 1997). Substitution of this residue with phenylalanine in both eCGS and $\mathrm{eCBL}$ yielded changes in catalytic efficiency of similar magnitude, dominated by a 20-fold increase in $K_{\mathrm{m}}$ (Jaworski et al., 2012; Lodha and Aitken, 2011). This increase in $K_{\mathrm{m}}$ upon removal of this residue's ability to hydrogen-bond suggests some involvement in substrate binding, but the observed changes may be due to loss of interaction with the phosphate moiety of PLP as the cofactor forms a covalent complex with the substrate (Jaworski et al., 2012).

Residue Y103 lies almost coplanar with the pyridine ring of PLP and is proposed to be essential for activating the incoming amino acid substrate for transaldimination (Messerschmidt et al., 2003). The $\pi$-stacking interaction between an aromatic side chain in this position and the pyridine ring of PLP are common to most fold type I PLPdependent enzymes (Hayashi, 1995). However, the specific nature of this residue (Trp, Tyr, Phe or His) may be a determinant of substrate and/or reaction specificity and its ability to participate in binding or catalysis may be determined by the identity and mobility of other active-site residues (Hayashi, 1995; Lodha and Aitken, 2011). The position of this residue compared to that of eCBL-Y111 is slightly different (Figure 1.8), but Messerschmidt et al. (2003) hypothesized that differences in the flexibility of this region would not affect the proposed role of this residue in catalysis. 


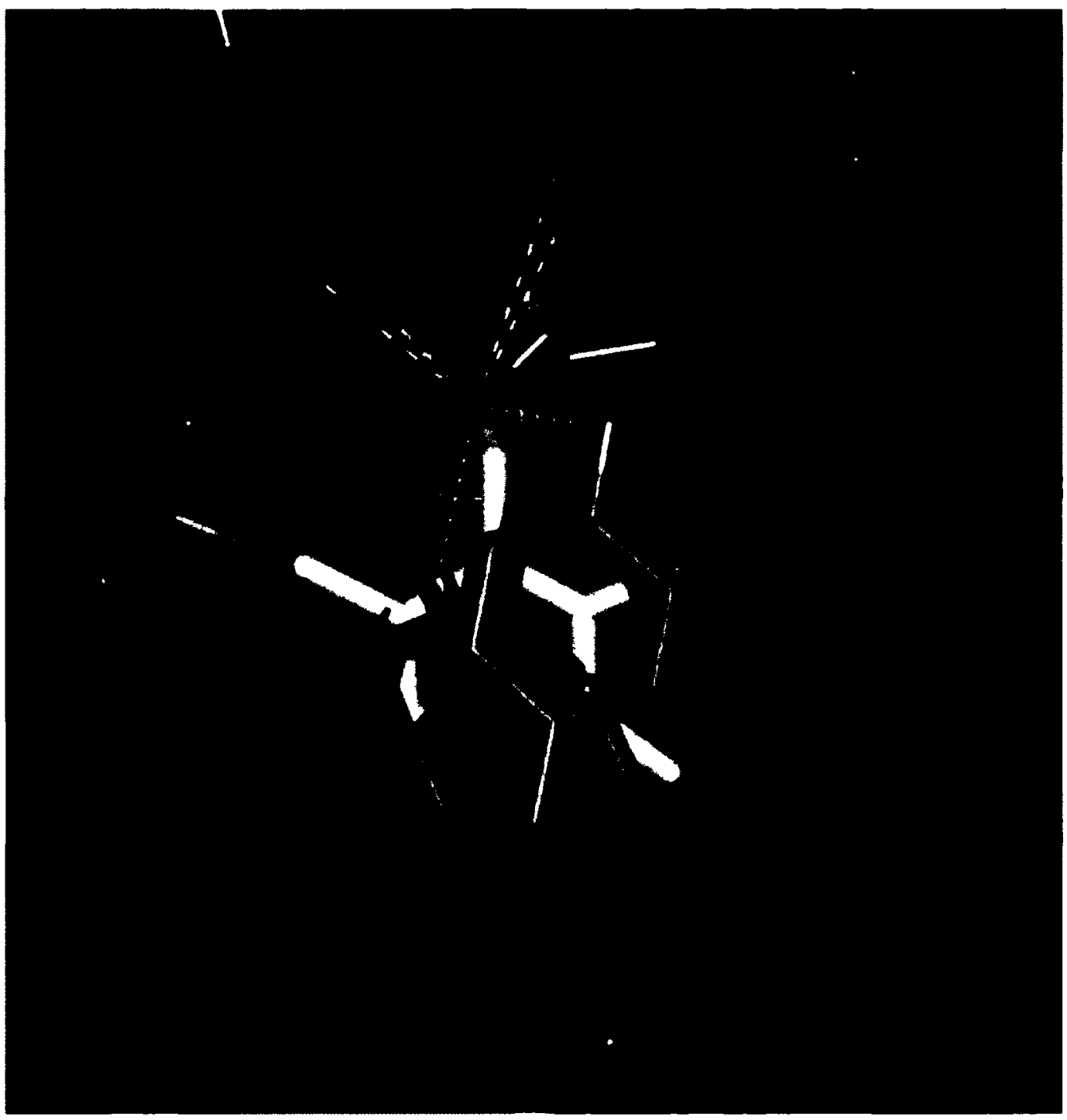

Figure 1.8. Overlay of active-sites of yCGL (magenta; PDB IN8P) and eCBL (blue; PCB 1CL1) showing conserved residues with putative roles in catalysis. The PLP cofactor (yellow) is shown in covalent linkage to K203 of yCGL (grey), and dotted lines indicate distances between heteroatoms in angstroms. (Clausen et al., 1996; Messerschmidt et al., 2003) 
The crystallographic data suggest that the nearby guanidinium group of R51 and the protonated aldimine could lower the $\mathrm{p} K_{\mathrm{a}}$ of $\mathrm{Y} 103$, allowing it to participate in catalysis by abstracting a proton from the incoming substrate (Messerschmidt et al., 2003). A similar role was proposed for eCBL-Y $111 \mathrm{~F}$ due the proximity of its hydroxyl group to $C_{\alpha}$ of the substrate (Clausen et al., 1996). However, substitution of the corresponding residue Y111 of eCBL to phenylalanine resulted in only a 2.3-fold reduction and 3.6-fold increase in $k_{\text {cat }}$ and $K_{\mathrm{m}}{ }^{\mathrm{L}-\mathrm{Cth}}$, respectively, and the $k_{\text {cat }} / K_{\mathrm{m}}{ }^{\mathrm{L}-\mathrm{Cth}}$ versus $\mathrm{pH}$ profile was unchanged from that of the wild-type enzyme, demonstrating that this residue does not participate in proton transfer in the $\beta$-elimination reaction catalyzed by eCBL (Lodha and Aitken, 2011). However, this may not be the case in yCGL, because the $\gamma$-elimination reaction requires proton transfers to $C_{\beta}$ and $C 4$ ' that are not part of the $\beta$-elimination mechanism of CBL. The $\mathrm{p} K_{\mathrm{a}}$ values of the basic limbs of the eCBL and yCGL specific activity versus $\mathrm{pH}$ profiles are 10.3 and 7.7 , respectively (Farsi et al., 2009). While the $\mathrm{p} K_{\mathrm{a}}$ of 10.3 may correspond to eCBL-Y111, the lower $\mathrm{p} K_{\mathrm{a}}$ value of yCGL suggests that either the $\mathrm{p} K_{\mathrm{a}}$ of the corresponding $\mathrm{Y} 103$ residue is lowered, or deprotonation of a residue is required in yCGL that is not necessary for the eCBL reaction.

A substrate-binding role for eCBL-Y111 in interacting with the distal amino moiety of L-Cth was also proposed, based on the structure of eCBL in complex with the inhibitor aminoethoxyvinylglycine (AVG) (Clausen et al., 1997). However, the minor 26-fold changes observed in $K_{m}{ }^{\mathrm{L}-\mathrm{Cth}}$ for the hydrolysis of L-Cth, as well as the alternate substrates L-Cys and L-OAS, which lack a distal amino group, indicate that in solution this residue may be positioned in a way that does not cause it to interact with the 
substrate, contrary to what is suggested by the crystal structure (Lodha and Aitken, 2011). A similar role in binding the L-OSHS substrate was proposed for the corresponding eCGS-Y101 (Clausen et al., 1998), but was not supported by the modest, 2-fold change in $K_{\mathrm{m}}^{\mathrm{L}}{ }^{\mathrm{LOSHS}}$ of the Y101F variant (Jaworski et al., 2012).

Another tyrosine residue in $\mathrm{CCBL}, \mathrm{Y} 238$, was also proposed to interact with the $\mathrm{L}$ Cth substrate (Clausen et al., 1997). When this residue was replaced with phenylalanine, a 16-fold increase in $K_{\mathrm{m}}{ }^{\mathrm{L}-\mathrm{Cth}}$ was observed, while the $K_{\mathrm{i}}^{\mathrm{AVG}}$ for inhibition with AVG and $K_{\mathrm{m}}$ values for hydrolysis of L-Cys and L-OAS, both of which lack a second carboxylate group, increased only 2-2.5-fold, thus supporting a role for this residue in binding the distal carboxylate of L-Cth (Lodha and Aitken, 2011). However, while CBL and CGL both bind the same pseudosymmetric substrate, in yCGL this tyrosine (eCBL-Y238) residue is replaced by an asparagine (yCGL-N232). Therefore, this residue will be interesting to examine as a potential determinant of reaction specificity in yCGL.

In CGS, CBL, and CGL, a conserved serine residue in the active site tethers the catalytic lysine, following the transaldimination reaction that releases the $\varepsilon$-amino group of this residue from the Schiff-base linkage with the cofactor (Figure 1.8; Clausen et al., 1998; Clausen et al., 1996; Messerschmidt et al., 2003). In eCBL, S339 tethers the catalytic $\mathrm{K} 210$, as indicated by the 5600 -fold reduction in $k_{\text {cat }}$ of the S339A variant, with a $K_{\mathrm{m}}{ }^{\mathrm{L}-\mathrm{Cth}}$ increase of only 2-fold (Lodha and Aitken, 2011). This serine residue, through the interaction between its hydroxyl group and the $\varepsilon$-amino group of $\mathrm{K} 210$, acts as a determinant of reaction specificity by restricting the movement of the catalytic base to prevent protonation at C4' of the cofactor (Lodha and Aitken, 2011). When the corresponding eCGS residue S326 was replaced with alanine, a 40-fold and 360-fold 
decrease in $k_{\text {cat }}$ was observed for the $\gamma$-elimination and $\gamma$-replacement, respectively, of the L-OSHS substrate (Jaworski et al., 2012). The 5600 -fold reduction in $k_{\text {cat }}$ for the CBLcatalyzed $\beta$-elimination of L-Cth, versus the 360 -fold reduction in $k_{\text {cat }}$ for the $\gamma$ elimination of L-OSHS catalyzed by the corresponding eCGS-S326A variant, indicates that the catalytic lysine must be more tightly restrained in $\mathrm{CBL}$ to prevent protonation of C4' of PLP, as expected given that this step is required by the $\gamma$-elimination/replacement mechanism of CGS (Lodha and Aitken, 2011). Given that yCGL and eCGS both possess $\gamma$-elimination activity, a reduction in $k_{\text {cat }}$ similar to those observed for the eCGS-S326A mutant would be anticipated for substitution of the corresponding S334 residue of yCGL with alanine.

\subsection{Reaction Mechanism.}

The reaction mechanism of yCGL-catalyzed $\gamma$-elimination of L-Cth (Figure 1.9) is identical to that of the CGS-catalyzed $\gamma$-elimination of L-OSHS, which is an undesirable side reaction of this enzyme observed in the absence of the L-Cys substrate (Brzovic et al., 1990; Aitken et al., 2003). For L-Cth to bind productively to the PLP-enzyme complex, a general base, proposed to be $\mathrm{Y} 103$, abstracts a proton from the $\alpha$-amino group of the incoming substrate to initiate transaldimination (reaction 1) with the internal aldimine (intermediate I) to result in formation of the external aldimine (intermediate II). Following transaldimination, $\mathrm{K} 203$, the cataltytic base, abstracts the proton from $\mathrm{C}_{\boldsymbol{\alpha}}$ of the substrate (reaction 2) to generate a short-lived quinonoid intermediate (III), and then facilitates proton transfer to C4' of the cofactor (reaction 3) to produce the PLP-L-Cth 

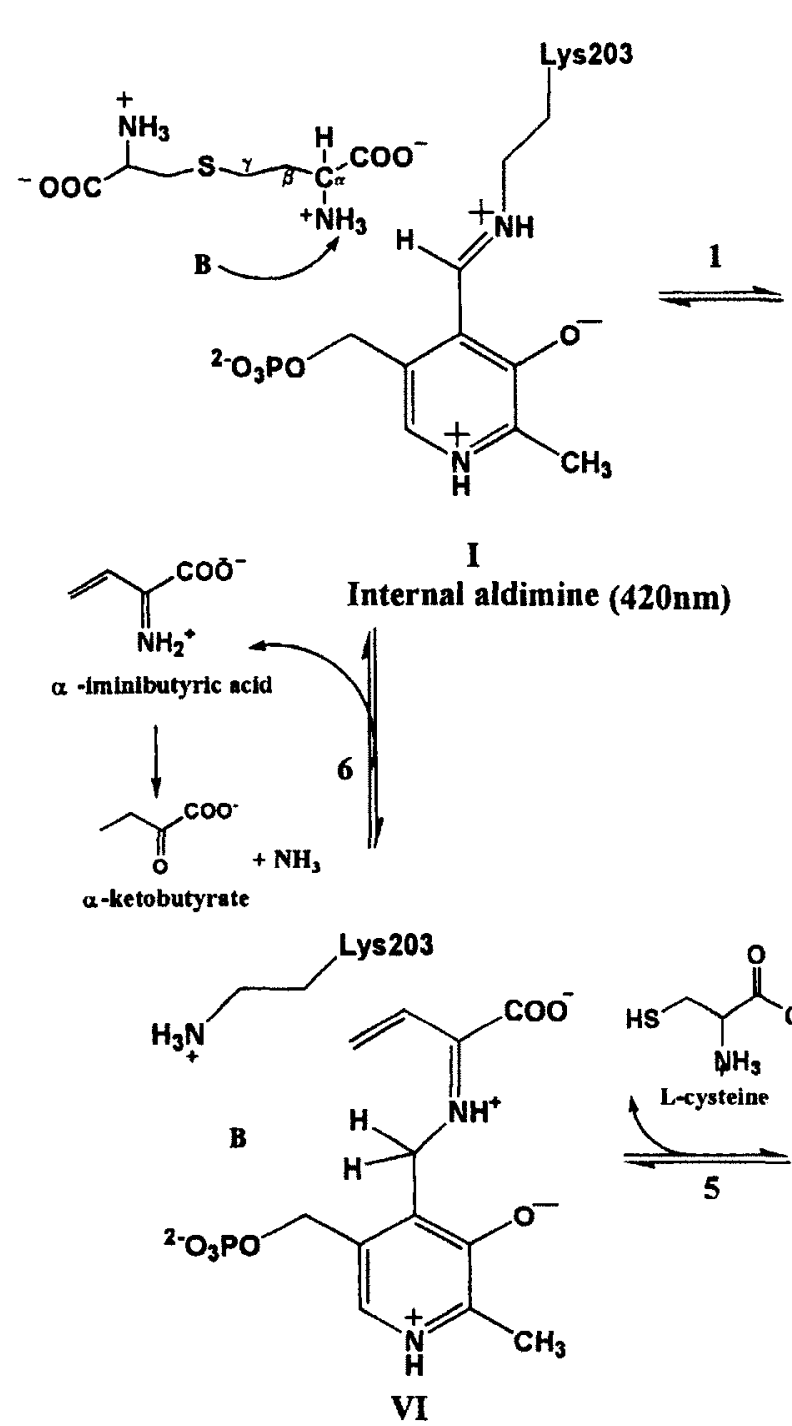

$\beta \gamma$-unsaturated ketimine

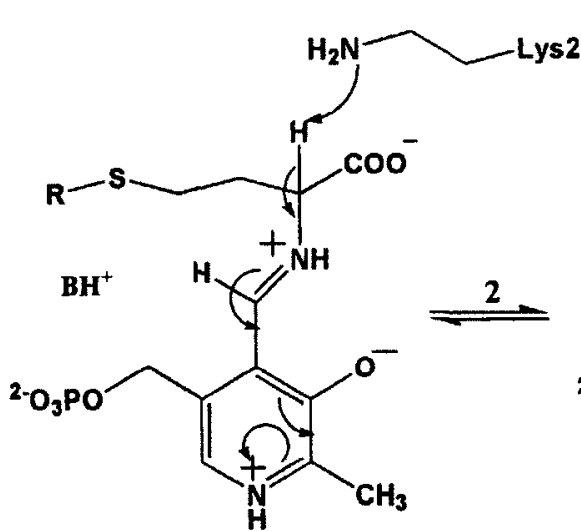

II

External aldimine of $\mathrm{L}-\mathrm{Cth}$

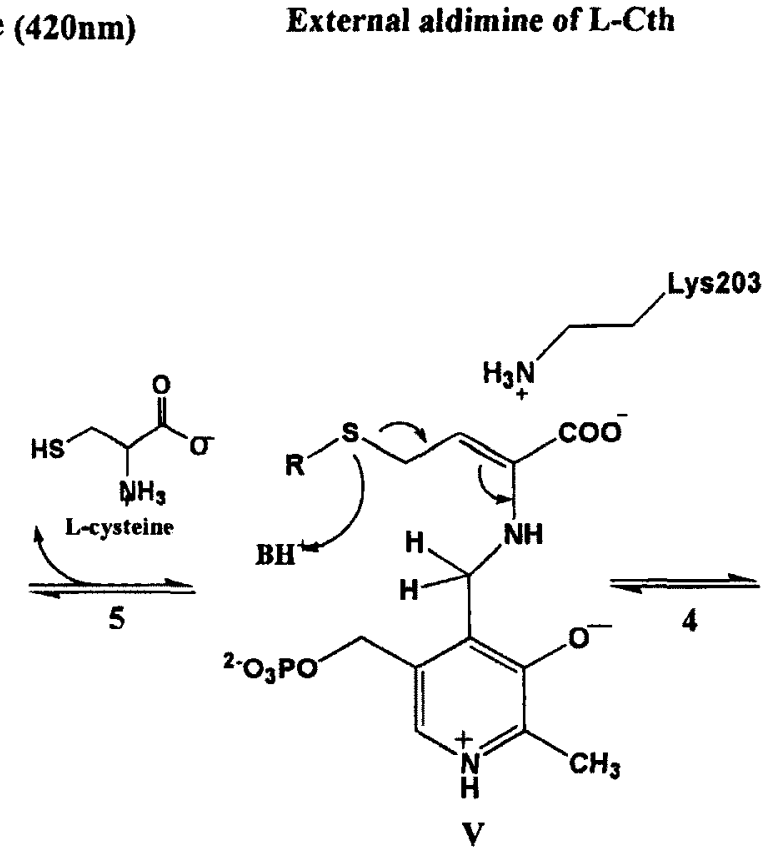

$\alpha \beta$-unsaturated ketimine

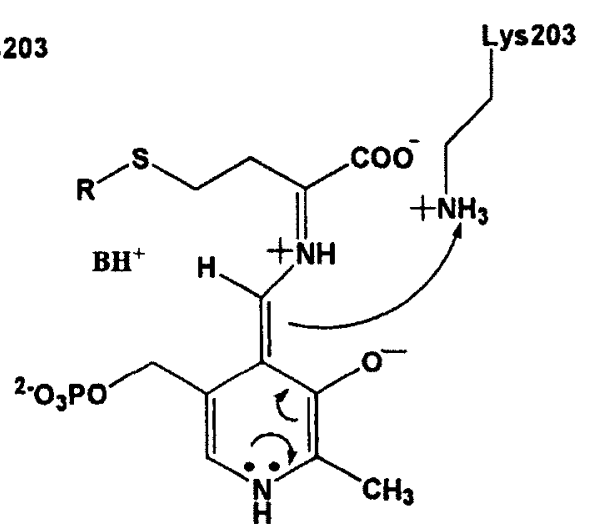

III

Quinonoid internediate

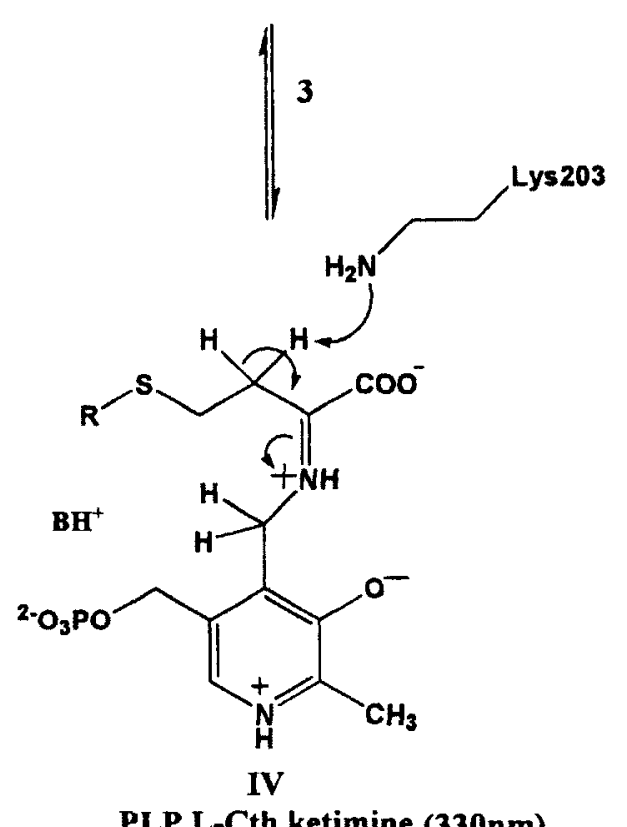

PLP L-Cth ketimine (330nm)

Figure 1.9. Proposed reaction mechanism of yCGL-catalyzed $\gamma$-elimination of L-Cth. Characteristic wavelengths of maximum absorbance are indicated for key intermediates (Brzovic et al., 1990; Aitken et al., 2003; Messerschmidt et al., 2003). 
ketimine intermediate (IV). The conformationally flexible K203 subsequently abstracts a proton from $C_{\beta}$ of the substrate to initiate $\gamma$-cleavage (reaction 4 ), and then a general base, potentially Y103, facilitates the release of the L-Cys product (reaction 5). The unsaturated ketimine intermediate (VI) subsequently undergoes transaldimination with the $\varepsilon$-amino of K203 to re-form the internal aldimine (intermediate I) and release $\alpha$-iminibutyric acid (reaction 6), which is hydrolyzed in an aqueous environment to produce $\alpha$-ketobutyrate and ammonia.

\subsection{Role of CGL in Cystathioninuria.}

Cystathioninuria (MIM 219500), an autosomal recessive condition in which L-Cth accumulates to abnormally high levels in blood plasma, is linked to mutations in the gene encoding hCGL (Wang and Hegele, 2003). Cystathioninuria is associated with a wide range of conditions, such as developmental delay, neuroblastoma, and Down's syndrome, and affects $\sim 1: 14,000$ live births (Wang and Hegele, 2003). The specific mutations of the gene encoding CGL that cause cystathioninuria are varied, and this is reflected in a range of patient responses to vitamin $B_{6}$ treatment (Wang and Hegele, 2003). Mutations causing cystathioninuria have been identified that affect both PLP-binding and oligomerization of the CGL tetramer (Kraus et al., 2009; Zhu et al., 2008). 


\subsection{Role of CGL in Hydrogen Sulfide Production.}

Both CBS and CGL have attracted interest in recent years due to their ability to produce hydrogen sulfide $\left(\mathrm{H}_{2} \mathrm{~S}\right)$ gas (Abe and Kimura, 1996; Dominy and Stipanuk, 2004; Yang et al., 2008). This gas, usually toxic, has been found to be present at relatively high endogenous levels of $1.5 \mu \mathrm{g} / \mathrm{g}$ in the brain (Warenycia et al., 1989), suggesting a physiological function. Other gaseous molecules including $\mathrm{NO}$ and $\mathrm{CO}$ have been shown to have roles in learning and memory processes via their involvement in long-term potentiation, leading to speculation that $\mathrm{H}_{2} \mathrm{~S}$ may also be involved in neuromodulation (Abe and Kimura, 1996). Both CBS mRNA and protein are expressed highly in the cerebellum and hippocampus, while CGL expression is not detectable in brain by northern blot analysis (Abe and Kimura, 1996). Levels of $\mathrm{H}_{2} \mathrm{~S}$ in the brains of CGL knockout mice are the same as the wild-type, confirming that CGL is not the source of $\mathrm{H}_{2} \mathrm{~S}$ in the brain (Yang et al., 2008). Production of $\mathrm{H}_{2} \mathrm{~S}$ in the brain is inhibited by hydroxylamine (a CBS inhibitor), but not propargylglycine (a CGL inhibitor), and increased by SAM (a CBS activator) (Abe and Kimura, 1996). These results suggest that CBS is the major enzyme contributing to endogenous $\mathrm{H}_{2} \mathrm{~S}$ production in brain (Abe and Kimura, 1996). The production of $\mathrm{H}_{2} \mathrm{~S}$ by $\mathrm{CBS}$ occurs via a $\beta$-replacement reaction using L-Hcys and L-Cys as substrates (Chen et al., 2004). Hydrogen sulfide has been shown to enhance $N$-methyl-D-aspartate receptor-mediated responses in brain, facilitating nerve potentiation associated with memory (Abe and Kimura, 1996). In brains of patients with Alzheimer's disease, both $\mathrm{H}_{2} \mathrm{~S}$ and SAM levels are low, and homocysteine levels are increased, consistent with loss of CBS function (Eto et al., 2002). Thus a decrease in $\mathrm{H}_{2} \mathrm{~S}$ 
from loss of CBS activity in the brain may be associated with some symptoms of Alzheimer's disease such as cognitive decline (Eto et al., 2002).

In the peripheral vascular system, $\mathrm{H}_{2} \mathrm{~S}$ is proposed to have a role in regulating hypertension (Siebert et al., 2008; Yang et al., 2008; Yang et al., 2010). In vivo studies using CGL-knockout mice established that CGL localizes to blood vessel endothelium, and that CGL-deficient mice had increased plasma levels of homocysteine and decreased cysteine levels, as well as reduced serum levels of $\mathrm{H}_{2} \mathrm{~S}$ and lower $\mathrm{H}_{2} \mathrm{~S}$ production rates in the heart and aorta (Yang et al., 2008). These knockout mice showed reduced endothelium-dependent vasorelaxation, and developed hypertension (Yang et al., 2008). These results suggested that CGL is the physiological source of $\mathrm{H}_{2} \mathrm{~S}$ in vascular tissues; however Siebert and colleagues (2008) found both CBS and CGL mRNA and protein expressed in the aorta, vena cava, hepatic artery and portal vein, while in the intrahepatic terminal branches of the hepatic artery and portal vein, only CGL was expressed, indicating that $\mathrm{H}_{2} \mathrm{~S}$-producing enzymes may be differentially expressed in the vascular system (Siebert et al., 2008). So while most studies of enzyme-produced $\mathrm{H}_{2} \mathrm{~S}$ in the vascular system have focused on CGL, additional information may be gleaned by investigating the roles of both enzymes of the reverse transsulfuration pathway.

The ability of $\mathrm{H}_{2} \mathrm{~S}$ to relax smooth muscles in the vascular system was proposed to be mediated by the opening of ATP-sensitive potassium channels (Yang et al., 2008). This was confirmed by the observation that application of glibenclamide, a selective inhibitor of $\mathrm{K}^{+} \mathrm{ATP}$ channels, inhibited vasorelaxation of the hepatic artery by $\mathrm{H}_{2} \mathrm{~S}$ (Siebert et al., 2008). Hydrogen sulfide also affects cellular proliferation and apoptosis, by arresting the cell in G1 phase via multiple cell cycle regulators (Yang et al., 2010). 
The increased proliferation both in vitro and in vivo of vascular smooth muscle cells of CGL-deficient mice could lead to thickening of blood vessel walls, causing the hypertension that is observed in CGL knockout mice (Yang et al., 2010).

The low substrate specificity of CGL allows it to catalyze, in addition to its primary $\alpha, \gamma$-elimination of L-Cth, the production of $\mathrm{H}_{2} \mathrm{~S}$ by a variety of potential mechanisms from L-Hcys and/or L-Cys substrates (Figure 1.10; Chiku et al., 2009; Huang et al., 2010). Although the primary source of endogenous $\mathrm{H}_{2} \mathrm{~S}$ has been proposed to be the desulfhydrylation via $\beta$-elimination of L-Cys, kinetic studies of human CGL have revealed that at saturating concentrations, L-Hcys is a better substrate for $\mathrm{H}_{2} \mathrm{~S}$ production by CGL than L-Cys (Chiku et al., 2009). The human CGL enzyme is capable of producing $\mathrm{H}_{2} \mathrm{~S}$ by $\gamma$-replacement of L-Hcys to give homolanthionine, by $\gamma$-elimination of L-Hcys to give $\alpha$-ketobutyrate, and by $\beta$-elimination of L-Cys, with catalytic efficiencies of 830,350 and $270 \mathrm{M}^{-1} \mathrm{~s}^{-1}$, respectively, and the former is proposed to be the predominant source of endogenous $\mathrm{H}_{2} \mathrm{~S}$ (Chiku et al., 2009). The $K_{\mathrm{m}}{ }^{\mathrm{L}-\mathrm{Cth}}$ is $\sim 10$-fold lower than the $K_{\mathrm{m}}^{\text {L-Hcys }}$ for hCGL, and the catalytic efficiency of the physiological $\gamma$ elimination of L-Cth is 20 and 30-fold higher than the elimination reactions to give $\mathrm{H}_{2} \mathrm{~S}$ from L-Hcys and L-Cys, respectively (Chiku et al., 2009). In simulating substrate availability of the cellular environment, at normal $(10 \mu \mathrm{M})$ homocysteine levels, the $\beta$ elimination of cysteine appears to be main contributor $(70 \%)$ to $\mathrm{H}_{2} \mathrm{~S}$ production, followed by the $\gamma$-elimination of homocysteine $(29 \%)$. However, at concentrations of homocysteine $(200 \mu \mathrm{M})$ such as those seen in severe hyperhomocysteinemia, the $\gamma$ elimination of homocysteine becomes the predominant source of $\mathrm{H}_{2} \mathrm{~S}$ (Chiku et al., 2009 ), leading to the proposal that in vivo substrate availability is the main factor 

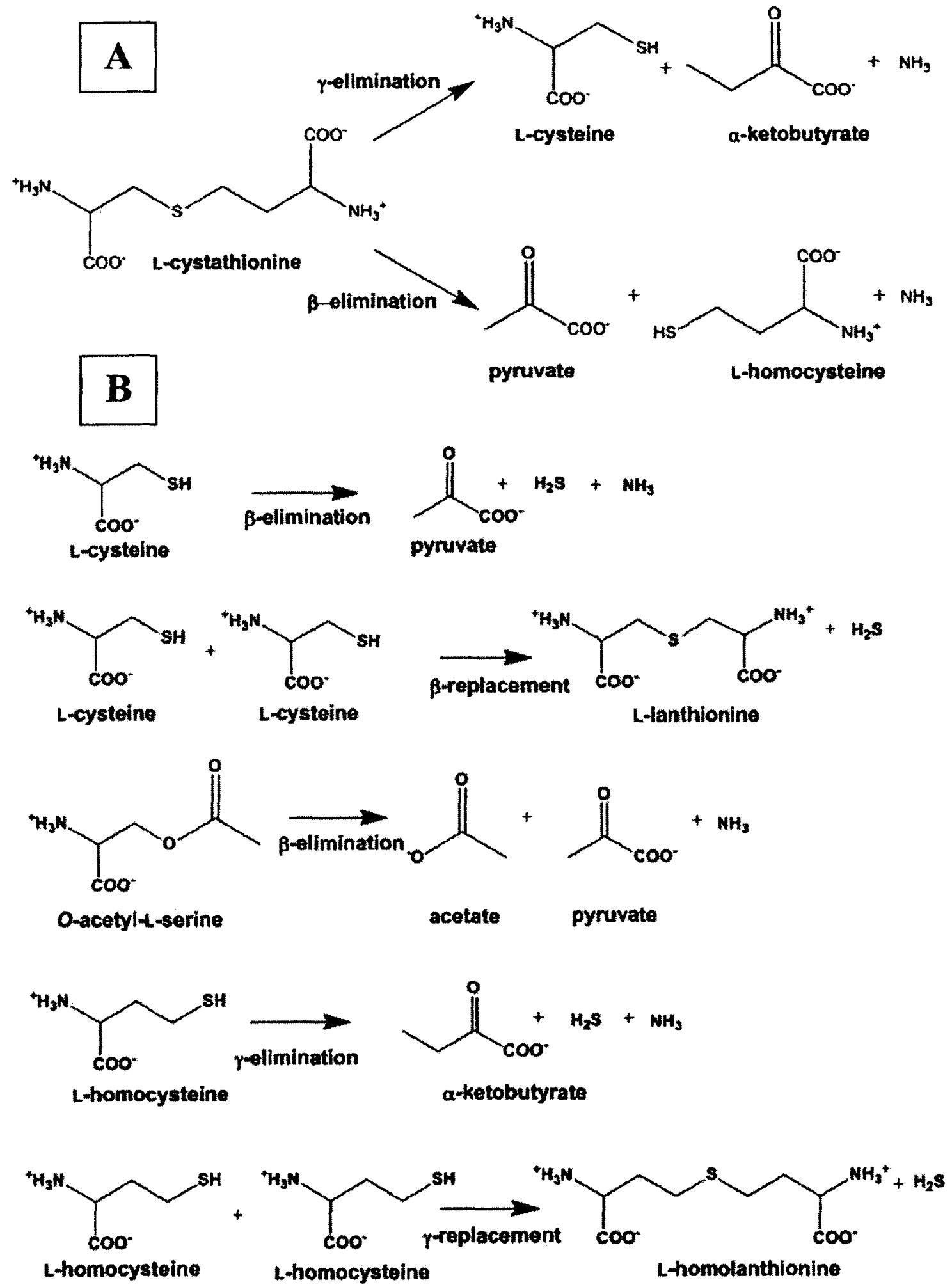

Figure 1.10. The various reactions, and their respective products, catalyzed by yCGL on (A) the physiological L-Cth substrate and (B) the alternative substrates L-Cth, L-OAS, and L-Hcys. Adapted from Chiku et al. (2009). 
regulating the partitioning of CGL activity to different reaction pathways. However, the assays employed in the 2009 study by Chiku and colleagues were not sufficient to differentiate between $\beta$ - versus $\gamma$-elimination or replacement reactions, indicating that caution must be taken when interpreting the kinetic parameters obtained, as well as highlighting the need for additional characterization of the substrate and reaction specificity of CGL.

Based on the crystal structure of yCGL, residue E333 was proposed to be a central determinant of reaction specificity (Messerschmidt et al., 2003). A role for the corresponding E325 residue of eCGS in modulating reaction specificity is supported by the increase in absorbance at $330 \mathrm{~nm}$, characteristic of pyridoxamine (PMP) formation, in the absorbance spectra of the eCGS-E325A/Q variants after incubation with L-alanine, which can undergo transamination or racemization but not elimination or substitution (Figure 1.11; Jaworski et al., 2012). Huang et al. (2010) determined that the site-directed substitution of hCGL-E339, corresponding to yCGL-E333, with alanine results in a 6fold increase in $\mathrm{H}_{2} \mathrm{~S}$ production compared to the wild-type enzyme. This residue was also replaced with other hydrophobic residues, and an increase in the $\alpha, \beta$-elimination of cysteine, to produce $\mathrm{H}_{2} \mathrm{~S}$, was observed, suggesting that the hydrophobicity of this residue, as opposed to its specific identity, determines the rate of $\mathrm{H}_{2} \mathrm{~S}$ production (Huang et al., 2010).

Residue Y103 is proposed to deprotonate the $\alpha$-carbon of the incoming substrate, allowing it to bind the PLP cofactor (Messerschmidt et al., 2003). When this residue in the human enzyme (hCGL-Y114) was replaced with phenylalanine, a 3.6-fold increase in $\mathrm{H}_{2} \mathrm{~S}$ production was observed compared to the wild-type enzyme, indicating that the 


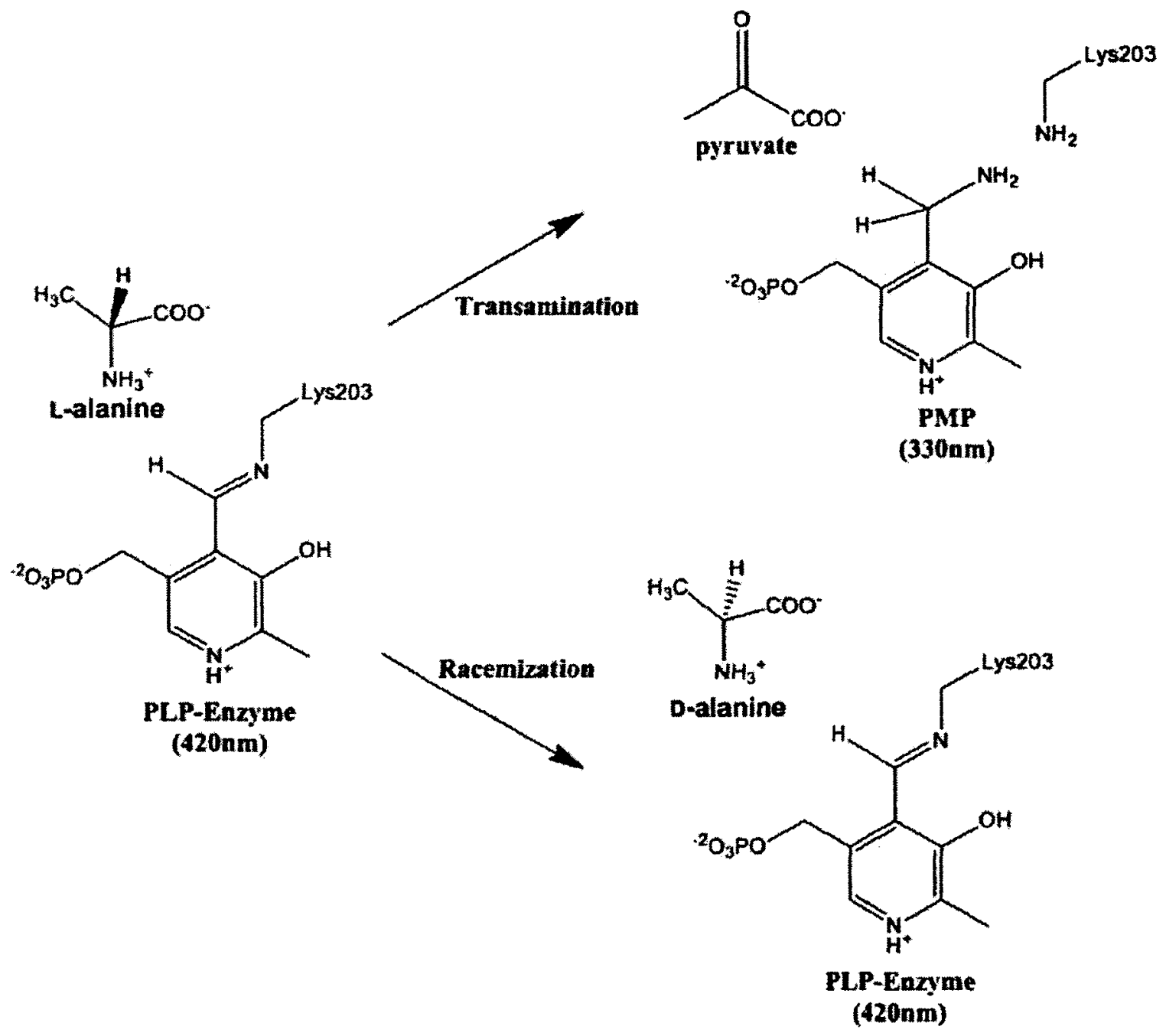

Figure 1.11. The PLP-dependent transformations possible for L-alanine. The products arising from (A) the first half-reaction of a transamination, or (B) racemization are depicted in addition to the wavelength of maximum absorbance of characteristic intermediates (Kirsch et al., 1984; Toney, 2011). The residue numbering used is that of yCGL. 
hydroxyl group of the tyrosine side chain is not required for $\mathrm{H}_{2} \mathrm{~S}$ production (Huang et al., 2010). However, the $\pi$-stacking interactions provided by the aromatic ring are necessary for productive active-site geometry, as indicated by the inability of the hCGLY114A variant to produce $\mathrm{H}_{2} \mathrm{~S}$ (Huang et al., 2010). This tyrosine may not be involved in directly binding the substrate, but removal of the hydroxyl group of this residue appears to alter active-site dynamics in the context of both the eCBL and hCGL active sites. Minor changes in kinetic parameters are observed for the phenylalanine substitution variant of the corresponding Y111 residue in $\mathrm{eCBL}$, indicating that the hydroxyl group is also not required for L-Cth hydrolysis in this enzyme (Lodha and Aitken, 2011). However, while the $k_{\text {cat }}$ and $K_{\mathrm{m}}^{\mathrm{L}-\mathrm{Cys}}$ for the hydrolysis of L-Cys by the eCBL-Y111F variant are similar to the wild-type, the $K_{\mathrm{i}}^{\mathrm{L}-\mathrm{Cys}}$ is decreased 7.5 -fold, indicating that the active-site architecture may be altered to allow a second L-Cys molecule to bind (Lodha and Aitken, 2011). Therefore, the role of residue Y103 of yCGL will be investigated as a determinant of substrate and reaction specificity. 


\subsection{Objective and Hypotheses.}

The structures of eCGS, eCBL and yCGL are so similar that $\sim 350 \mathrm{C}_{\alpha}$ atoms of their backbones can be superimposed with an r.m.s. deviation of only $\sim 1.5 \AA$ (Messerschmidt et al., 2003), yet these enzymes exhibit distinct substrate and reaction specificities. Based on available crystal structures and the demonstrated roles of corresponding residues of $\mathrm{eCGS}$ and $\mathrm{eCBL}$, it is possible to make predictions about the functions of various active-site residues in CGL (Clausen et al., 1996; Clausen et al., 1997; Clausen et al., 1998; Messerschmidt et al., 2003; Aitken et al., 2003; Farsi et al., 2009; Lodha et al., 2010; Lodha and Aitken, 2011; Jaworski et al., 2012). However, these roles have yet to be confirmed. The goal of this research is to elucidate, via site-directed substitution, the roles of active-site residues in the model yeast CGL enzyme. The hypotheses guiding this study are:

1. residues R51, S52, R108, N232 and R369 interact with the L-Cth substrate;

2. residue Y49 participates in substrate binding, and Y103 and S334 facilitate catalysis and are determinants of specificity in yCGL;

3. residues E48 and E333 interact with L-Cth and are determinants of substrate specificity in yCGL.

The knowledge gained from this investigation will contribute to the design of antibiotic and herbicidal agents that are specific to bacterial and plant transsulfuration enzymes, as well as further a more detailed understanding of the involvement of CGL in hydrogen sulfide biogenesis and cystathioninuria that will aid medical applications such as treatment of hypertension and metabolic disorders. 


\section{METHODS}

\subsection{Reagents.}

Hydroxyisocaproate dehydrogenase (HO-HxoDH) was purified as described previously (Morneau et al., 2012). L-Lactate dehydrogenase (LDH), $\beta$-nicotinamide adenine dinucleotide ( $\beta$-NADH, reduced form), O-acetyl-L-serine (L-OAS), L-Cys, Lhomocysteine thiolactone and L-Cth [S-(2-amino-2-carboxyethyl)-L-homocysteine] were Sigma products, and L-Hcys was prepared from the thiolactone as in Kashiwamata and Greenberg (1970). AMPSO (3-[1,1-dimethyl-2-hydroxyethyl)amino]-2hydroxypropanesulfonic acid) and TAPS (N-Tris(hydroxymethyl)methyl-3aminopropanesulfonic acid) were from Sigma, and CAPS ( $N$-cyclohexyl-3aminopropanesulfonic acid), and MOPS (3-(N-morpholino)propanesulfonic acid) were

obtained from Fisher Scientific. 5,5'-Dithiobis(2-nitrobenzoic acid) (DTNB) was obtained from Pierce and nickel-nitriloacetic acid (Ni-NTA) resin was from Qiagen. Oligonucleotide primers were synthesized by Integrated DNA Technologies and sitedirected variants were sequenced by BioBasic prior to expression.

\subsection{Construction, expression and purification of site-directed mutants.}

Oligonucleotide primers (Table 2.1) were designed to introduce targeted point mutations in the nucleotide sequence of yCGL (Genbank accession no. D14135). Overlap-extension polymerase chain reaction (OE-PCR) amplification (Higuchi et al., 1988) of the yCGL wild-type template was carried out using the primers in Table 2.1. The E48A/E333A, E48D/E333D and E48Q/E333Q mutants were also constructed by 
Table 2.1. Primers used in the construction of site-directed mutants of yCGL.

\begin{tabular}{|c|c|}
\hline Primer & Sequence \\
\hline $\mathrm{E} 48 \mathrm{Q}^{\mathrm{a}}$ & 5'-ctatcggtacttaccagtactccagatc \\
\hline $\mathrm{Y}_{49 \mathrm{~F}^{\mathrm{a}}}$ & 5'-cggtacttacgaattttccagatctc \\
\hline $\mathrm{R}^{2} \mathrm{~A}^{\mathrm{a}}$ & 5'-cttacgaatactccgcgtctcaaaatcc \\
\hline $\mathrm{R}^{2} 51 \mathrm{~K}^{\mathrm{a}}$ & 5'-ggtacttacgaatactccaaatctcaaaatcc \\
\hline $\mathrm{S} 52 \mathrm{~A}^{\mathrm{a}}$ & 5'-cgaatactccagagcgcaaaatcctaac \\
\hline $\mathrm{S} 52 \mathrm{R}^{\mathrm{a}}$ & 5'-cgaatactccagacgccaaaatcctaac \\
\hline $\mathrm{Y} 103 \mathrm{~F}^{\mathrm{a}}$ & 5'- ggtgatgtgtttggtggtacc \\
\hline $\mathrm{R} 108 \mathrm{~A}^{\mathrm{a}}$ & 5'-gtggtacccacgcgtacttcac \\
\hline $\mathrm{R} 108 \mathrm{~K}^{\mathrm{a}}$ & 5'-cggtggtacccacaaatacttcacc \\
\hline $\mathrm{N} 232 \mathrm{~A}^{\mathrm{a}}$ & 5'-gttcttacaagcggccattgg \\
\hline $\mathrm{E}_{333 \mathrm{~A}^{\mathrm{a}}}$ & 5'-ctgttcacattggccgcgtcccttggtgg \\
\hline $\mathrm{E} 333 \mathrm{D}^{\mathrm{a}}$ & 5'-cattggccgattccettggtg \\
\hline $\mathrm{E} 333 \mathrm{Q}^{\mathrm{a}}$ & 5'-cattggcccagtcccttggtg \\
\hline $\mathrm{S} 334 \mathrm{~A}^{\mathrm{a}}$ & 5'- cattggccgaagcgcttg \\
\hline $\mathrm{R}_{369 \mathrm{~A}^{\mathrm{a}}}$ & 5'-gacgacttggttgcgatctctgtc \\
\hline $\mathrm{R} 369 \mathrm{~K}^{\mathrm{a}}$ & 5'-gacgacttggttaaaatctctgtcggtattgaag \\
\hline pSECseq0 & 5'-ggcgtcaggcagccatcggaagctg \\
\hline pSECseg7r & 5'- gcccgccaccctccgggccgttgcttcgc \\
\hline
\end{tabular}

${ }^{a}$ Second mutagenic primers are the reverse complement of the sequence listed. 
OE-PCR with the E333A, E333D and E333Q mutagenic primers, respectively (Table 2.1), but using the E48A, E48D and E48Q single mutants, respectively, as templates. Reassembly PCR was carried out using the pSECseq0 and pSECseq7r primers, which are specific for the $5^{\prime}$ and $3^{\prime}$ regions of the vector, respectively, flanking the yCGL coding region. Following purification, on a $1 \%$ agarose gel, the reassembled amplification products were digested with $\mathrm{NdeI}$ and SacI (New England Biolabs) and inserted at the corresponding position of the pTrc99aAF expression vector using T4 DNA ligase (New England Biolabs).

The pTrc99aAF vector encodes an N-terminal 6-histidine affinity tag and linker, containing a Factor Xa protease cleavage site, which does not alter the kinetic parameters of yCGL (Farsi et al., 2009). The expression constructs were transformed via the heatshock method into Escherichia coli ER1821 $\Delta$ metC cells, lacking the wild-type eCBL coding region to avoid the possibility of contamination with the $\beta$-lyase of the $E$. coli host, and nucleic acid sequencing confirmed the presence of the desired mutation in all site-directed yCGL constructs.

Expression and purification of the variant enzymes was performed as described by Farsi et al. (2009). Five $1 \mathrm{~L}$ batches of Luria Broth media in $2.8 \mathrm{~L}$ baffled Fernbach flasks were inoculated with a $100 \mathrm{~mL}$ overnight culture of cells expressing the yCGL construct at a 1:50 inoculum-to-media ratio and grown at $37^{\circ} \mathrm{C}$ until the $\mathrm{OD}_{600}$ reached 0.6 , at which point IPTG was added to a final concentration of $0.2 \mathrm{mM}$ to induce expression of the yCGL construct. The cells were grown for an additional $16 \mathrm{~h}$ at $37^{\circ} \mathrm{C}$, and harvested at $5,000 \mathrm{rpm}$ for $10 \mathrm{~min}$ at $4^{\circ} \mathrm{C}$. The cell pellets were washed in $100 \mathrm{~mL}$ of $0.85 \% \mathrm{NaCl}$, and centrifuged at $5,000 \mathrm{rpm}$ for an additional $10 \mathrm{~min}$ at $4^{\circ} \mathrm{C}$. The harvested 
cell pellets were resuspended in $130 \mathrm{~mL}$ lysis buffer $(50 \mathrm{mM}$ potassium phosphate, $\mathrm{pH}$ $7.8,10 \mathrm{mM}$ imidazole, $20 \mu \mathrm{M}$ PLP) with $1 \mathrm{mg} / \mathrm{mL}$ lysozyme and incubated at room temperature for $20 \mathrm{~min}$, followed by 6 cycles of sonication (VibraCell sonicator, Sonic and Material, Inc.) for $30 \mathrm{sec}$ at $50 \%$ duty cycle. After centrifugation at 17,000 rpm for $45 \mathrm{~min}$, the supernatant was loaded onto $3 \mathrm{~mL}$ of Ni-NTA resin (equilibrated with lysis buffer) in a $2.5 \times 10 \mathrm{~cm}$ column. The column was then washed with at least 20 column volumes of lysis buffer. The protein was eluted with a $200 \mathrm{~mL}$ linear gradient of 10-100 $\mathrm{mM}$ imidazole in lysis buffer. Fractions containing the purest yCGL (as determined by SDS-PAGE) were pooled, concentrated, and dialyzed overnight against storage buffer. Glycerol was added to $20 \%(\mathrm{v} / \mathrm{v})$, and $0.5 \mathrm{~mL}$ aliquots of the protein were flash-frozen on dry ice and stored at $-80^{\circ} \mathrm{C}$. 
2.3. Determination of steady-state kinetic parameters for hydrolysis of L-Cth, L-Cys, L$O A S$, and L-Hcys.

Enzyme activity was measured in a total reaction volume of $100 \mu \mathrm{L}$ at $25^{\circ} \mathrm{C}$ and detected on a Spectramax 340 microtiter plate spectrophotometer (Molecular Devices). The assay buffer was composed of $50 \mathrm{mM}$ potassium phosphate, $\mathrm{pH} 7.2$, with $20 \mu \mathrm{M}$ PLP. Reaction of DTNB $\left(\varepsilon_{412}=13600 \mathrm{M}^{-1} \mathrm{~cm}^{-1}\right)$ with the free thiol group of the product of L-Cth hydrolysis was monitored at $412 \mathrm{~nm}$ (Ellman, 1959; Yamagata et al., 1993; Figure 2.1). The amount of yCGL enzyme added ranged between $0.45-19.5 \mu \mathrm{M}$, depending on the activity of the specific site-directed variant. The $\gamma$-elimination of LHcys was detected via a coupled assay with $34.5 \mu \mathrm{M}$ HO-HxoDH, in which the reduction of the $\alpha$-ketobutyrate product, with concomitant oxidation of $\mathrm{NADH}\left(\varepsilon_{340}=6200 \mathrm{M}^{-1} \mathrm{~cm}^{-}\right.$ ${ }^{1}$ ), was monitored at $340 \mathrm{~nm}$ (Aitken et al., 2003; Figure 2.1). The $\beta$-elimination of L-Cys and L-OAS was detected by a coupled assay with $3.5 \mu \mathrm{M} \mathrm{LDH}$, in which the reduction of the pyruvate product, with concomitant oxidation of $\mathrm{NADH}$, was monitored at $340 \mathrm{~nm}$ (Lodha and Aitken, 2011). The amount of wild-type or site-directed variant of yCGL added ranged from $2.25-9.75 \mu \mathrm{M}$, depending on the activity of the specific enzyme. A background reading was taken in all cases before the reaction was initiated by the addition of enzyme. Data were fit to the Michaelis-Menten equation (1) to obtain values of $k_{\text {cat }}$ and $K_{\mathrm{m}}$ for the hydrolysis of L-Cth, L-OAS, and L-Hcys, and $k_{\text {cat }} / K_{\mathrm{m}}^{\text {L-Cth }}{ }^{\text {was }}$ obtained independently from equation 2 . The L-Cys hydrolysis data were fit to equation 3, which modifies the Michaelis-Menten equation to incorporate the $K_{\mathrm{i}}^{L-C y s}$ term for substrate inhibition by L-Cys, and $k_{\text {cat }} / K_{\mathrm{m}}$ values were obtained independently from equation 4. Data were fit by nonlinear regression with Kaleidagraph 4.0 (Synergy 
Software). For site-directed variants with activity so low that saturation was not observed, $k_{\text {cat }} / K_{\mathrm{m}}$ values were obtained by linear regression, based on the assumption that $K_{\mathrm{m}} \gg$ [substrate], such that the denominator of the Michaelis-Menten equation $\left(K_{\mathrm{m}}+\right.$ [substrate]) approximates $K_{\mathrm{m}}$ and the slope of the linear fit provides an estimate of catalytic efficiency (equation 5).

$$
\begin{gathered}
\frac{v}{[E]}=\frac{k_{c a t} \times[S]}{K_{m}+[S]} \\
\frac{v}{[E]}=\frac{k_{c a t} / K_{m} \times[S]}{1+[S] / K_{m}} \\
\frac{v}{[E]}=\frac{k_{c a t} \times[S]}{K_{m}+[S]\left(1+[S] / K_{i}{ }^{L-c y s}\right)} \\
\frac{v}{[E]}=\frac{k_{c a t} / K_{m} \times[S]}{1+[S] / K_{m}\left(1+[S] / K_{i}{ }^{L-c y s}\right)} \\
\frac{v}{[E]}=k_{c a t} / K_{m} \times[S]
\end{gathered}
$$


2.4. Evaluation of the $p H$ dependence of wild-type $y C G L$ and site-directed variants.

The $\mathrm{pH}$ dependence of the L-Cth hydrolysis activity of wild type and site-directed variants of yCGL was determined with the DTNB assay, which monitors the reaction of the free thiol product of L-Cth hydrolysis with the DTNB reagent at $412 \mathrm{~nm}\left(\varepsilon_{412}=13\right.$ $\left.600 \mathrm{M}^{-1} \mathrm{~cm}^{-1}\right)$. Reactions were carried out between $\mathrm{pH} 6.4$ and 10 in a three-component buffer comprised of $50 \mathrm{mM}$ MOPS ( $\left.K_{\mathrm{a}}=7.2\right), 50 \mathrm{mM}$ bicine $\left(\mathrm{p} K_{\mathrm{a}}=8.3\right)$, and $50 \mathrm{mM}$ proline $\left(\mathrm{p} K_{\mathrm{a}}=10.7\right)($ Peracchi et al., 1996). Enzyme activity was measured in a $100 \mu \mathrm{L}$ total volume with buffer containing $20 \mu \mathrm{M}$ PLP, $2 \mathrm{mM}$ DTNB, $0.45-19.5 \mu \mathrm{M}$ wild type or variant $\mathrm{yCGL}$, depending on the activity of the specific enzyme, and $5.5 \mathrm{mM} \mathrm{L-Cth}$, for specific activity measurements, or $0.01-5.5 \mathrm{mM} \mathrm{L}-\mathrm{Cth}$, for determination of $k_{\text {cat }} / K_{\mathrm{m}}{ }^{\mathrm{L}}$ Cth. Activity was monitored at $25^{\circ} \mathrm{C}$ with a Spectramax 340 microtiter plate spectrophotometer (Molecular Devices). Specific activity versus $\mathrm{pH}$ measurements were carried out in triplicate to obtain an estimate of the experimental error associated with each $\mathrm{pH}$ point. The $k_{\mathrm{cat}} / K_{\mathrm{m}}$ versus $\mathrm{pH}$ data were fit to the bell-shaped curve defined by equation 6 , in which $k_{\text {cat }} / K_{\mathrm{m}}{ }^{\mathrm{max}}$ is the upper limit for $k_{\text {cat }} / K_{\mathrm{m}}$ at the $\mathrm{pH}$ optimum (Aitken $e t$ al., 2003).

$$
k_{c a t} /_{K_{m}}=\frac{k_{c a t} / K_{m}{ }^{\max }}{1+10^{p K_{a 1}-p H}+10^{p H-p K_{a 2}}}
$$




\subsection{Absorbance Spectroscopy.}

Spectra were recorded between $250-1100 \mathrm{~nm}$ with a model HP8453 spectrophotometer (Agilent) at $25^{\circ} \mathrm{C}$. To observe the formation of reaction intermediates, a solution of enzyme was incubated with either $100 \mathrm{mM} \mathrm{L-alanine} \mathrm{or} 3 \mathrm{mM} \mathrm{L-Cth}$ in 50 $\mathrm{mM}$ phosphate, $\mathrm{pH} \mathrm{7.2,} \mathrm{and} \mathrm{monitored} \mathrm{for} 120 \mathrm{~min}$. The absorbance spectrum of $20 \mu \mathrm{M}$ enzyme was recorded prior to adding the substrate.

\subsection{Spectrophotometric Titration of Internal Aldimine.}

The internal aldimine of yCGL and eCBL was titrated following the methods of Eliot and Kirsch (2002) and Aitken and Kirsch (2003). The pH of a solution of $20 \mu \mathrm{M}$ enzyme in $5 \mathrm{mM}$ MOPS ( $K_{\mathrm{a}} 7.2, \mathrm{pH} 6.5$, containing $0.5 \mathrm{M} \mathrm{KCl}$ ) was increased by successive additions of $0.5 \mathrm{M}$ TAPS ( $K_{\mathrm{a}} 8.4, \mathrm{pH} 7.3$ ) below $\mathrm{pH} 7.2,0.5 \mathrm{M}$ AMPSO (pKa 9, $\mathrm{pH} 10.6)$ between $\mathrm{pH} 7.2-9.0,0.5 \mathrm{M}$ CAPS $\left(\mathrm{p} K_{\mathrm{a}} 10.4, \mathrm{pH} 11.6\right)$ between $\mathrm{pH} 9.0-$ 10.0 , and $10 \mathrm{M} \mathrm{NaOH}$ above $\mathrm{pH} 11$. The $\mathrm{pH}$ of the solution was determined before each measurement, and spectra were recorded between $250-500 \mathrm{~nm}$ on a model HP8453 spectrophotometer (Agilent) at $25^{\circ} \mathrm{C}$. The absorbance data obtained at 421 and $392 \mathrm{~nm}$ were fitted to equations 7 and 8 , respectively:

$$
\begin{aligned}
& A=\frac{A_{1}-A_{2}}{1+10^{(p H-p K a)}}+A_{2} \\
& A=\frac{A_{1}-A_{2}}{1+10^{(p K a-p H)}}+A_{2}
\end{aligned}
$$

where $A_{1}$ and $A_{2}$ are the high and low absorbance limits at a particular wavelength, respectively. 


\section{RESULTS}

\subsection{Construction, expression, and purification of the site-directed variants.}

Site-directed mutants were constructed by overlap-extension PCR. The 5' and 3' segments, produced in the first round of PCR, (Figure 3.1A) were reassembled and the resulting full-length amplicons (Figure 3.1B) were introduced at the $\mathrm{NdeI} / \mathrm{SaCI}$ sites of the pTrc99aAF vector, expressed in $E$. coli strain ER1821 $\triangle \mathrm{CBL}$ and the resulting protein was purified by Ni-NTA affinity chromatography. All site-directed variants were soluble with yields between $1.62-5.98 \mathrm{mg} / \mathrm{L}$ (Table 3.1 ), similar to the $1.04 \mathrm{mg} / \mathrm{mL}$ yield of the N-terminally His-tagged wild-type yCGL. The purified wild-type yCGL and site-directed variants were estimated to be $\geq 90 \%$ pure as judged by SDS-PAGE analysis (Figure 3.2).

\subsection{The wild-type yCGL enzyme.}

The activity of wild-type and site-directed variants of yCGL was measured using the DTNB and HO-HxoDH assays, which detect the presence of the L-cysteine or LHcys, and $\alpha$-ketobutyrate or pyruvate, products, respectively, of L-Cth hydrolysis (Figure 1.10A). These assays detect L-Cth hydrolysis and are not specific for $\beta$ - or $\gamma$-elimination. Comparison of the kinetic parameters of site-directed variants for the physiological substate provides insight on their role in substrate binding and catalysis. The alternative substrates L-Cys and L-OAS, which can only be hydrolyzed via a $\beta$-elimination mechanism, and L-Hcys, which is $\gamma$-elimination-specific (Figure 1.10B), enable investigation of reaction specificity. Wild-type yCGL catalyzes the hydrolysis of L-Cth 


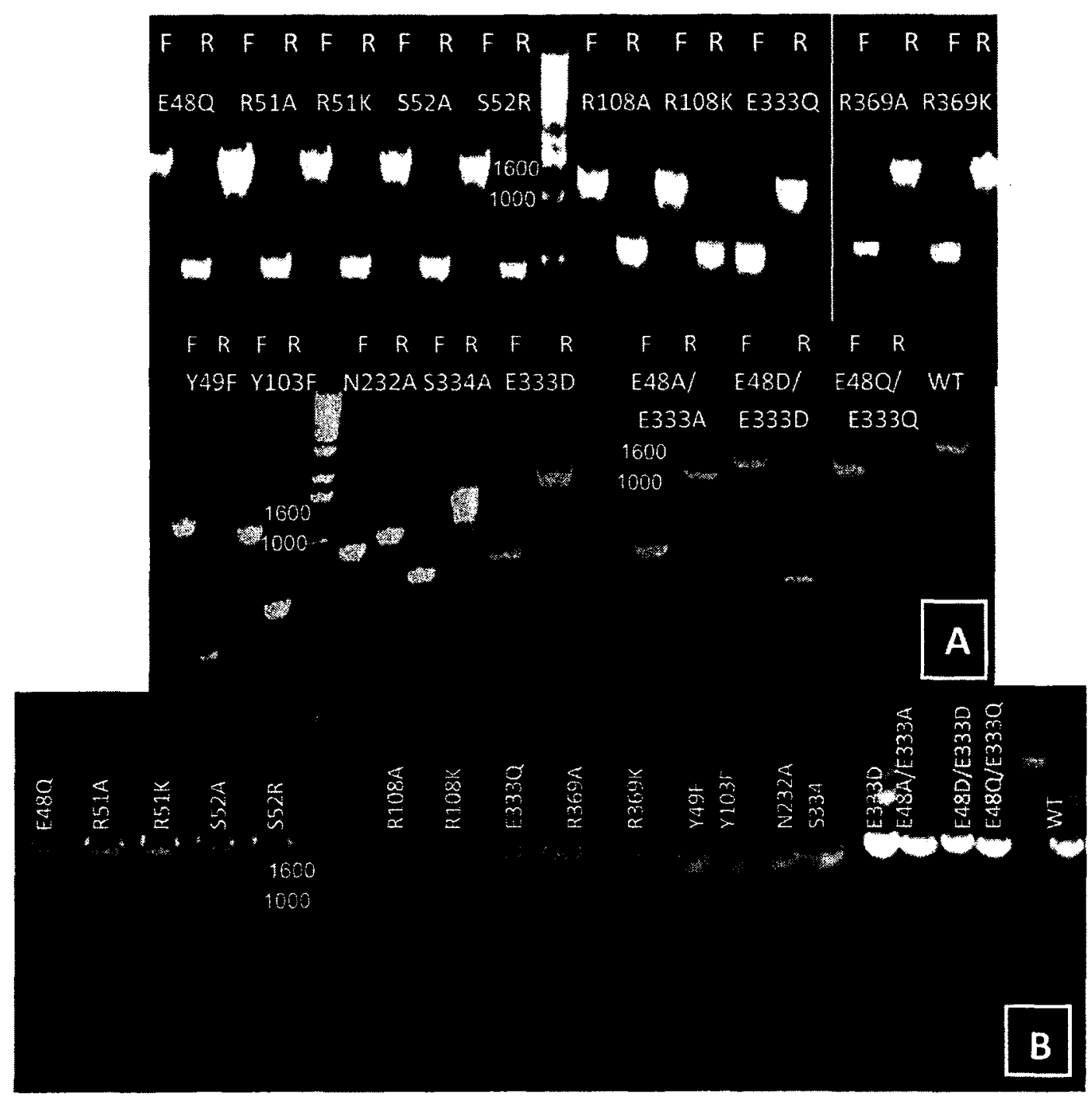

Figure 3.1. Amplicons of the first and second rounds of overlap-extension PCR for construction of site-directed mutants. (A) Initial PCR to introduce desired mutation into yCGL gene, with one mutagenic and one flanking vector primer. Lanes labelled "F" are reactions which employed the 5' (forward) mutagenic primer and the pSECseq7r vector primer; lanes labelled " $R$ " are reactions which employed the 3' (reverse-complement) mutagenic and pSECseq0 vector primers to generate the overlapping 3' and 5' segments, respectively. (B) Reassembly PCR using the pSECseq0 and pSECseq7r flanking primers to combine the $3^{\prime}$ and 5' coding sequence segments, producing the full-length sitedirected mutants of yCGL. "WT" indicates the positive control, where only the flanking primers were used to amplify wild-type yCGL template. Sizes of the molecular weight ladder bands are indicated in base pairs. 
Table 3.1. Yield of yCGL site-directed variants.

\begin{tabular}{|c|c|}
\hline yCGL variant & Yield (mg/L) \\
\hline yCGL & 1.04 \\
\hline E48A & n.a. $^{{ }^{2}}$ \\
\hline E48D & n.a. $^{{ }^{2}}$ \\
\hline E48Q & 5.98 \\
\hline Y49F & 3.82 \\
\hline R51A & 1.62 \\
\hline R51K & 4.91 \\
\hline S52A & 3.82 \\
\hline S52R & 5.81 \\
\hline Y103F & 3.78 \\
\hline R108A & 5.32 \\
\hline R108K & 3.68 \\
\hline N232A & 3.72 \\
\hline E333A & n.a. \\
\hline E333D & 3.29 \\
\hline E333Q & 3.86 \\
\hline E48A/E333A & 3.97 \\
\hline E48D/E333D & 4.79 \\
\hline E48Q/E333Q & 2.68 \\
\hline S334A & 5.24 \\
\hline R369A & 4.04 \\
\hline R369K & 5.24 \\
\hline
\end{tabular}

${ }^{\mathrm{a}}$ Variant purified by Farsi et al. (2009) and yield not reported. 


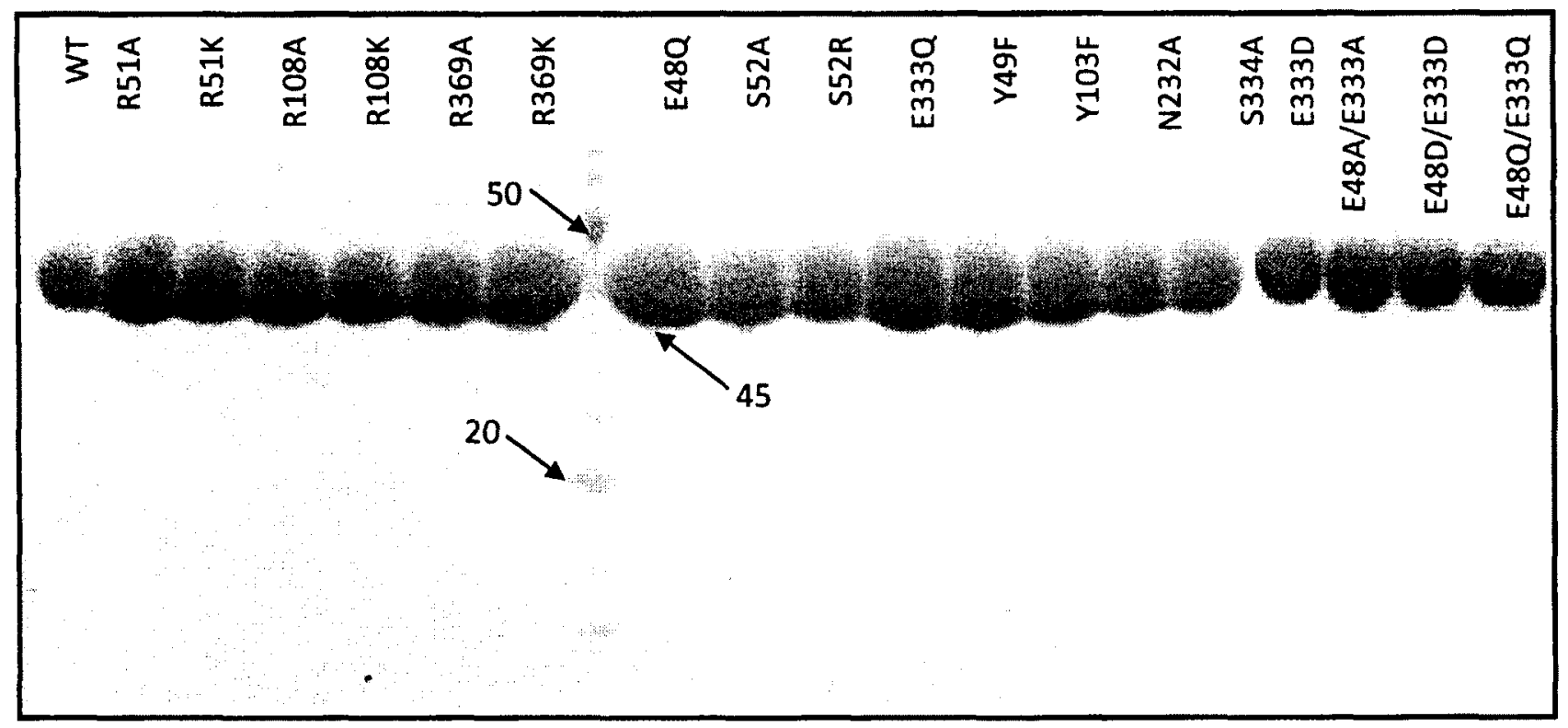

Figure 3.2. SDS-PAGE demonstrating the purity of $5 \mu \mathrm{g}$ of wild-type and site-directed variants of yCGL. Approximate sizes of bands are indicated in $\mathrm{kDa}$; the molecular weight of $\mathrm{yCGL}$ with the $\mathrm{N}$-terminal affinity tag is $45.1 \mathrm{kDa}$. 
with a catalytic efficiency $\left(k_{\text {cat }} / K_{\mathrm{m}}^{\text {L-Cth }}\right)$ of $(2.1 \pm 0.1) \times 10^{3} \mathrm{M}^{-1} \mathrm{~s}^{-1}$ (Table 3.2). The catalytic efficiency of the wild-type yCGL enzyme for the $\beta$-elimination of L-Cys is only 1.2-fold lower than that for the hydrolysis of physiological substrate, L-Cth (Table 3.2). The wild-type yCGL also displays substrate inhibition by L-Cys (Figure 3.3A; Table 3.2; $K_{\mathrm{i}}^{\mathrm{L}-\mathrm{Cys}}=2 \mathrm{mM}$ ), that is not observed for L-Cth. In contrast, the catalytic efficiency of yCGL for the $\beta$-elimination of L-OAS is 560 -fold lower than that for L-Cth, primarily due to the 155 -fold lower turnover $\left(k_{\text {cat }}\right)$ of the former, as the $K_{\mathrm{m}}{ }^{\mathrm{L}-\mathrm{OAS}}$ is only 3 -fold higher than $K_{\mathrm{m}}{ }^{\mathrm{L}-\mathrm{Cth}}$ (Table 3.2). The wild-type enzyme is not saturated by L-Hcys between 0.4 $100 \mathrm{mM}$ (Figure 3.3B; Table 3.2). Therefore, $k_{\mathrm{cat}} / K_{\mathrm{m}}^{\text {L-Hcys }}$ was determined via linear regression, based on the assumption that $K_{\mathrm{m}}^{\text {L-cys }}>$ [L-Hcys] (equation 5). The catalytic efficiency for the $\gamma$-elimination of L-Hcys is 77,000 -fold lower than that for L-Cth hydrolysis (Table 3.2).

The $\mathrm{pH}$ dependence of L-Cth hydrolysis of the wild-type yCGL enzyme and sitedirected variants were examined in this study to investigate the potential roles of activesite residues in proton transfer or maintenance of charge distribution. The bell-shaped $\mathrm{pH}$ dependence of activity, common to many enzymes, is a function of the ionization of the substrate and kinetically influential residues (Marangoni, 2003). While many groups in the enzyme will titrate across the $\mathrm{pH}$ range tested, only those that influence the probe, in this case activity, are reflected in the $\mathrm{p} K_{\mathrm{a}}$ values of the acidic and basic limbs of the $\mathrm{pH}$ profile. The titrating groups responsible for the $\mathrm{p} K_{\mathrm{a}}$ values of the $\mathrm{pH}$ profile must be in the appropriate protonation state to allow efficient substrate binding and/or catalysis. If substitution of a residue results in the elimination of either the acidic or basic limbs of the $\mathrm{pH}$ profile, this suggests that the targeted residue is the titrating group. Thus $\mathrm{pH}$ profiles, 
Table 3.2. Kinetic parameters for the hydrolysis of L-Cth, L-Cys, L-OAS, and L-Hcys by wild-type yCGL.

\begin{tabular}{|c|c|c|c|c|}
\hline Assay & $k_{\mathrm{cat}}\left(\mathrm{s}^{-1}\right)$ & $K_{\mathrm{m}}(\mathrm{mM})$ & $K_{i}^{\text {L-Cys }}(\mathrm{mM})$ & $k_{\text {cat }} / K_{\mathrm{m}}\left(\mathrm{M}^{-1} \mathrm{~s}^{-1}\right)$ \\
\hline \multirow{2}{*}{ DTNB } & \multicolumn{4}{|c|}{ L-Cth $\rightarrow$ L-Cys + a-ketobutyrate $+\mathrm{NH}_{3}$} \\
\hline & $1.51 \pm 0.03$ & $0.71 \pm 0.04$ & & $(2.1 \pm 0.1) \times 10^{3}$ \\
\hline \multirow{2}{*}{ LDH } & \multicolumn{4}{|c|}{ L-Cys $\rightarrow$ pyruvate $+\mathrm{H}_{2} \mathrm{~S}+\mathrm{NH}_{3}$} \\
\hline & $0.15 \pm 0.01$ & $0.09 \pm 0.01$ & $2 \pm 0.3$ & $(1.7 \pm 0.2) \times 10^{3}$ \\
\hline \multirow{2}{*}{ LDH } & \multicolumn{4}{|c|}{ L-OAS $\rightarrow$ acetate + pyruvate $+\mathrm{NH}_{3}$} \\
\hline & $0.0097 \pm 0.0002$ & $2.5 \pm 0.3$ & & $3.8 \pm 0.4$ \\
\hline \multirow{2}{*}{ HO-HxoDH } & \multicolumn{4}{|c|}{ L-Hcys $\rightarrow \alpha$-ketobutyrate $+\mathrm{H}_{2} \mathrm{~S}+\mathrm{NH}_{3}$} \\
\hline & n.s. ${ }^{\mathrm{a}}$ & n.s. & & $0.027 \pm 0.005$ \\
\hline
\end{tabular}

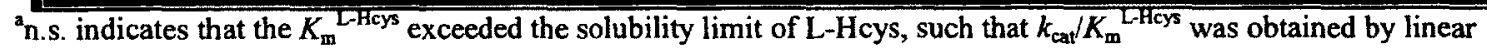
regression. 

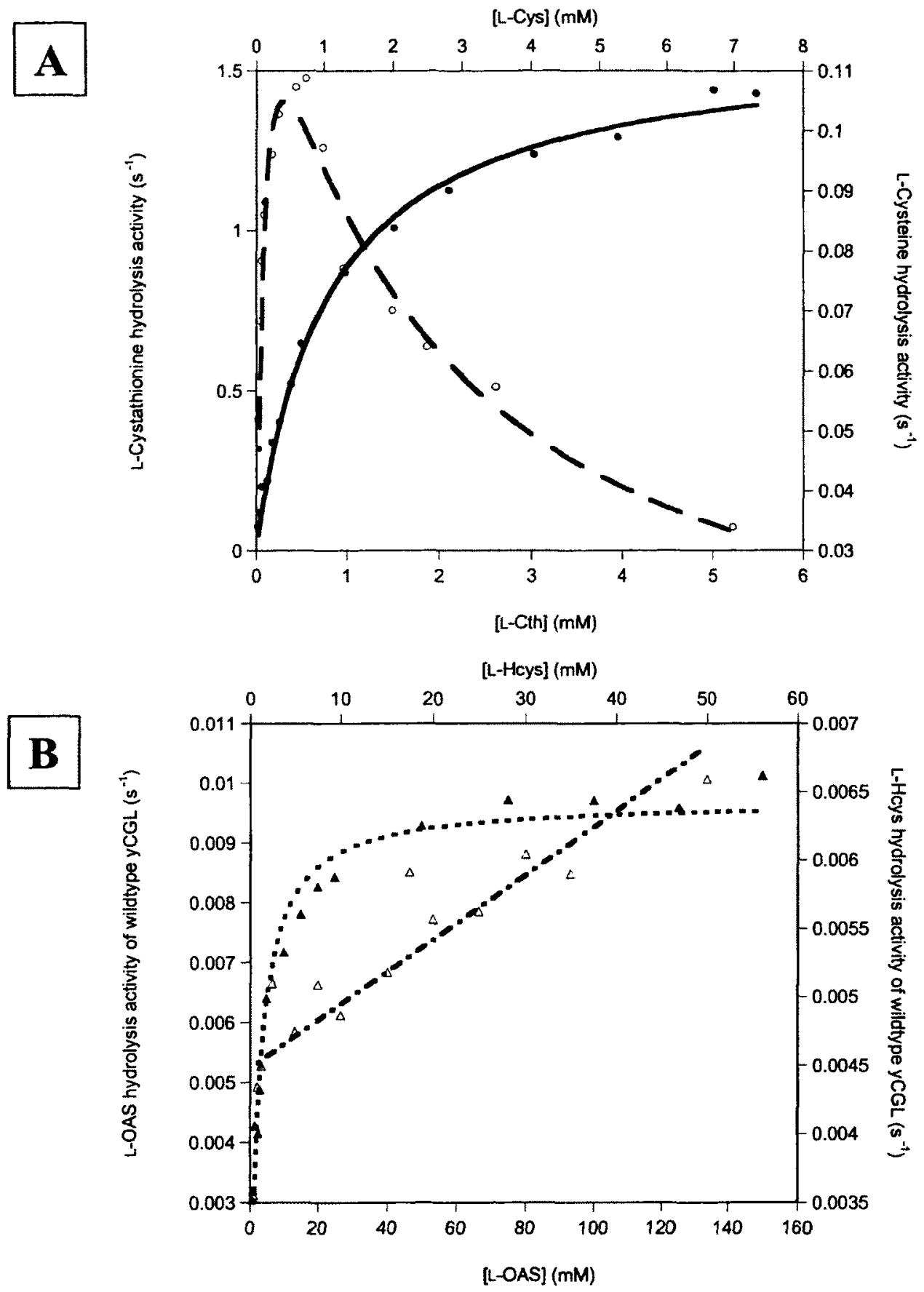

Figure 3.3. The substrate and reaction specificity of wild-type yCGL. (A) Dependence of the yCGL-catalyzed hydrolysis of L-Cth (solid circle) and L-Cys (empty circle) on substrate concentration. (B) Dependence of the wild-type yCGL-catayzed hydrolysis of L-OAS (solid triangle) and L-Hcys (empty triangle) on substrate concentration. The L-Cth (solid line) and L-OAS (dotted line) data were fit to the Michaelis-Menten equation (equation 1), and the L-Cys (dashed line) data were fit to equation 3, which incorporates the $K_{\mathrm{i}}^{\mathrm{L}-\mathrm{Cys}}$ term for substrate inhibition, and the L-Hcys data (mixed dotted/dashed line) were fit by linear regression. 
in combination with site-directed mutagenesis, provide another tool by which to explore the roles of active-site residues. In this study, the $\mathrm{pH}$ dependence of specific activity was determined for all site-directed variants (Table 3.3), as a screen for substitutions modifying the $\mathrm{pH}$ profile of $\mathrm{yCGL}$, as a specific activity $\mathrm{pH}$ profile provides only an estimate of the $\mathrm{pH}$ optimum and possible shifts in $\mathrm{p} K_{\mathrm{a}}$ values. Those variants which displayed a shift in $\mathrm{pH}$ dependence were subsequently investigated further by characterizing their $k_{\text {cat }} / K_{\mathrm{m}}{ }^{\mathrm{L} \text {-Cth }}$ versus $\mathrm{pH}$ profiles.

The $\mathrm{pH}$ dependence of $k_{\text {cat }} / K_{\mathrm{m}}{ }^{\mathrm{L}-\mathrm{Cth}}$ of yCGL is bell-shaped, with an optimum of $7.6-8$ (Figure 3.4). The $\mathrm{p} K_{\mathrm{a}}$ values of the acidic and basic limbs of the $k_{\text {cat }} / K_{\mathrm{m}}{ }^{L-C \text { th }}$ versus $\mathrm{pH}$ profile are $8.1 \pm 0.4$ and $7.1 \pm 0.4$, respectively (Table 3.4 ). The $\mathrm{p} K_{\mathrm{a}}$ of the internal aldimine of wild-type yCGL is $8.84 \pm 0.07$, as determined by spectrophotometric titration, which is approximately $2 \mathrm{pH}$ units lower than that of the wild-type eCBL internal aldimine, which has a $K_{\mathrm{a}}$ of $10.81 \pm 0.08$ (Figure 3.5).

Following 120 -min incubation $100 \mathrm{mM}$ L-alanine, which is not a substrate for $\beta$ or $\gamma$-elimination or substitution, but can undergo transamination or racemization (Figure 1.11), the 420-nm absorbance of the wild-type yCGL enzyme, characteristic of the internal aldimine form of the PLP cofactor, is decreased accompanied by a gradual increase in absorbance between 300-350 nm (Figure 3.6A). In contrast, upon reaction with $3 \mathrm{mM} \mathrm{L-Cth}$, the 420-nm peak of the wild-type yCGL enzyme decreases in intensity, with a concomitant increase in absorbance forming a shoulder at $330 \mathrm{~nm}$ (Figure 3.6B). 
Table 3.3. Parameters determined from specific activity versus $\mathrm{pH}$ profiles of $\mathrm{yCGL}$ and site-directed variants.

\begin{tabular}{|c|c|c|c|}
\hline Enzyme & pH optimum & $\mathbf{p} \boldsymbol{K}_{a 1}$ & $\mathrm{p} K_{a 2}$ \\
\hline yCGL & $7.2-7.8$ & $6.77 \pm 0.05$ & $8.84 \pm 0.06$ \\
\hline E48A & $8.2-9.0$ & $7.32 \pm 0.06$ & $9.4 \pm 0.1$ \\
\hline E48D & $7.2-7.8$ & $6.57 \pm 0.06$ & $8.56 \pm 0.06$ \\
\hline E48Q & $8.0-8.6$ & $7.05 \pm 0.05$ & $9.19 \pm 0.06$ \\
\hline Y49F & $7.4-8.0$ & $7.09 \pm 0.05$ & $8.23 \pm 0.05$ \\
\hline R51A & $7.0-8.0$ & $6.4 \pm 0.1$ & $8.8 \pm 0.1$ \\
\hline R51K & $7.4-8.0$ & $7.13 \pm 0.04$ & $8.14 \pm 0.04$ \\
\hline S52A & $7.6-8.2$ & $7.44 \pm 0.07$ & $8.51 \pm 0.08$ \\
\hline S52R & $7.4-8.0$ & $7.6 \pm 0.1$ & $7.8 \pm 0.1$ \\
\hline Y103F & $8.4-9.0$ & $6.66 \pm 0.06$ & $9.91 \pm 0.09$ \\
\hline R108A & $7.8-8.2$ & $7.16 \pm 0.03$ & $8.67 \pm 0.03$ \\
\hline R108K & $7.6-8.2$ & $7.50 \pm 0.06$ & $8.10 \pm 0.07$ \\
\hline $\mathbf{N} 232 A$ & $7.6-8.2$ & $7.49 \pm 0.04$ & $8.25 \pm 0.05$ \\
\hline E333A & $8.2-8.6$ & $8.1 \pm 0.1$ & $8.8 \pm 0.1$ \\
\hline E333D & $7.4-8.0$ & $6.9 \pm 0.6$ & $8.5 \pm 0.6$ \\
\hline $\mathbf{E 3 3 3 Q}$ & $8.2-8.6$ & $8.37 \pm 0.06$ & $8.46 \pm 0.07$ \\
\hline E48A/E333A & $8.4-9.4$ & $9.2 \pm 0.3$ & $8.1 \pm 0.3$ \\
\hline E48D/E333D & $7.6-8.2$ & $7.3 \pm 0.2$ & $8.4 \pm 0.2$ \\
\hline E48Q/E333Q & $8.2-8.8$ & $8.6 \pm 0.3$ & $8.1 \pm 0.3$ \\
\hline S334A & $7.4-8.0$ & $7.1 \pm 0.1$ & $8.6 \pm 0.1$ \\
\hline
\end{tabular}




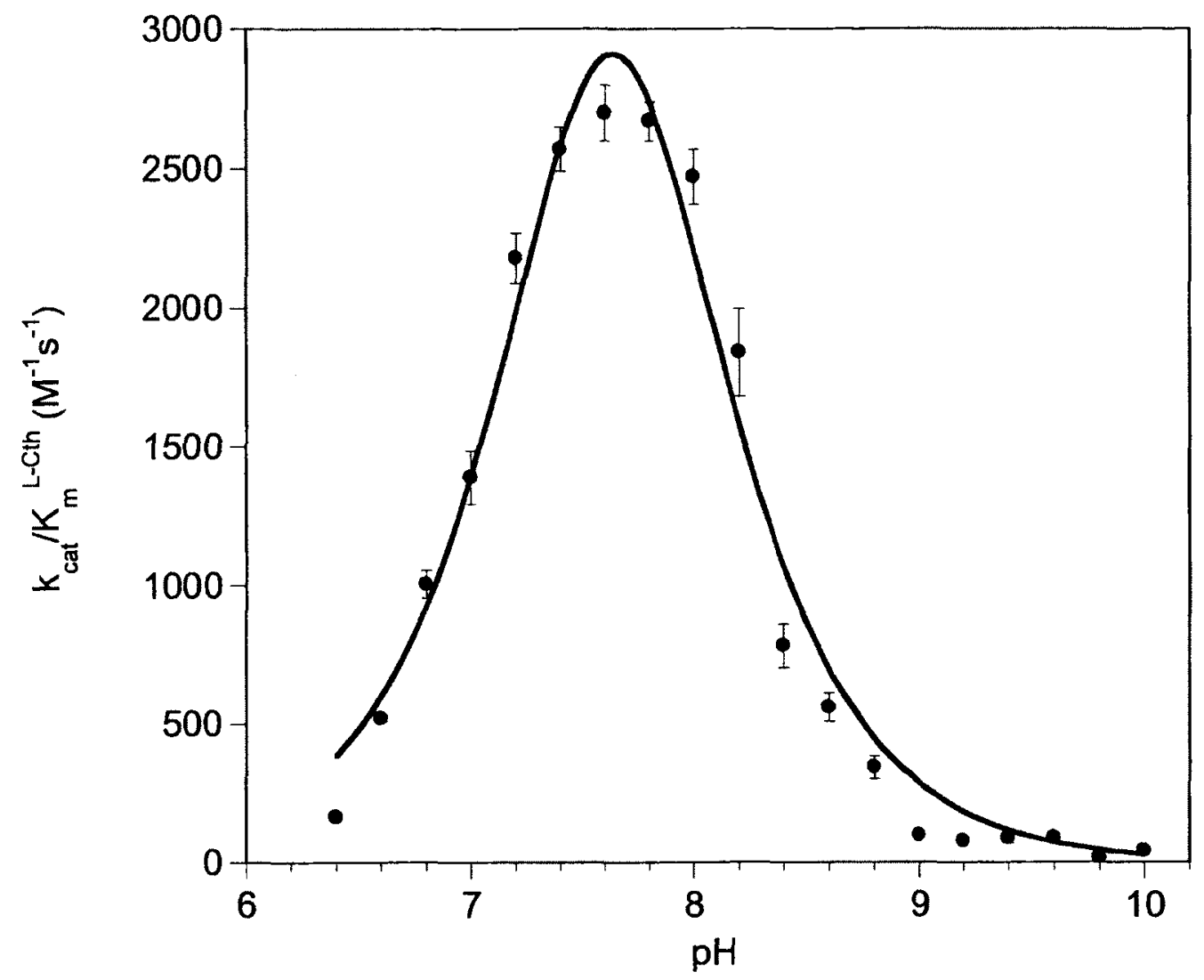

Figure 3.4. The pH dependence of $k_{\text {cat }} / K_{\mathrm{m}}{ }^{\text {L-Cth }}$ for wild-type yCGL. 
Table 3.4. Parameters determined from $k_{\text {cat }} / K_{\mathrm{m}}^{\text {L-Cth }}$ versus $\mathrm{pH}$ profiles of yCGL and sitedirected variants.

\begin{tabular}{|l|c|c|}
\hline Enzyme & $\mathrm{p} \boldsymbol{K}_{a 1}$ & $\mathrm{p} \boldsymbol{K}_{a 2}$ \\
\hline yCGL & $8.1 \pm 0.4$ & $7.1 \pm 0.4$ \\
\hline E48A & $7.4 \pm 0.4$ & $8.5 \pm 0.4$ \\
\hline E48D & $7.3 \pm 0.2$ & $7.7 \pm 0.2$ \\
\hline E48Q & $7.5 \pm 0.1$ & $8.1 \pm 0.1$ \\
\hline Y103F & $7.9 \pm 0.3$ & $7.7 \pm 0.3$ \\
\hline E333A & $9 \pm 1$ & $7 \pm 1$ \\
\hline E333D & $8 \pm 1$ & $7 \pm 1$ \\
\hline E333Q & $8.7 \pm 0.5$ & $7.5 \pm 0.5$ \\
\hline E48A/E333A & $9 \pm 1$ & $8 \pm 1$ \\
\hline E48D/E333D & $7.6 \pm 0.1$ & $8.3 \pm 0.1$ \\
\hline E48Q/E333Q & $9.2 \pm 0.8$ & $7.6 \pm 0.8$ \\
\hline
\end{tabular}



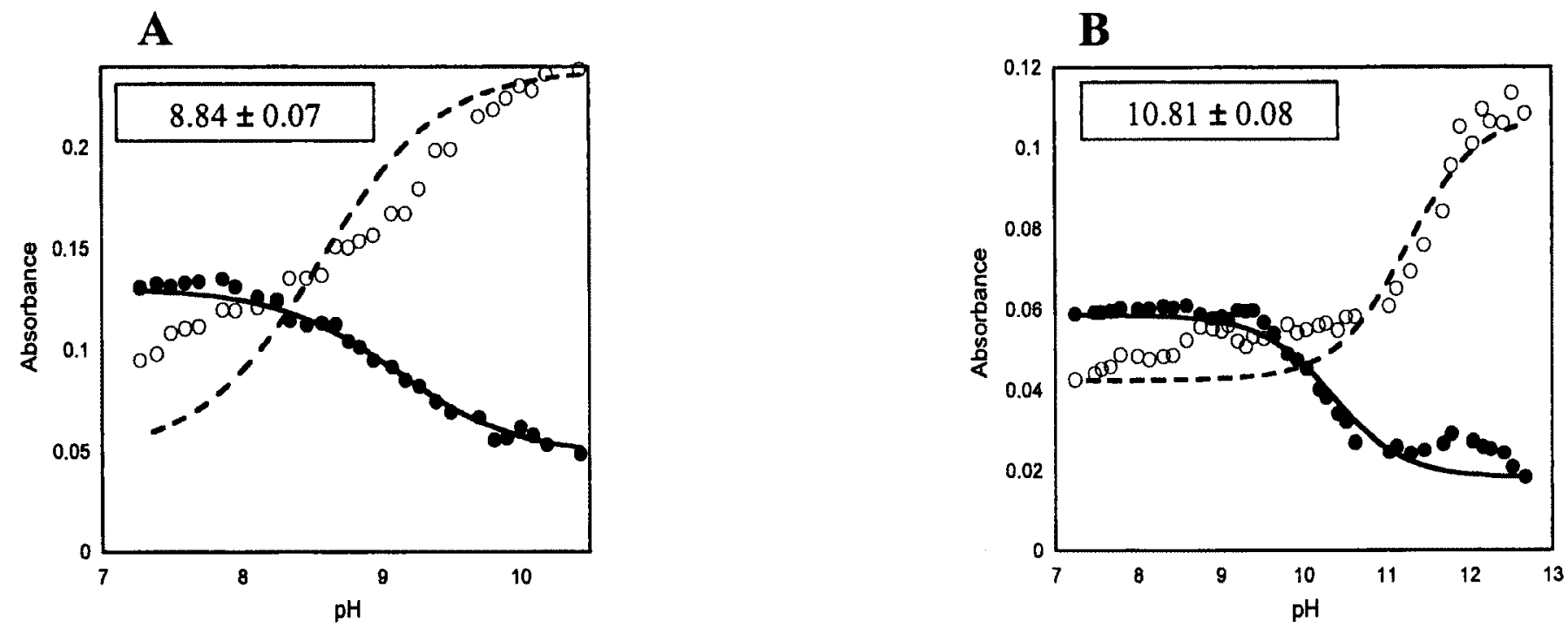

Figure 3.5. Spectrophotometric titration of (A) yCGL and (B) eCBL, showing dependence on $\mathrm{pH}$ of absorbance at $421 \mathrm{~nm}$ (solid circle) and $390 \mathrm{~nm}$ (empty circle). The $421 \mathrm{~nm}$ and $390 \mathrm{~nm}$ data were fit to equations 7 and 8 (solid and dashed lines, respectively) to give the indicated $\mathrm{p} K_{\mathrm{a}}$ values of the internal aldimine. 

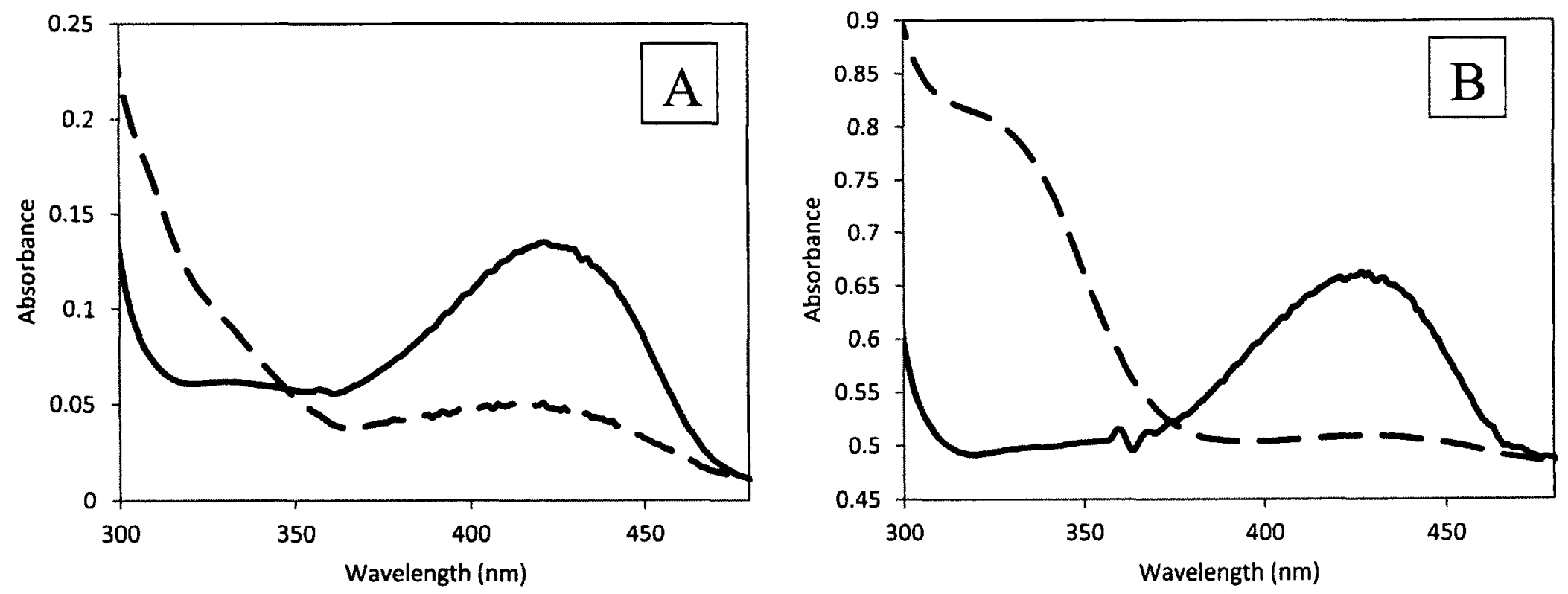

Figure 3.6. Absorbance spectrum of PLP cofactor of wild-type yCGL before (solid line) and after (dashed line) incubation for 120 min with (A) $100 \mathrm{mM} \mathrm{L}$-alanine or (B) $3 \mathrm{mM} \mathrm{L-Cth}$. 


\subsection{The $R 51 A / K, S 52 A / R, R 108 A / K, N 232 A$, and $R 369 A / K$ variants.}

The $k_{\text {cat }} / K_{\mathrm{m}}{ }^{\mathrm{L}-\mathrm{Cth}}$ values of R51A and R51K are decreased $\sim 38,000$-fold and 340 fold, respectively, compared to the wild-type enzyme (Table 3.5). Estimates of $k_{\text {cat }}$ and $K_{\mathrm{m}}^{\text {L-Cth }}$ could not be determined for these variants because they are not saturated within the solubility limit of L-Cth. The hydrolysis of L-Cys and L-Hcys is not detectable for either variant and although L-OAS hydrolysis was detected for R51K, the catalytic efficiency of this reaction is reduced by $\sim 420$-fold, compared to the wild-type enzyme (Table 3.5). Replacement of residue R51 with alanine or lysine results in minor shifts in the $\mathrm{pH}$ optimum of specific activity to $7.0-8.0$ and $7.4-8.0$, respectively (Table 3.3; Figure 3.7). Interestingly, incubation of the $\mathrm{R} 51 \mathrm{~A} / \mathrm{K}$ variants for $120 \mathrm{~min}$ with $100 \mathrm{mM}$ L-alanine results in the appearance of a $330-\mathrm{nm}$ peak in the absorbance spectrum of these enzymes (Figure 3.8A), a species not observed for the wild-type enzyme. In contrast, the effect of incubation with $3 \mathrm{mM} \mathrm{L}-\mathrm{Cth}$ on the absorbance spectra of the R51A/K variants is similar to wild-type yCGL (Figure 3.8B).

Substitution of S52 with alanine or arginine increases $K_{\mathrm{m}}^{\text {L-Cth }}$ by 5.6 -fold and 8fold, respectively, while $k_{\text {cat }}$ is altered by less than 2-fold (Table 3.5). The 7-11-fold decreases in the catalytic efficiency of L-Cys hydrolysis of S52A and S52R are

dominated by the $\sim 18$-fold increase in $K_{\mathrm{m}}{ }^{\text {L-Cys }}$ observed for both variants (Table 3.5 ). In contrast, the kinetic parameters of the S52A/R variants for L-OAS hydrolysis are within 2.5-fold of the wild type enzyme (Table 3.5). The catalytic efficiency of the S52Rcatalyzed hydrolysis of L-Hcys is within 1.5-fold of the wild type, but hydrolysis activity of S52A with the L-Hcys substrate is undetectable (Table 3.5). 
Table 3.5. Kinetic parameters of L-Cth, L-Cys, L-OAS and L-Hcys hydrolysis by yCGL and site-directed variants of residues R51, S52, R108, N232, and R369.

\begin{tabular}{|c|c|c|c|c|}
\hline Enzyme & $k_{\mathrm{cat}}\left(\mathrm{s}^{-1}\right)$ & $K_{\mathrm{m}}(\mathrm{mM})$ & $K_{i}^{\text {Lys }}(\mathbf{m M})$ & $k_{\mathrm{cat}} / K_{\mathrm{m}}\left(\mathrm{M}^{-1} \mathrm{~s}^{-1}\right)$ \\
\hline \multicolumn{5}{|c|}{ L-Cth $\rightarrow$ L-Cys $+a$-ketobutyrate $+\mathrm{NH}_{3}$} \\
\hline yCGL & $1.51 \pm 0.03$ & $0.71 \pm 0.04$ & & $(2.1 \pm 0.1) \times 10^{3}$ \\
\hline R51A & n.s. & n.s. & & $(6.4 \pm 0.3) \times 10^{-2}$ \\
\hline R51K & n.s. & n.s. & & $6.2 \pm 0.1$ \\
\hline S52A & $3.2 \pm 0.1$ & $4 \pm 0.2$ & & $(7.9 \pm 0.1) \times 10^{2}$ \\
\hline S52R & $1.8 \pm 0.1$ & $5.9 \pm 0.5$ & & $(3 \pm 0.1) \times 10^{2}$ \\
\hline R108A & $0.049 \pm 0.004$ & $5.6 \pm 0.7$ & & $8.8 \pm 0.5$ \\
\hline R108K & $0.10 \pm 0.01$ & $9.2 \pm 1.8$ & & $10.5 \pm 0.1$ \\
\hline N232A & $0.46 \pm 0.08$ & $15 \pm 6$ & & $30 \pm 2$ \\
\hline R369A & n.s. & n.s. & & $0.12 \pm 0.02$ \\
\hline R369K & n.s. & n.s. & & $0.14 \pm 0.03$ \\
\hline \multicolumn{5}{|c|}{ L-Cys $\rightarrow$ pyruvate $+\mathrm{H}_{2} \mathrm{~S}+\mathrm{NH}_{3}$} \\
\hline yCGL & $0.15 \pm 0.01$ & $0.09 \pm 0.01$ & $2 \pm 0.3$ & $(1.7 \pm 0.2) \times 10^{3}$ \\
\hline S52A & $0.4 \pm 0.1$ & $1.6 \pm 0.6$ & $1 \pm 0.4$ & $(2.3 \pm 0.2) \times 10^{2}$ \\
\hline S52R & $0.25 \pm 0.06$ & $1.7 \pm 0.5$ & $1.4 \pm 0.5$ & $(1.5 \pm 0.1) \times 10^{2}$ \\
\hline R108A & $0.011 \pm 0.002$ & $0.21 \pm 0.08$ & $1.3 \pm 0.5$ & $(5 \pm 1) \times 10^{1}$ \\
\hline R108K & $0.04 \pm 0.02$ & $1 \pm 0.5$ & $0.4 \pm 0.2$ & $(4.3 \pm 0.5) \times 10^{1}$ \\
\hline N232A & $0.07 \pm 0.03$ & $1 \pm 0.6$ & $1.1 \pm 0.7$ & $(7 \pm 1) \times 10^{1}$ \\
\hline \multicolumn{5}{|c|}{ L-OAS $\rightarrow$ acetate + pyruvate $+\mathrm{NH}_{3}$} \\
\hline yCGL & $0.0097 \pm 0.0002$ & $2.5 \pm 0.3$ & & $3.8 \pm 0.4$ \\
\hline R51K & n.s. & n.s. & & $0.009 \pm 0.001$ \\
\hline S52A & $0.0069 \pm 0.0004$ & $2.9 \pm 0.7$ & & $2.3 \pm 0.5$ \\
\hline S52R & $0.0094 \pm 0.0006$ & $6 \pm 1$ & & $1.6 \pm 0.4$ \\
\hline R108A & $0.0056 \pm 0.0001$ & $1.9 \pm 0.2$ & & $2.9 \pm 0.2$ \\
\hline R108K & $0.0057 \pm 0.0001$ & $1.1 \pm 0.1$ & & $5.1 \pm 0.4$ \\
\hline N232A & n.s. & n.s. & & $0.014 \pm 0.003$ \\
\hline \multicolumn{5}{|c|}{ L-Hcys $\rightarrow$ a-ketobutyrate $+\mathrm{H}_{2} \mathrm{~S}+\mathrm{NH}_{3}$} \\
\hline yCGL & n.s. & n.s. & & $0.027 \pm 0.005$ \\
\hline S52R & n.s. & n.s. & & $0.039 \pm 0.008$ \\
\hline R108A & n.s. & n.s. & & $0.005 \pm 0.001$ \\
\hline
\end{tabular}

an.s. indicates that the $K_{\mathrm{m}}$ exceeded the solubility limit of the substrate, such that $k_{\text {cat }} / K_{\mathrm{m}}$ was obtained by linear regression. 

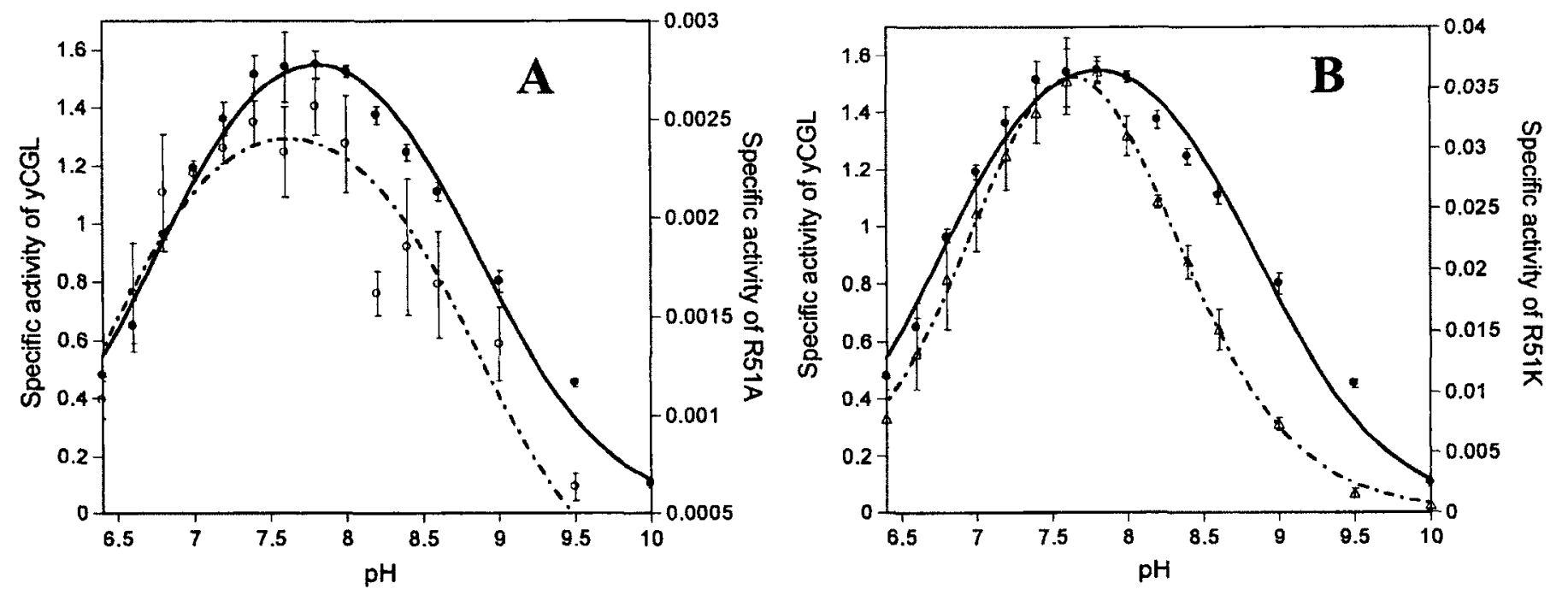

Figure 3.7. The pH dependence of specific activity of L-Cth hydrolysis of wild-type yCGL (solid circle, solid line) and the (A) R51A (empty circle, dashed line) and (B) R51K (triangle, dashed line) variants. 

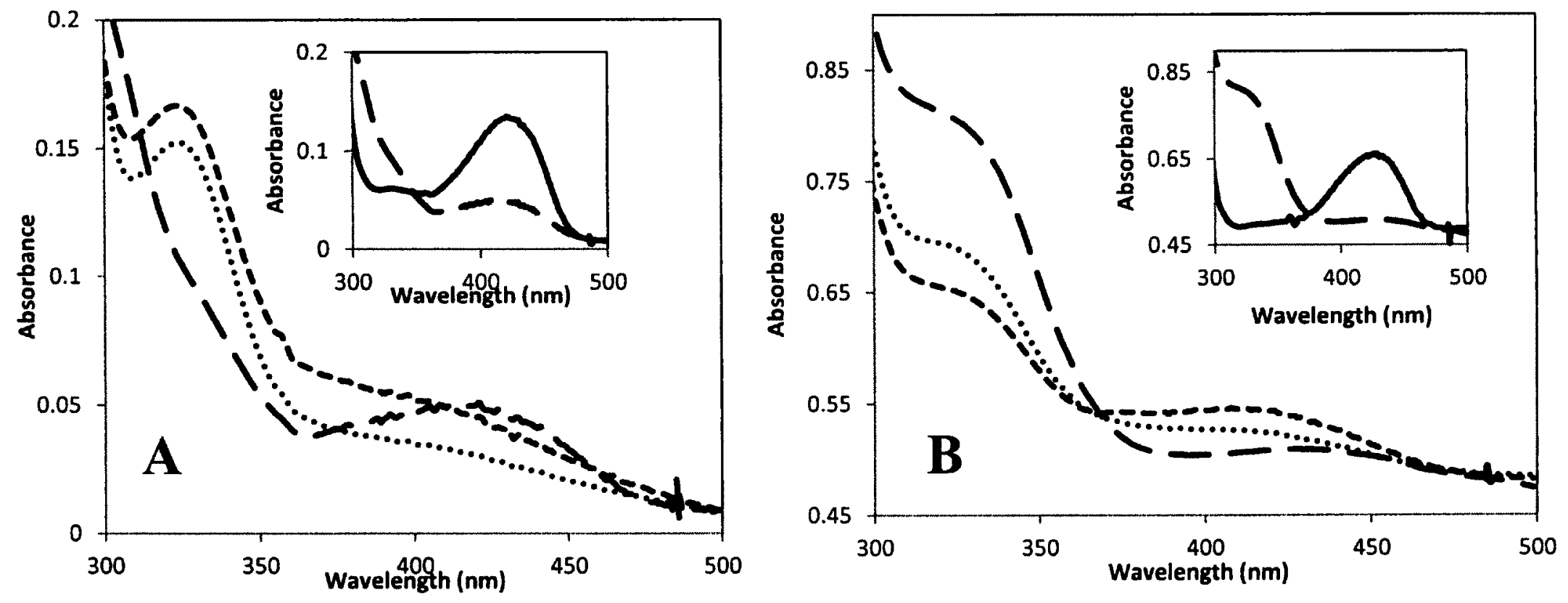

Figure 3.8. The effect of 120-min incubation with (A) $100 \mathrm{mM} \mathrm{L}$-alanine and (B) $3 \mathrm{mM} \mathrm{L}$-Cth on the absorbance spectra of the PLP cofactor of wild-type yCGL (long dashed line) and the R51A (short dashed line) and R51K (dotted line) variants. Insets: spectra of wild-type yCGL before (solid line) and after (long dashed line) 120-min incubation with substrate. 
The substitution of R108 with alanine and lysine results in 31 and 15-fold decreases in $k_{\text {cat }}$ and 8 and 13-fold increases in $K_{\mathrm{m}}^{\mathrm{L}-\mathrm{Cth}}$, respectively, compared to the wild-type enzyme (Table 3.5). The 34 and 40 -fold reductions in the catalytic efficiency for L-Cys hydrolysis of the R108A and R108K variants are dominated by the 13 and 4fold decreases in $k_{\text {cat }}$ (Table 3.5 ). The R108A/K variants lack detectable L-OAS hydrolysis activity and while L-Hcys hydrolysis by R108K is undetectable, the catalytic efficiency of this $\gamma$-elimination activity is reduced only 5-fold for R108A, compared to the wild type (Table 3.5). The specific activity versus $\mathrm{pH}$ profiles of the $\mathrm{R} 108 \mathrm{~A} / \mathrm{K}$ variants display shifts in $\mathrm{pH}$ optima to $7.8-8.2$, compared to $7.2-7.8$ for the wild-type enzyme (Figure 3.9; Table 3.3).

Substitution of N232 with alanine increases the $K_{\mathrm{m}}{ }^{\mathrm{L}-\mathrm{Cth}}$ by 21 -fold, compared to the wild-type enzyme (Table 3.5), and decreases the catalytic efficiency for the $\beta$ elimination of L-Cys by 24-fold, primarily as a result of the 11 -fold increase in $K_{\mathrm{m}}{ }^{\text {L-Cys }}$ (Table 3.5). Similar to R51K, the N232A variant cannot be saturated with L-OAS and a 270 -fold reduction in $k_{\text {cat }} / K_{\mathrm{m}}^{\text {L-OAS }}$ is observed (Table 3.5). The $\gamma$-elimination of L-Hcys is undetectable for the N232A variant (Table 3.5). The N232A replacement, as well as the alanine and arginine replacements of residues S52, results in minor increases in the $\mathrm{pH}$ optimum of specific activity to $\sim 7.6-8.2$ (Figure 3.10 ; Table 3.3 ).

The R369A and R369K variants cannot be saturated within the solubility limit of L-Cth and the $k_{\text {cat }} / K_{\mathrm{m}}{ }^{\text {L-Cth }}$ values of these enzymes are reduced 17,500 and 15,000 -fold, respectively, compared to the wild type (Table 3.5 ). The reduced activity of the alanine and lysine substitutions of $\mathrm{R} 369$ precluded investigation of the $\mathrm{pH}$ dependence of the 


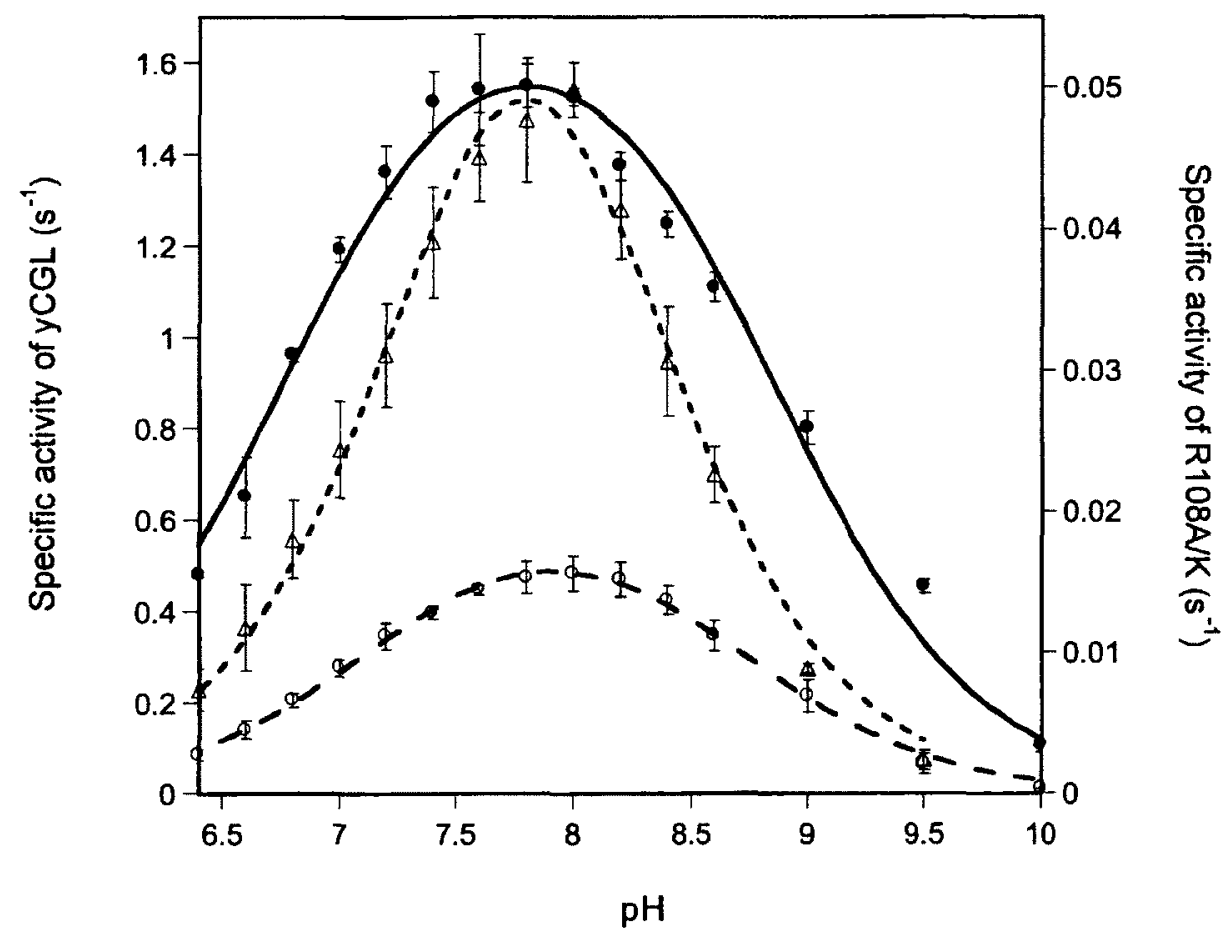

Figure 3.9. The $\mathrm{pH}$ dependence of specific activity of L-Cth hydrolysis of wild-type yCGL (solid circle) and the R108A (empty circle) and R108K (triangle) variants. 


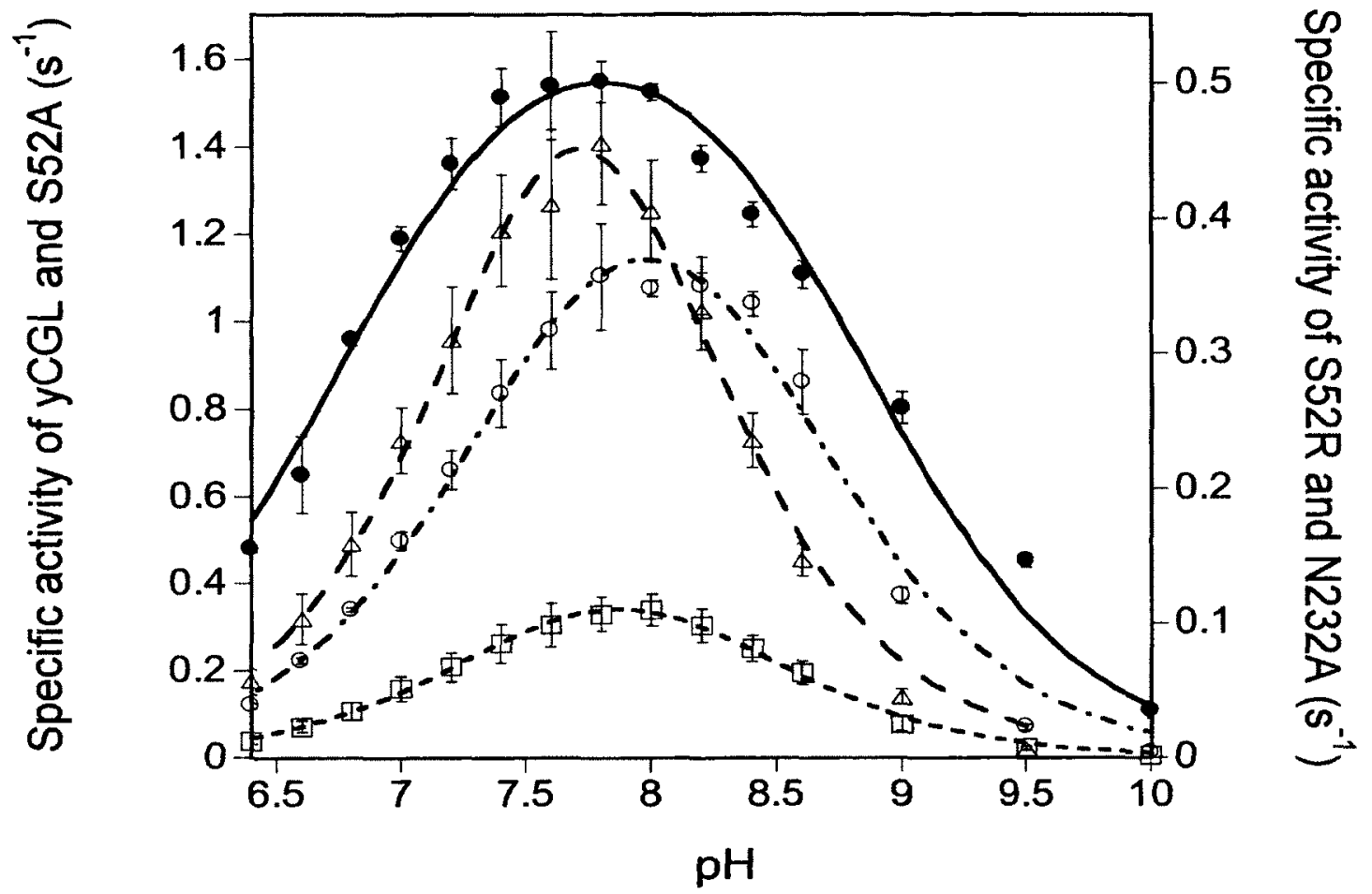

Figure 3.10. The $\mathrm{pH}$ dependence of specific activity of L-Cth hydrolysis of wild-type yCGL (solid circle) and the S52A (empty circle), S52R (triangle), and N232A (square) site-directed variants. 
specific activity of these variants. These variants also lack detectable activity for the hydrolysis of L-Cys, L-OAS, and L-Hcys.

\subsection{The $Y 49 F, Y 103 F$, and $S 334 A$ variants.}

The $k_{\text {cat }}$ for L-Cth hydrolysis by the Y $49 \mathrm{~F}$ variant is decreased $\sim 190$-fold from that of the wild-type enzyme, while the $K_{\mathrm{m}}{ }^{\mathrm{L}-\mathrm{Cth}}$ is increased only 4-fold (Table 3.6). Substitution of Y49 with phenylalanine results in a reduction of $k_{\text {cat }} / K_{\mathrm{m}}{ }^{\mathrm{L}}$-Cys by more than 20-fold, compared to wild-type yCGL, due to a reduction of similar magnitude in $k_{\text {cat }}$ (Table 3.6). In contrast, the kinetic parameters of Y49F-catalyzed L-OAS and L-Hcys hydrolysis are within 3-fold of the wild-type enzyme (Table 3.6). The $\mathrm{pH}$ optimum of the specific activity of $\mathrm{Y} 49 \mathrm{~F}$ is $7.4-8.0$, which represents a minor increase compared to the wild-type optimum of $7.2-7.8$ (Table 3.3 ; Figure $3.11 \mathrm{~A}$ ). The absorbance spectrum of yCGL-Y49F displays an increase in absorbance at $330 \mathrm{~nm}$, which is not observed for the wild-type enzyme, following reaction with L-alanine (Figure 3.12A, inset). However, the intensity of the $330-\mathrm{nm}$ band observed following incubation of the wild type with the physiological L-Cth substrate is reduced for the Y49F variant (Figure 3.12B).

Replacement of Y103 with phenylalanine reduces both the $k_{\text {cat }}$ and $K_{\mathrm{m}}{ }^{\mathrm{L}-\mathrm{Cth}}$ for hydrolysis of L-Cth by 420 -fold and 8-fold, respectively, compared to the wild type (Table 3.6). The 8-fold decrease in $K_{\mathrm{m}}^{\mathrm{L}-\mathrm{Cth}}$ of $\mathrm{Y} 103 \mathrm{~F}$ is unique among the site-directed variants investigated. The catalytic efficiency of Y103F-catalyzed L-Cys hydrolysis is altered only 2 -fold from the wild type, but the $K_{\mathrm{i}}^{\mathrm{L}-\mathrm{Cys}}$ of $\mathrm{Y} 103 \mathrm{~F}$ is increased approximately 9-fold while the $K_{\mathrm{m}}{ }^{\mathrm{L}-\mathrm{Cys}}$ is decreased 3-fold (Table 3.6). The kinetic parameters for the Y103F-catalyzed hydrolysis of both L-OAS and L-Hcys are within 3- 
Table 3.6. Kinetic parameters of L-Cth, L-Cys, L-OAS and L-Hcys hydrolysis by yCGL and site-directed variants of residues Y49, Y103, and S334.

\begin{tabular}{|c|c|c|c|c|}
\hline Enzyme & $k_{\text {cat }}\left(\mathrm{s}^{-1}\right)$ & $K_{\mathrm{m}}(\mathrm{mM})$ & $K_{i}^{\text {L-Cys }}(\mathbf{m M})$ & $k_{\text {cat }} / K_{\mathrm{m}}\left(\mathrm{M}^{-1} \mathrm{~s}^{-1}\right)$ \\
\hline \multicolumn{5}{|c|}{ L-Cth $\rightarrow$ L-Cys $+\alpha$-ketobutyrate $+\mathrm{NH}_{3}$} \\
\hline yCGL & $1.51 \pm 0.03$ & $0.71 \pm 0.04$ & & $(2.1 \pm 0.1) \times 10^{3}$ \\
\hline Y49F & $0.008 \pm 0.001$ & $3.0 \pm 0.8$ & & $2.7 \pm 0.4$ \\
\hline Y103F & $0.0036 \pm 0.0001$ & $0.08 \pm 0.02$ & & $46 \pm 8$ \\
\hline S334A & $0.0024 \pm 0.0001$ & $0.23 \pm 0.05$ & & $11 \pm 2$ \\
\hline \multicolumn{5}{|c|}{$\mathrm{L}-\mathrm{Cys} \rightarrow$ pyruvate $+\mathrm{H}_{2} \mathrm{~S}+\mathrm{NH}_{3}$} \\
\hline yCGL & $0.15 \pm 0.01$ & $0.09 \pm 0.01$ & $2 \pm 0.3$ & $(1.7 \pm 0.2) \times 10^{3}$ \\
\hline Y49F & $0.0054 \pm 0.0004$ & $0.09 \pm 0.02$ & $10 \pm 3$ & $(6 \pm 1) \times 10^{1}$ \\
\hline Y103F & $0.24 \pm 0.02$ & $0.29 \pm 0.05$ & $18 \pm 7$ & $(8.4 \pm 0.9) \times 10^{2}$ \\
\hline \multicolumn{5}{|c|}{ L-OAS $\rightarrow$ acetate + pyruvate $+\mathrm{NH}_{3}$} \\
\hline yCGL & $0.0097 \pm 0.0002$ & $2.5 \pm 0.3$ & & $3.8 \pm 0.4$ \\
\hline Y49F & $0.0038 \pm 0.0002$ & $2.3 \pm 0.5$ & & $1.6 \pm 0.3$ \\
\hline Y103F & $0.0046 \pm 0.0001$ & $0.7 \pm 0.1$ & & $6 \pm 1$ \\
\hline \multicolumn{5}{|c|}{ L-Hcys $\rightarrow \alpha$-ketobutyrate $+\mathrm{H}_{2} \mathrm{~S}+\mathrm{NH}_{3}$} \\
\hline yCGL & n.s. ${ }^{\mathrm{a}}$ & n.s. & & $0.027 \pm 0.005$ \\
\hline Y49F & n.s. & n.s. & & $0.013 \pm 0.003$ \\
\hline Y103F & n.s. & n.s. & & $0.042 \pm 0.004$ \\
\hline
\end{tabular}

an.s. indicates that the $K_{\mathrm{m}}$ exceeded the solubility limit of the substrate, such that $k_{\mathrm{cat}} / K_{\mathrm{m}}$ was obtained by linear regression. 

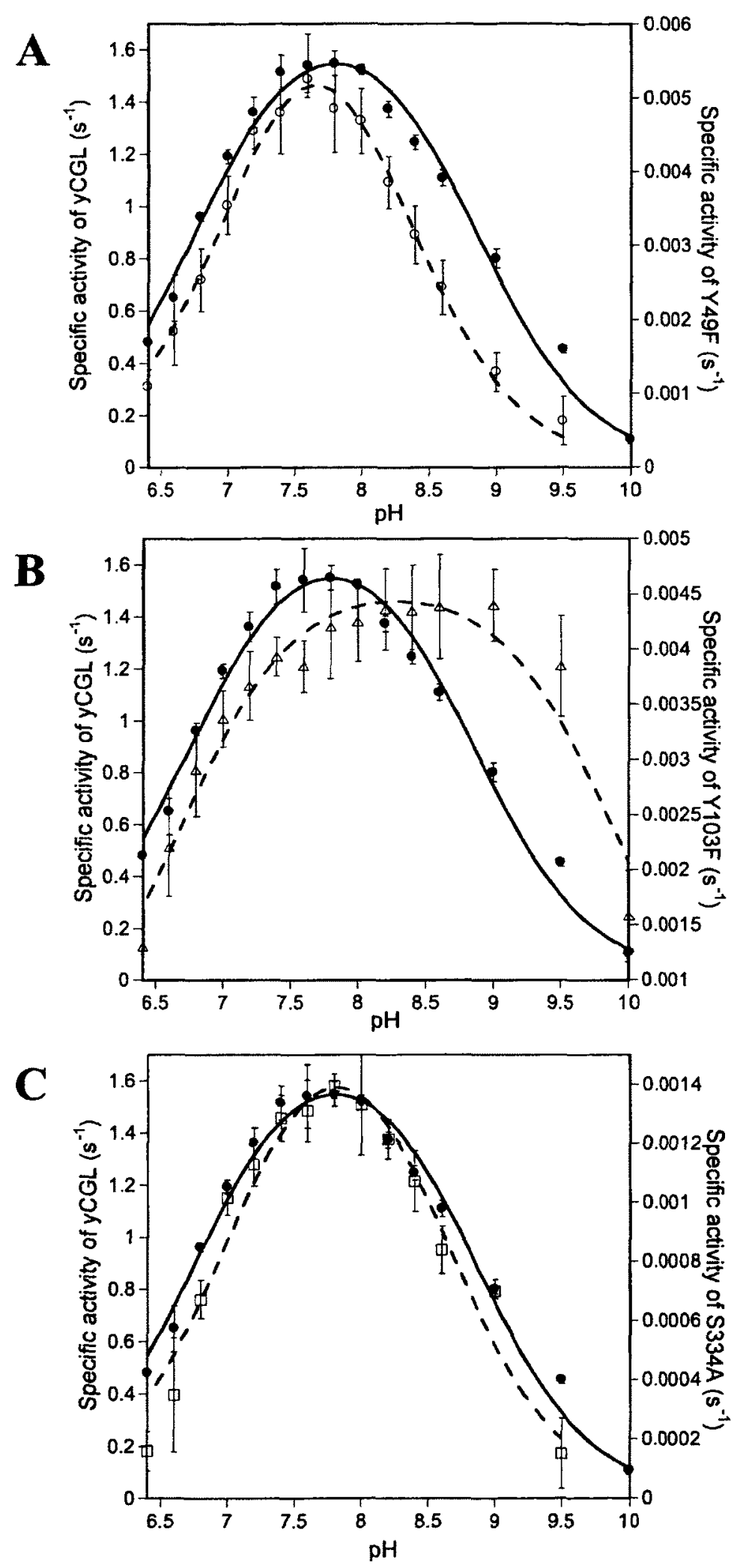

Figure 3.11. Comparison of the $\mathrm{pH}$ dependence of the specific activity of L-Cth hydrolysis for wild-type yCGL (solid circle) and the (A) Y49F (empty circle), (B) Y103F (triangle) and (C) S334A (square) variants. 

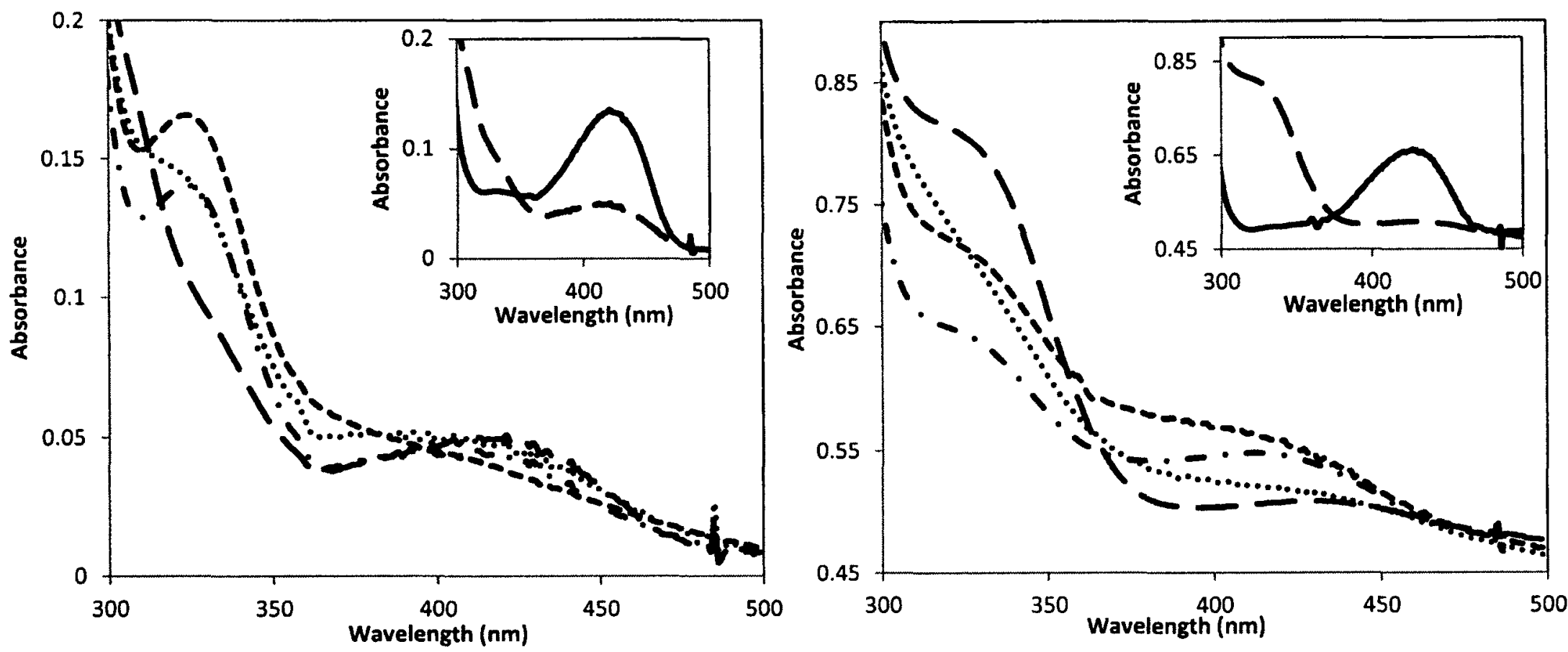

Figure 3.12. The effect of 120-min incubation with (A) $100 \mathrm{mM} \mathrm{L}$-alanine and (B) $3 \mathrm{mM} \mathrm{L}$-Cth on the absorbance spectra of the PLP cofactor of wild-type yCGL (long dashed line) and the Y49F (short dashed line), Y103F (dotted line) and S334A (mixed dotted/dashed line) variants. Insets: spectra of wild-type yCGL before (solid line) and after (long dashed line) 120-min incubation with substrate. 
fold of the wild type (Table 3.6). Interestingly, the optimum of the specific activity versus $\mathrm{pH}$ profile of $\mathrm{Y} 103 \mathrm{~F}$ is shifted to $8.4-9$ (Figure 3.11B), an increase of $\sim 1.1 \mathrm{pH}$ units from wild-type yCGL (Table 3.3), but the $\mathrm{p} K_{a}$ values of the acidic and basic limbs of the bell-shaped $k_{\text {cat }} / K_{\mathrm{m}}$ versus $\mathrm{pH}$ profile of the $\mathrm{Y} 103 \mathrm{~F}$ variant are within experimental error of the wild-type enzyme (Table 3.4; Figure 3.13). The absorbance spectrum of the Y103F variant displays a shoulder at $330 \mathrm{~nm}$ following incubation for $120 \mathrm{~min}$ with $100 \mathrm{mM} \mathrm{L}$ alanine (Figure 3.12A), but the $330 \mathrm{~nm}$ absorbance peak of the wild-type enzyme, following reaction with $\mathrm{L}-\mathrm{Cth}$, is not observed for $\mathrm{Y} 103 \mathrm{~F}$, a feature that distinguishes this enzyme from the wild-type yCGL, as well as the other variants investigated (Figure 3.12B).

The catalytic efficiency of L-Cth hydrolysis by the S334A variant is decreased 190-fold from that of wild-type yCGL, primarily due to a reduction of 630 -fold in $k_{\text {cat }}$, as the $K_{\mathrm{m}}^{\mathrm{L}-\mathrm{Cth}}$ of this variant is also decreased, but by only 3 -fold (Table 3.6). The hydrolysis of L-Hcys, L-Cys, and L-OAS by S334A is undetectable. The specific activity of L-Cth hydrolysis versus $\mathrm{pH}$ profile of the $\mathrm{S} 334 \mathrm{~A}$ variant is similar to that of the wild-type enzyme (Table 3.3; Figure 3.11C). Reduced formation of the 330-nm band after reaction with L-Cth is observed in the absorbance spectrum of S334A, compared to yCGL, and incubation of S334A with $100 \mathrm{mM} \mathrm{L}$-alanine for 120 min results in the appearance of a 330-nm peak absent from the spectrum of the wild-type enzyme (Figure 3.12). 


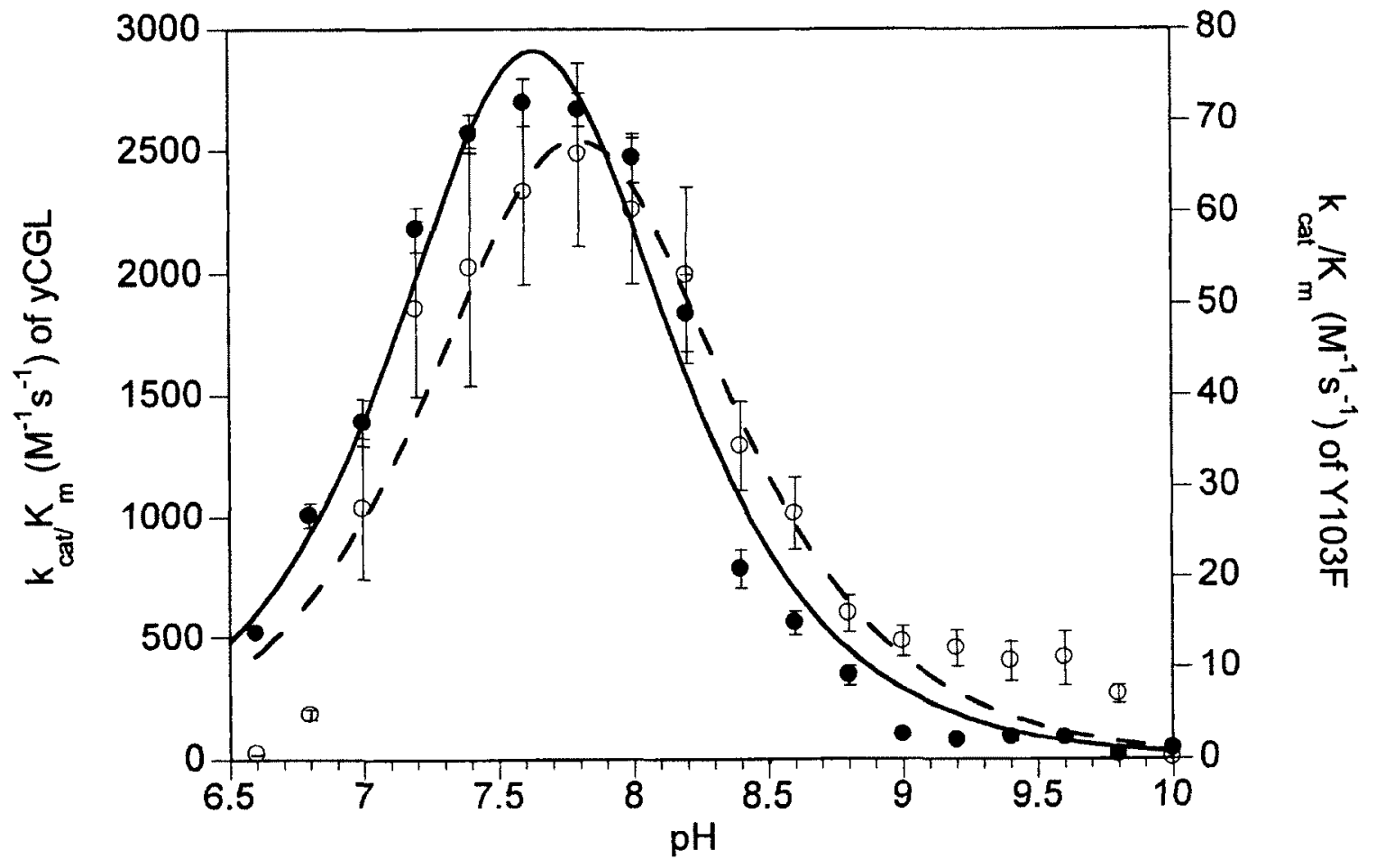

Figure 3.13. Comparison of pH dependence of the catalytic efficiency of L-Cth hydrolysis of wild-type yCGL (solid circle, solid line) and the Y103F (empty circle, dashed line) variant. 


\subsection{The E48A/D/Q variants.}

The catalytic efficiency for the hydrolysis of L-Cth and the $\beta$-elimination of both L-Cys and L-OAS by the yCGL E48A/D/Q variants are within 3-fold of wild-type yCGL (Table 3.7). In contrast, all substitution variants of E48 lack detectable L-Hcys $\gamma$-elimination activity. The $k_{\text {cal }} / K_{\mathrm{m}}^{\mathrm{L}-\mathrm{Cth}}$ versus $\mathrm{pH}$ profiles of the E48A and E48Q substitution variants have optima shifted to $7.8-8.2$ and $7.6-8.2$, respectively (Figure 3.14 ), which represent increases of approximately $0.5 \mathrm{pH}$ units compared to the wild-type enzyme, and basic limb $\mathrm{p} K_{\mathrm{a}}$ values increased by $\sim 0.6 \mathrm{pH}$ units (Table 3.4). In contrast, the $\mathrm{pH}$ optimum and $\mathrm{p} K_{\mathrm{a}}$ values of the $k_{\text {cat }} / K_{\mathrm{m}}{ }^{\mathrm{L}-\text {-Cth }}$ versus pH profile of the E48D variant (Figure 3.14 and Table 3.4), which maintains the carboxylate functionality of the side chain at this position but decreases its length by $\sim 1 \AA$, is within experimental error of the wild type. The specific activity versus $\mathrm{pH}$ profiles of the E48A/D/Q (Table 3.3) variants follow the same trends as the respective $k_{\text {cat }} / K_{\mathrm{m}}{ }^{\text {L-Cth }}$ versus $\mathrm{pH}$ profiles. The absorbance spectra of the PLP cofactor of the E48A/D/Q variants display similar changes to the wild type after incubation for $120 \mathrm{~min}$ with either $100 \mathrm{mM}$ L-alanine or $3 \mathrm{mM} \mathrm{L}$-Cth (Figure 3.15).

\subsection{The $E 333 A / D / Q$ variants.}

The alanine, aspartate, and glutamine substitutions of E333 result in an increase in $K_{\mathrm{m}}^{\text {L-Cth }}$ of 16-18-fold, while the $k_{\text {cat }}$ for hydrolysis of L-Cth by these variants is within 3-fold of the wild type (Table 3.8). The catalytic efficiency of L-Cys hydrolysis by the E333A/D/Q variants is 
Table 3.7. Kinetic parameters of L-Cth, L-Cys, L-OAS and L-Hcys hydrolysis by yCGL and sitedirected variants of residue $\mathrm{E} 48$.

\begin{tabular}{|c|c|c|c|c|}
\hline Enzyme & $k_{\text {cat }}\left(s^{-1}\right)$ & $K_{\mathrm{m}}(\mathrm{mM})$ & $K_{i}^{\text {L-Cys }}(\mathbf{m M})$ & $k_{\mathrm{cat}} / K_{\mathrm{m}}\left(\mathrm{M}^{-1} \mathrm{~s}^{-1}\right)$ \\
\hline \multicolumn{5}{|c|}{ L-Cth $\rightarrow$ L-Cys $+\alpha$-ketobutyrate $+\mathrm{NH}_{3}$} \\
\hline yCGL & $1.51 \pm 0.03$ & $0.71 \pm 0.04$ & & $(2.1 \pm 0.1) \times 10^{3}$ \\
\hline E48A & $0.54 \pm 0.01$ & $0.76 \pm 0.04$ & & $(7.1 \pm 0.3) \times 10^{2}$ \\
\hline E48D & $1.4 \pm 0.02$ & $0.87 \pm 0.04$ & & $(1.59 \pm 0.05) \times 10^{3}$ \\
\hline E48Q & $1.29 \pm 0.04$ & $0.9 \pm 0.1$ & & $(1.4 \pm 0.1) \times 10^{3}$ \\
\hline \multicolumn{5}{|c|}{ L-Cys $\rightarrow$ pyruvate $+\mathrm{H}_{2} \mathrm{~S}+\mathrm{NH}_{3}$} \\
\hline yCGL & $0.15 \pm 0.01$ & $0.09 \pm 0.01$ & $2 \pm 0.3$ & $(1.7 \pm 0.2) \times 10^{3}$ \\
\hline E48A & $0.09 \pm 0.01$ & $0.11 \pm 0.02$ & $3.1 \pm 0.6$ & $(8 \pm 1) \times 10^{2}$ \\
\hline E48D & $0.32 \pm 0.04$ & $0.4 \pm 0.1$ & $2.2 \pm 0.6$ & $(7.2 \pm 0.8) \times 10^{2}$ \\
\hline E48Q & $0.18 \pm 0.01$ & $0.18 \pm 0.02$ & $5.7 \pm 0.7$ & $(1.02 \pm 0.06) \times 10^{3}$ \\
\hline \multicolumn{5}{|c|}{ L-OAS $\rightarrow$ acetate + pyruvate $+\mathrm{NH}_{3}$} \\
\hline yCGL & $0.0097 \pm 0.0002$ & $2.5 \pm 0.3$ & & $3.8 \pm 0.4$ \\
\hline $\mathbf{E 4 8 A}$ & $0.0142 \pm 0.0004$ & $1.5 \pm 0.2$ & & $10 \pm 1$ \\
\hline E48D & $0.0053 \pm 0.0001$ & $1.4 \pm 0.1$ & & $3.8 \pm 0.3$ \\
\hline E48Q & $0.0124 \pm 0.0002$ & $4.4 \pm 0.3$ & & $2.8 \pm 0.2$ \\
\hline \multicolumn{5}{|c|}{ L-Hcys $\rightarrow \alpha$-ketobutyrate $+\mathrm{H}_{2} \mathrm{~S}+\mathrm{NH}_{3}$} \\
\hline yCGL & n.s. ${ }^{\text {a }}$ & n.s. & & $0.027 \pm 0.005$ \\
\hline
\end{tabular}

${ }^{2}$ n.s. indicates that the $K_{\mathrm{m}}$ exceeded the solubility limit of the substrate, such that $k_{\text {cat }} / K_{\mathrm{m}}$ was obtained by linear regression. 


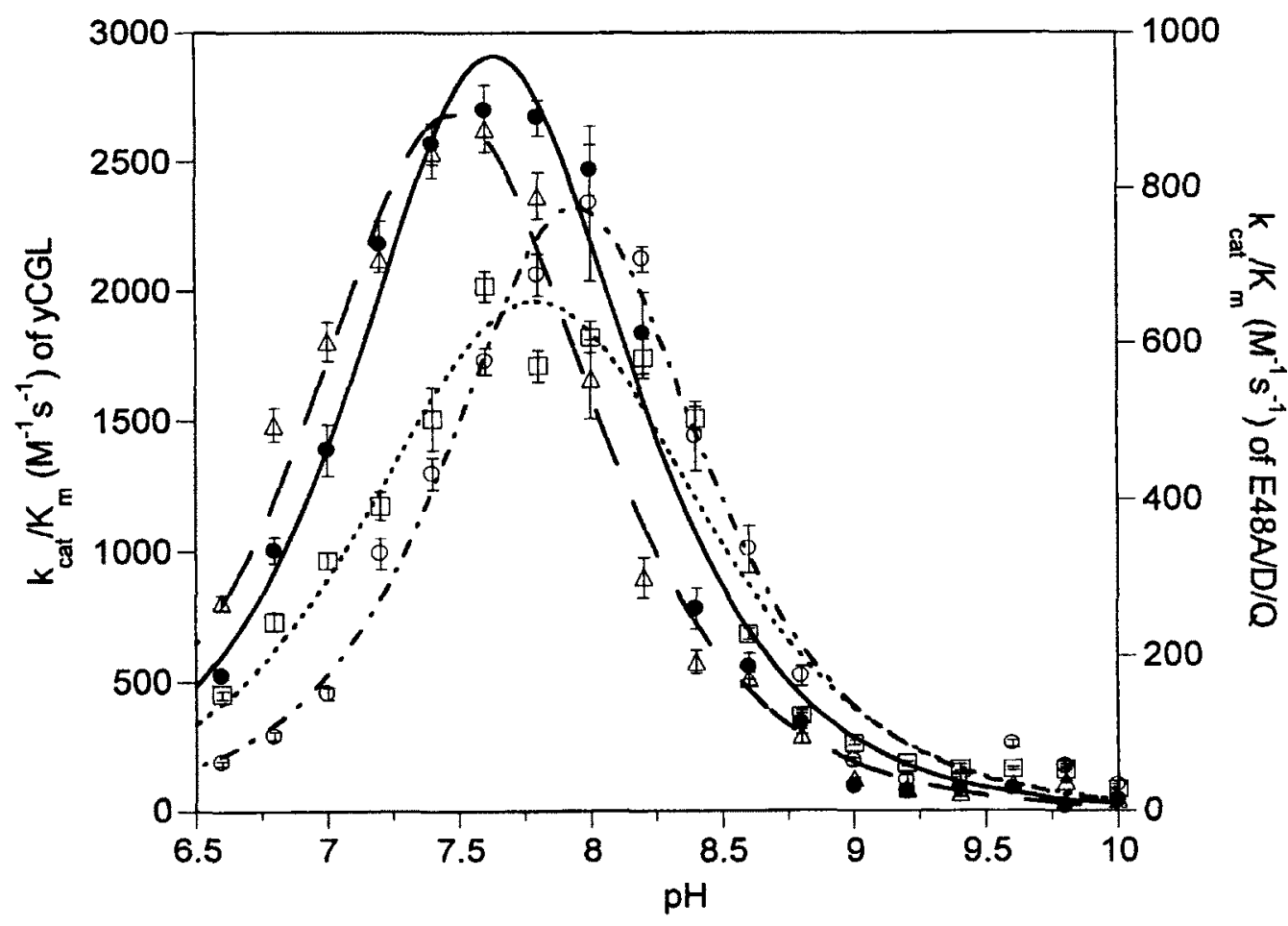

Figure 3.14. The $\mathrm{pH}$ dependence of the catalytic efficiency of L-Cth hydrolysis of wild-type yCGL (solid circle, solid line) and the E48A (empty circle, mixed dotted/dashed line), E48D (triangle, long dashed line), and E48Q (square, dotted line) variants. 

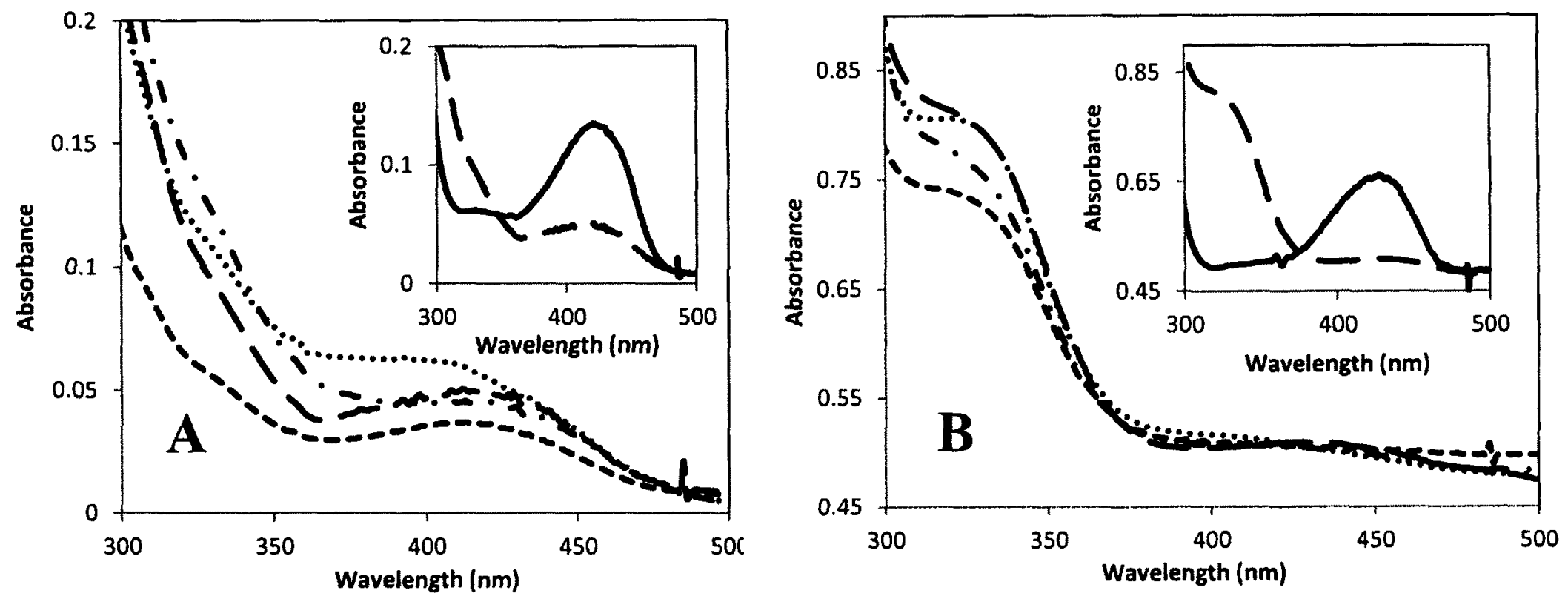

Figure 3.15. The effect of 120-min incubation with (A) $100 \mathrm{mM} \mathrm{L}$-alanine and (B) $3 \mathrm{mM} \mathrm{L}-\mathrm{Cth}$ on the absorbance spectra of the PLP cofactor of wild-type yCGL (long dashed line) and the E48A (short dashed line), E48D (dotted line), and E48Q (mixed dotted/dashed line) variants. Insets: spectra of wild-type yCGL before (solid line) and after (long dashed line) 120-min incubation with substrate. 
Table 3.8. Kinetic parameters of L-Cth, L-Cys, L-OAS and L-Hcys hydrolysis by yCGL and site-directed variants of residue E333.

\begin{tabular}{|c|c|c|c|c|}
\hline Enzyme & $k_{\text {cat }}\left(\mathrm{s}^{-1}\right)$ & $K_{\mathbf{m}}(\mathbf{m M})$ & $K_{i}^{\text {L-Cys }}(\mathbf{m M})$ & $k_{\mathrm{cat}} / K_{\mathrm{m}}\left(\mathrm{M}^{-1} \mathrm{~s}^{-1}\right)$ \\
\hline \multicolumn{5}{|c|}{ L-Cth $\rightarrow \mathrm{L}-\mathrm{Cys}+\alpha$-ketobutyrate $+\mathrm{NH}_{3}$} \\
\hline yCGL & $1.51 \pm 0.03$ & $0.71 \pm 0.04$ & & $(2.1 \pm 0.1) \times 10^{3}$ \\
\hline E333A & $0.78 \pm 0.05$ & $12 \pm 1$ & & $65 \pm 1$ \\
\hline E333D & $0.98 \pm 0.04$ & $12 \pm 1$ & & $82 \pm 1$ \\
\hline E333Q & $0.48 \pm 0.09$ & $13 \pm 3$ & & $36 \pm 2$ \\
\hline \multicolumn{5}{|c|}{ L-Cys $\rightarrow$ pyruvate $+\mathrm{H}_{2} \mathrm{~S}+\mathrm{NH}_{3}$} \\
\hline yCGL & $0.15 \pm 0.01$ & $0.09 \pm 0.01$ & $2 \pm 0.3$ & $(1.7 \pm 0.2) \times 10^{3}$ \\
\hline E333A & $1.3 \pm 0.5$ & $1.2 \pm 0.6$ & $0.8 \pm 0.4$ & $(8.1 \pm 0.7) \times 10^{2}$ \\
\hline E333D & $0.24 \pm 0.04$ & $1.8 \pm 0.4$ & $1.1 \pm 0.3$ & $(1.3 \pm 0.1) \times 10^{2}$ \\
\hline E333Q & $0.5 \pm 0.1$ & $0.7 \pm 0.2$ & $3 \pm 1$ & $(4.9 \pm 0.3) \times 10^{2}$ \\
\hline \multicolumn{5}{|c|}{ L-OAS $\rightarrow$ acetate + pyruvate $+\mathrm{NH}_{3}$} \\
\hline yCGL & $0.0097 \pm 0.0002$ & $2.5 \pm 0.3$ & & $3.8 \pm 0.4$ \\
\hline E333A & $0.0058 \pm 0.0002$ & $2.2 \pm 0.3$ & & $2.6 \pm 0.3$ \\
\hline E333D & $0.0045 \pm 0.0002$ & $0.9 \pm 0.2$ & & $5 \pm 1$ \\
\hline E333Q & $0.0102 \pm 0.0002$ & $5.3 \pm 0.4$ & & $1.9 \pm 0.1$ \\
\hline \multicolumn{5}{|c|}{ L-Hcys $\rightarrow \alpha$-ketobutyrate $+\mathrm{H}_{2} \mathrm{~S}+\mathrm{NH}_{3}$} \\
\hline yCGL & n.s. ${ }^{a}$ & n.s. & & $0.027 \pm 0.005$ \\
\hline E333D & n.s. & n.s. & & $0.03 \pm 0.01$ \\
\hline E333Q & n.s. & n.s. & & $0.03 \pm 0.01$ \\
\hline
\end{tabular}

${ }^{a}$.s. indicates that the $K_{\mathrm{m}}$ exceeded the solubility limit of the substrate, such that $k_{\mathrm{cat}} / K_{\mathrm{m}}$ was obtained by linear regression. 
within one order of magnitude of the wild type, an effect dominated by the $\sim 8-20$-fold increases in $K_{\mathrm{m}}^{\text {L-Cys }}$ observed for these enzymes (Table 3.8). Substitution of E333 with alanine, aspartate or glutamine alters the catalytic efficiency of L-OAS $\beta$-elimination by less than 2.5-fold, compared to the wild-type enzyme (Table 3.8). The hydrolysis of LHcys is undetectable for yCGL-E333A, while the $k_{\text {cat }} / K_{\mathrm{m}}{ }^{\text {L-Hcys }}$ of the E333D/Q variants are within experimental error of wild-type yCGL (Table 3.8).

Substitution of residue E333 with alanine or glutamine results in a shift in the $\mathrm{pH}$ optimum of $k_{\text {cat }} / K_{\mathrm{m}}{ }^{\mathrm{L}-\mathrm{Cth}}$ to $8.2-8.6$, which represents an increase of approximately $1 \mathrm{pH}$ unit compared to the wild-type enzyme, while the $\mathrm{pH}$ optimum of E333D is similar to the wild type (Figure 3.16). The $\mathrm{p} K_{\mathrm{a}}$ values of the acidic limb of the $k_{\text {cat }} / K_{\mathrm{m}}{ }^{\mathrm{L}-\mathrm{Cth}} \mathrm{pH}$ profiles of the E333A and E333Q variants are increased by $0.6-1 \mathrm{pH}$ unit, while both $\mathrm{p} K_{\mathrm{a}}$ values of E333D are within experimental error of wild-type yCGL (Tables 3.4).

Although similar spectral changes to the wild type were observed for the $\mathrm{E} 333 \mathrm{~A} / \mathrm{D} / \mathrm{Q}$ variants upon reaction with $3 \mathrm{mM} \mathrm{L}-\mathrm{Cth}$, incubation for $120 \mathrm{~min}$ with 100 $\mathrm{mM} \mathrm{L-alanine} \mathrm{results} \mathrm{in} \mathrm{the} \mathrm{appearance} \mathrm{of} \mathrm{a} \mathrm{330-nm} \mathrm{peak} \mathrm{in} \mathrm{the} \mathrm{spectrum} \mathrm{of} \mathrm{the} \mathrm{cofactor}$ of the $\mathrm{E} 333 \mathrm{~A} / \mathrm{D} / \mathrm{Q}$ variants, a feature that is absent in the spectrum of the wild-type enzyme (Figure 3.17).

\subsection{The E48/E333 alanine, aspartate, and glutamine double replacement variants.}

The alanine, aspartate and glutamine double replacement variants of residues E48/E333, constructed to investigate the possibility of additive effects of two residues 

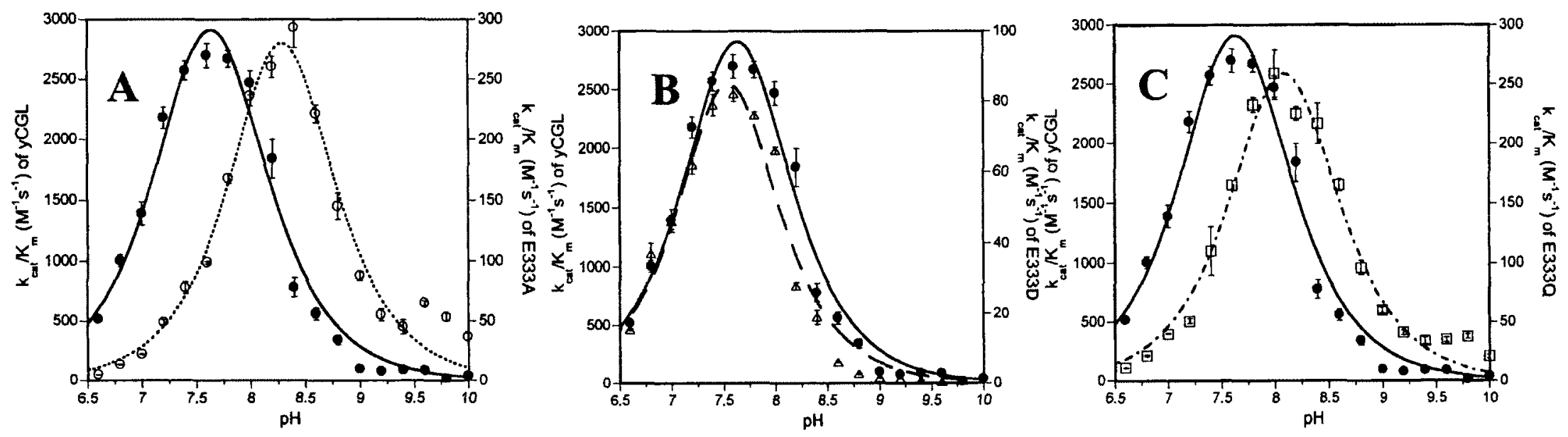

Figure 3.16. The $\mathrm{pH}$ dependence of the catalytic efficiency of L-Cth hydrolysis for wild-type yCGL (solid circle, solid line) and the (A) E333A (empty circle, dotted line), (B) E333D (triangle, long dashed line), and (C) E333Q (square, mixed dotted/dashed line) variants. 

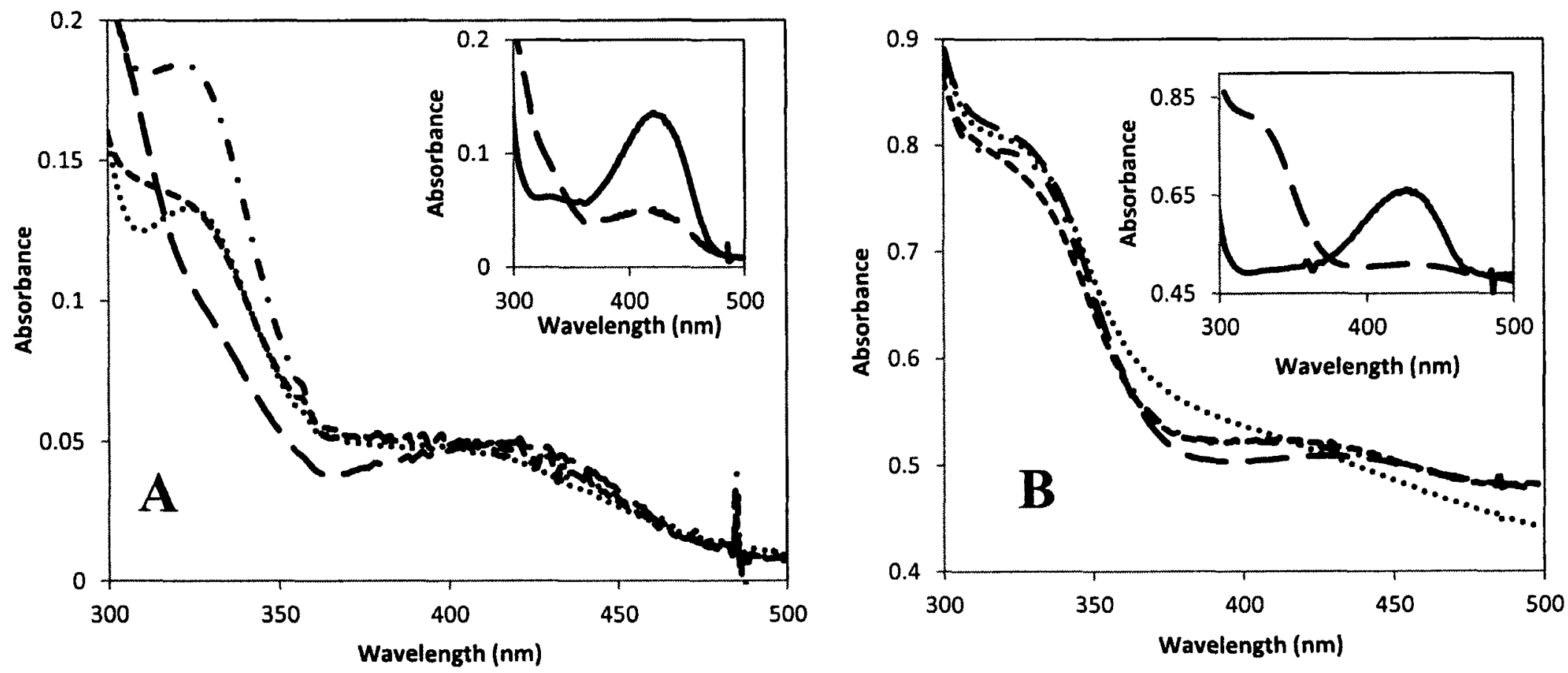

Figure 3.17. The effect of 120-min incubation with (A) $100 \mathrm{mM} \mathrm{L}$-alanine and (B) $3 \mathrm{mM} \mathrm{L}$-Cth on the absorbance spectra of the PLP cofactor of wild-type yCGL (long dashed line) and the E333A (short dashed line), E333D (dotted line), and E333Q (mixed dotted/dashed line) variants. Insets: spectra of wild-type yCGL before (solid line) and after (long dashed line) 120-min incubation with substrate. 
proposed to modulate the specificity of yCGL, are not saturated within the solubility limit of the L-Cth substrate, and the catalytic efficiency for hydrolysis of the L-Cth substrate by these variants is reduced by 560,240 , and 125 -fold, respectively, compared to yCGL (Table 3.9). The value of $K_{\mathrm{m}}^{\mathrm{L}-\mathrm{Cys}}$ is increased 4 - 5-fold for all double replacement variants of E48/E333 and the $k_{\text {cat }}$ of the E48A/E333A variant for L-Cys hydrolysis is 13 fold lower than the wild type (Table 3.9). With the exception of the $K_{\mathrm{m}}^{\text {L-OAS }}$ of $\mathrm{E} 48 \mathrm{D} / \mathrm{E} 333 \mathrm{D}$, which is decreased 3.5 -fold, the kinetic parameters for hydrolysis of LOAS by the E48/E333 double replacement variants are within 2.5-fold of the wild-type enzyme (Table 3.9). The catalytic efficiency of L-Hcys hydrolysis for the alanine, aspartate and glutamine E48/E333 variants is within 3-fold of wild-type yCGL (Table 3.9).

Substitution of both E48 and E333 residues with alanine or glutamine results in shifts in the $\mathrm{pH}$ optimum of $k_{\text {cat }} / K_{\mathrm{m}}^{\text {L-Cth }}$ to $9.2-10$ and $8.9-9.2$, respectively (Figure 3.18). This represents increases of $1.5-2 \mathrm{pH}$ units compared to wild-type yCGL, and $\sim 1$ $\mathrm{pH}$ unit compared to the corresponding single substitution variants of E48 (Figure 3.14) and E333 (Figure 3.16). In contrast, the $\mathrm{pH}$ optimum of $k_{\text {cat }} / K_{\mathrm{m}}{ }^{\mathrm{L} C \mathrm{Cth}}$ for E48D/E333D is increased by only $0.8 \mathrm{pH}$ units (Figure 3.18 ). The $\mathrm{p} K_{\mathrm{a}}$ values of both the basic and acidic limbs of $k_{\text {cat }} / K_{\mathrm{m}}{ }^{\text {L-Cth }}$ versus $\mathrm{pH}$ for E48A/E333A, E48D/E333D and E48Q/E333Q are altered $0.5-1.2 \mathrm{pH}$ units, respectively, compared to the wild-type enzyme (Table 3.4).

The spectral characteristics of the cofactor of the E48/E333 double replacement variants (Figure 3.19) are similar to those of the E333A/D/Q single substitution variants (Figure 3.17). Following 120-min reaction with L-Cth a 330-nm peak, similar to that observed for wild-type yCGL and the E48A/D/Q and E333A/D/Q single substitution 
Table 3.9. Kinetic parameters of L-Cth, L-Cys, L-OAS and L-Hcys hydrolysis by yCGL and site-directed double replacement variants of residues E48/E333.

\begin{tabular}{|c|c|c|c|c|}
\hline Enzyme & $k_{\mathrm{cat}}\left(\mathrm{s}^{-1}\right)$ & $K_{\mathrm{m}}(\mathrm{mM})$ & $K_{i}^{\text {L-Cys }}(\mathbf{m M})$ & $k_{\mathrm{cat}} / K_{\mathrm{m}}\left(\mathrm{M}^{-1} \mathrm{~s}^{-1}\right)$ \\
\hline \multicolumn{5}{|c|}{$\mathrm{L}-\mathrm{Cth} \rightarrow \mathrm{L}-\mathrm{Cys}+\alpha$-ketobutyrate $+\mathrm{NH}_{3}$} \\
\hline yCGL & $1.51 \pm 0.03$ & $0.71 \pm 0.04$ & & $(2.1 \pm 0.1) \times 10^{3}$ \\
\hline E48A/E333A & n.s. ${ }^{a}$ & n.s. & & $3.7 \pm 0.1$ \\
\hline E48D/E333D & n.s. & n.s. & & $8.8 \pm 0.1$ \\
\hline E48Q/E333Q & n.s. & n.s. & & $16.9 \pm 0.1$ \\
\hline \multicolumn{5}{|c|}{$\mathrm{L}-\mathrm{Cys} \rightarrow$ pyruvate $+\mathrm{H}_{2} \mathrm{~S}+\mathrm{NH}_{3}$} \\
\hline yCGL & $0.15 \pm 0.01$ & $0.09 \pm 0.01$ & $2 \pm 0.3$ & $(1.7 \pm 0.2) \times 10^{3}$ \\
\hline E48A/E333A & $0.011 \pm 0.02$ & $0.5 \pm 0.1$ & $0.4 \pm 0.1$ & $(2.1 \pm 0.1) \times 10^{2}$ \\
\hline E48D/E333D & $0.029 \pm 0.004$ & $0.5 \pm 0.1$ & $0.9 \pm 0.2$ & $(6 \pm 1) \times 10^{1}$ \\
\hline E48Q/E333Q & $0.16 \pm 0.04$ & $0.4 \pm 0.1$ & $0.7 \pm 0.2$ & $(3.1 \pm 0.2) \times 10^{2}$ \\
\hline \multicolumn{5}{|c|}{ L-OAS $\rightarrow$ acetate + pyruvate $+\mathrm{NH}_{3}$} \\
\hline yCGL & $0.0097 \pm 0.0002$ & $2.5 \pm 0.3$ & & $3.8 \pm 0.4$ \\
\hline E48A/E333A & $0.0047 \pm 0.0002$ & $2.9 \pm 0.6$ & & $1.6 \pm 0.3$ \\
\hline E48D/E333D & $0.0035 \pm 0.0002$ & $0.7 \pm 0.2$ & & $5 \pm 1$ \\
\hline E48Q/E333Q & $0.0092 \pm 0.0004$ & $1.5 \pm 0.3$ & & $6 \pm 1$ \\
\hline \multicolumn{5}{|c|}{ L-Hcys $\rightarrow \alpha$-ketobutyrate $+\mathrm{H}_{2} \mathrm{~S}+\mathrm{NH}_{3}$} \\
\hline yCGL & n.s. & n.s. & & $0.027 \pm 0.005$ \\
\hline E48A/E333A & n.s. & n.s. & & $0.014 \pm 0.003$ \\
\hline E48D/E333D & n.s. & n.s. & & $0.09 \pm 0.04$ \\
\hline E48Q/E333Q & n.s. & n.s. & & $0.04 \pm 0.01$ \\
\hline
\end{tabular}

n.s. indicates that the $K_{\mathrm{m}}$ exceeded the solubility limit of the substrate, such that $k_{\mathrm{cal}} / K_{\mathrm{m}}$ was obtained by linear regression. 


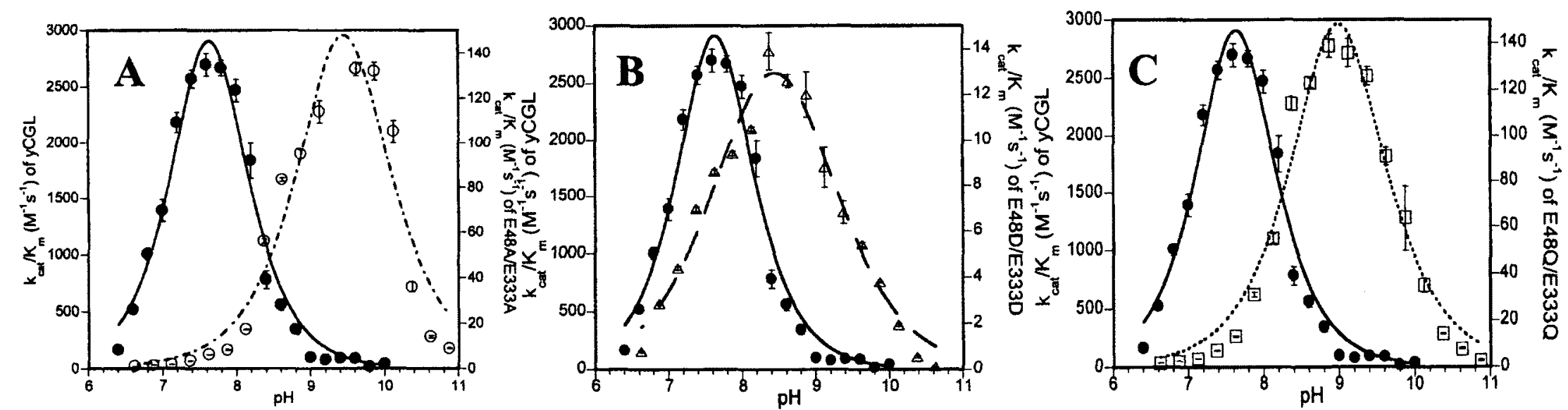

Figure 3.18. Comparison of the $\mathrm{pH}$ dependence of the catalytic efficiency of L-Cth hydrolysis for wild-type yCGL (solid circle) and the (A) E48A/E333A (empty circle), (B) E48D/E333D (triangle), and (C) E48Q/E333Q (square) variants. 

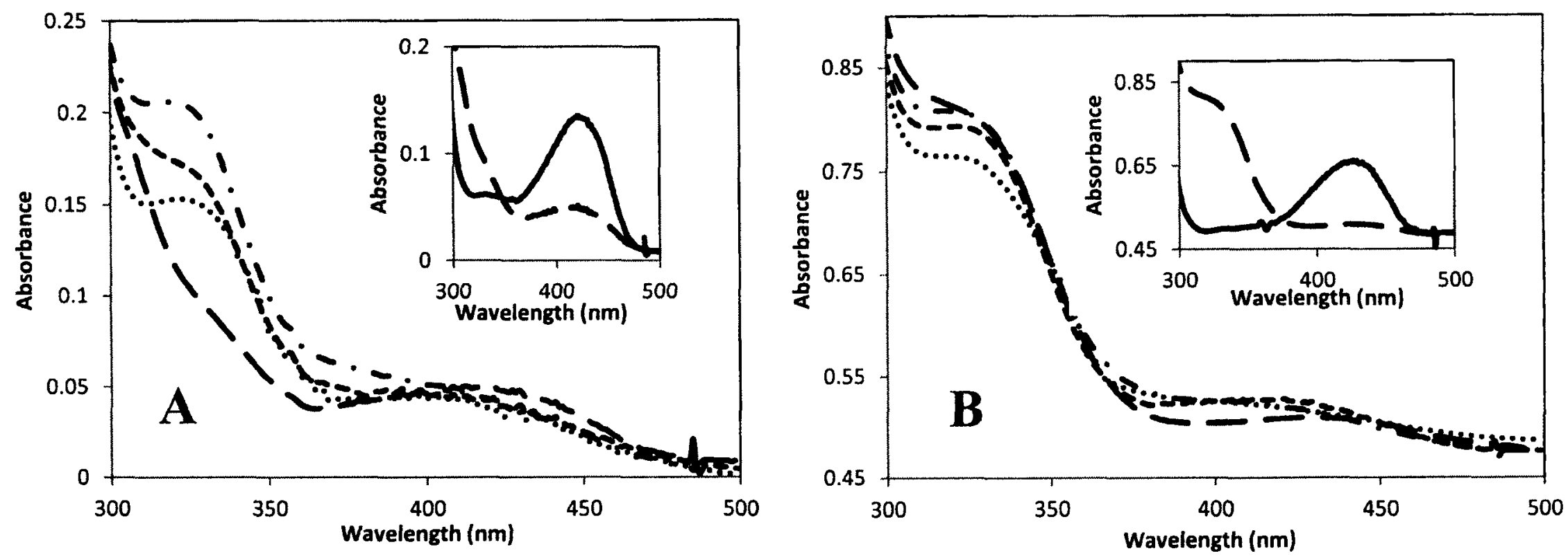

Figure 3.19. The effect of 120-min incubation with (A) $100 \mathrm{mM} \mathrm{L-alanine}$ and (B) $3 \mathrm{mM} \mathrm{L-Cth}$ on the absorbance spectra of the PLP cofactor of wild-type yCGL (long dashed line) and the E48A/E333A (short dashed line), E48D/E333D (dotted line) and E48Q/E333Q (mixed dotted/dashed line) variants. Insets: spectra of wild-type yCGL before (solid line) and after (long dashed line) 120-min incubation with substrate. 
variants, is present in the spectra of the alanine, aspartate and glutamine substitutions of E48/E333 (Figure 3.19B). However, 120-min incubation with $100 \mathrm{mM}$ L-alanine results in formation of a 330-nm peak in the spectra of E48A/E333A, E48D/E333D, and $\mathrm{E} 48 \mathrm{Q} / \mathrm{E} 333 \mathrm{Q}$ (Figure 3.19A), which is similar to the behaviour of the E333A/D/Q single substitution variants, but not observed for the wild-type enzyme or the $E 48 \mathrm{~A} / \mathrm{D} / \mathrm{Q}$ variants. 


\section{DISCUSSION}

The catalytic versatility of the PLP cofactor is demonstrated by the wide range of amino acid transformations catalyzed by PLP-dependent enzymes, such as transamination, decarboxylation, and $\beta / \gamma$ side chain rearrangements. However, this means that the specificity required for these reactions must be provided by the enzyme, primarily the active-site residues responsible for substrate-binding and catalysis. The $\gamma$ subfamily enzymes, of the PLP-dependent fold type I structural class, of the transsulfuration pathways provide a model system for the investigation of the determinants of substrate and reaction specificity, and the roles of active-site residues of eCGS and eCBL have been demonstrated (Aitken et al., 2003; Farsi et al., 2009; Lodha et al., 2010; Lodha and Aitken, 2011; Jaworski et al., 2012). The focus of this study is the investigation of the effect of 21 site-directed replacements of 10 residues in the active site of yCGL (Table 4.1), and comparison to the demonstrated functions of corresponding residues of eCGS and eCBL (Table 4.2), with the goal of characterizing the roles of these active-site residues as determinants of specificity.

\subsection{The wild-type $y C G L$ enzyme.}

The catalytic efficiencies of the $\beta$-elimination of L-Cys and the physiological hydrolysis of L-Cth differ by only 1.2-fold (Table 3.2), demonstrating the low reaction specificity of yCGL. This is consistent with the findings of Yamagata and co-workers, who reported that yCGL lacks $\beta / \gamma$-elimination selectivity (Yamagata et al., 1993). In contrast, the catalytic efficiency of hCGL for the $\beta$-elimination of L-Cys is 30 -fold lower 
Table 4.1. Summary of effects of site-directed variants on kinetic parameters, pH dependence, and absorbance spectra of yCGL.

\begin{tabular}{|c|c|c|c|c|c|c|}
\hline Variant(s) & $\begin{array}{c}\text { Kinetic } \\
\text { parameters with } \\
\text { L-Cth }\end{array}$ & $\begin{array}{l}\text { Kinetic } \\
\text { parameters } \\
\text { with L-Cys }\end{array}$ & $\begin{array}{c}\text { Kinetic } \\
\text { parameters } \\
\text { with L-OAS }\end{array}$ & $\begin{array}{l}\text { Kinetic } \\
\text { parameters } \\
\text { with L-Hcys }\end{array}$ & pH dependence & $\begin{array}{l}\text { Spectra with } \\
\text { L-alanine (A) } \\
\text { or L-Cth (C) }\end{array}$ \\
\hline E48A/D/Q & n.c. ${ }^{a}$ & n.c. & n.c. & n.a. ${ }^{b}$ & $\begin{array}{c}0.6 \mathrm{pH} \text { unit } \\
\text { increase in basic } \\
\mathrm{p} K_{\mathrm{a}}(\mathrm{E} 48 \mathrm{~A} / \mathrm{Q})\end{array}$ & n.c. \\
\hline Y49F & $\begin{array}{c}190-\text { fold } k_{\text {cat }} \\
\text { decrease }\end{array}$ & $\begin{array}{c}27 \text {-fold } k_{\text {cat }} \\
\text { decrease }\end{array}$ & n.c. & n.c. & n.c. & $330 \mathrm{~nm}$ peak (A) \\
\hline R51A/K & n.s. ${ }^{c}$ & n.a. & $\begin{array}{c}\text { n.a. (R51A), } \\
\text { n.s. (R51K) }\end{array}$ & n.c. & $0.5 \mathrm{pH}$ unit shifts & $330 \mathrm{~nm}$ peak (A) \\
\hline S52A/R & n.c. & $\begin{array}{l}\text { 18-fold } K_{\mathrm{m}} \\
\text { increase }\end{array}$ & n.c. & n.c. & n.c. & n.d. ${ }^{\mathrm{d}}$ \\
\hline Y103F & $\begin{array}{c}420 \text {-fold } k_{\text {cat }} \\
\text { decrease }\end{array}$ & n.c. & n.c. & n.c. & n.c. & $\begin{array}{l}330 \mathrm{~nm} \text { peak }(\mathrm{A}) ; \text { no } \\
330 \mathrm{~nm} \text { peak }(\mathrm{C})\end{array}$ \\
\hline R108A/K & $\begin{array}{c}8-15 \text {-fold } k_{\text {cat }} \text { and } \\
K_{\mathrm{m}} \text { effects }\end{array}$ & $\begin{array}{l}\leq 13 \text {-fold shifts } \\
\text { in } k_{\text {cat }} \text { and } K_{\mathrm{m}}\end{array}$ & n.a. & $\begin{array}{l}\text { n.c. }(\mathrm{R} 108 \mathrm{~A}) \text {, } \\
\text { n.d. }(\mathrm{R} 108 \mathrm{~K})\end{array}$ & $0.5 \mathrm{pH}$ unit shifts & n.d. \\
\hline $\mathbf{N 2 3 2 A}$ & $\begin{array}{l}21 \text {-fold } K_{\mathrm{m}} \\
\text { increase }\end{array}$ & $\begin{array}{l}11 \text {-fold } K_{\mathrm{m}} \\
\text { increase }\end{array}$ & n.s. & n.a. & $\begin{array}{c}0.8 \mathrm{pH} \text { unit } \\
\text { increase in acidic } \\
\mathrm{p} K_{\mathrm{a}}\end{array}$ & n.d. \\
\hline $\begin{array}{c}\mathrm{E} 333 \mathrm{~A} / \mathrm{D} / \mathrm{Q} \\
\text { and double } \\
\text { mutants }\end{array}$ & $\begin{array}{c}\text { 16-18-fold } K_{\mathrm{m}} \\
\text { increases } \\
\text { (E333A/D/Q); n.s. } \\
\text { for doubles }\end{array}$ & $\begin{array}{l}\text { 5-20-fold } K_{\mathrm{m}} \\
\text { increases }\end{array}$ & n.c. & n.c. & $\begin{array}{c}0.6-2 \mathrm{pH} \text { unit } \\
\text { increase in acidic } \\
\mathrm{p} K_{\mathrm{a}}(\mathrm{A} / \mathrm{Q} \text { variants })\end{array}$ & $330 \mathrm{~nm}$ peak $(\mathrm{A})$ \\
\hline S334A & $\begin{array}{c}630 \text {-fold } k_{\text {cat }} \\
\text { decrease }\end{array}$ & n.a. & n.a. & n.a. & n.c. & $330 \mathrm{~nm}$ peak (A) \\
\hline R369A/K & n.s. & n.a. & n.a. & n.a. & n.d. & n.d. \\
\hline
\end{tabular}

${ }^{2}$ n.c.: only minor changes (<3-fold) from the wild-type observed; ${ }^{b}$ n.a.: no activity detected; ${ }^{n}$ n.s.: saturation not observed with this substrate; ${ }^{d}$ n.d.: not determined. 
Table 4.2. Comparison of roles of active-site residues in yCGL, eCBL, and eCGS.

\begin{tabular}{|c|c|c|c|c|}
\hline $\begin{array}{l}\text { yCGL } \\
\text { residue }\end{array}$ & Role of yCGL residue & $\begin{array}{l}\text { Role of corresponding eCBL } \\
\text { residue }^{\mathrm{a}, \mathrm{b}, \mathrm{c}}\end{array}$ & $\begin{array}{l}\text { Role of corresponding eCGS } \\
\text { residue }^{d}\end{array}$ & $\begin{array}{l}\text { Determinant of reaction } \\
\text { specificity (RS) or } \\
\text { substrate specificity (SS) }\end{array}$ \\
\hline E48 & $\begin{array}{l}\text { Affects charge distribution; not } \\
\text { involved in substrate binding or } \\
\text { catalysis }\end{array}$ & $\begin{array}{l}\text { Not involved in substrate } \\
\text { binding or catalysis }\end{array}$ & $\begin{array}{l}\text { Not involved in substrate } \\
\text { binding or catalysis }\end{array}$ & No. \\
\hline Y49 & $\begin{array}{c}\text { Facilitates catalytic step } \\
\text { common to } \beta \text { - and } \gamma- \\
\text { elimination; may affect } \\
\text { specificity through bond with } \\
\text { PLP phosphate }\end{array}$ & $\begin{array}{l}\text { Not involved in catalysis; } \\
\text { altered pH profile and } K_{\mathrm{m}} \\
\text { increase likely due to loss of } \\
\text { bond with PLP }\end{array}$ & $\begin{array}{l}\text { Not involved in catalysis; } K_{\mathrm{m}} \\
\text { increase likely due to loss of } \\
\text { bond with PLP }\end{array}$ & $\begin{array}{l}\text { Partial determinant of RS } \\
\text { through interaction with } \\
\text { PLP (CGS, CBL, and } \\
\text { CGL), and with catalytic } \\
\text { lysine (CGL). }\end{array}$ \\
\hline $\mathbf{R 5 1}$ & $\begin{array}{l}\text { Binds L-Cth distal carboxylate } \\
\text { and PLP phosphate }\end{array}$ & $\begin{array}{c}\text { Binds L-Cth distal carboxylate } \\
\text { and PLP phosphate }\end{array}$ & $\begin{array}{c}\text { Binds L-OSHS distal } \\
\text { carboxylate and PLP } \\
\text { phosphate }\end{array}$ & $\begin{array}{c}\text { RS, through cofactor } \\
\text { orientation (CGS, CBL } \\
\text { and CGL). }\end{array}$ \\
\hline S52 & $\begin{array}{l}\text { Not involved in substrate } \\
\text { binding or catalysis }\end{array}$ & $\begin{array}{l}\text { Not involved in substrate } \\
\text { binding or catalysis }\end{array}$ & $\begin{array}{l}\text { Not involved in substrate } \\
\text { binding or catalysis }\end{array}$ & No. \\
\hline Y103 & $\begin{array}{c}\text { Facilitates } \gamma \text {-elimination- } \\
\text { specific catalytic step }\end{array}$ & $\begin{array}{l}\text { Not involved in substrate } \\
\text { binding or catalysis }\end{array}$ & $\begin{array}{c}\text { Not involved in substrate } \\
\text { binding or catalysis. }\end{array}$ & $\begin{array}{c}\text { Determinant of } \gamma \text { - } \\
\text { elimination RS (CGL). }\end{array}$ \\
\hline $\mathbf{R 1 0 8}$ & $\begin{array}{l}\text { Binds L-Cth distal carboxylate, } \\
\text { affects active-site architecture. }\end{array}$ & $\begin{array}{c}\text { Not involved in substrate } \\
\text { binding or catalysis }\end{array}$ & $\begin{array}{c}\text { Binds L-OSHS distal } \\
\text { carboxylate }\end{array}$ & $\begin{array}{l}\text { Partial determinant of RS } \\
\text { (CGS and CGL). }\end{array}$ \\
\hline $\mathbf{N} 232$ & $\begin{array}{l}\text { Binds L-Cth distal carboxylate, } \\
\text { may impact PLP binding. }\end{array}$ & Binds L-Cth distal carboxylate & $\begin{array}{c}\text { Not involved in substrate } \\
\text { binding or catalysis }\end{array}$ & $\begin{array}{c}\text { Determinant of SS (CBL } \\
\text { and CGL). } \\
\end{array}$ \\
\hline $\mathbf{E 3 3 3}$ & $\begin{array}{l}\text { Binds L-Cth distal amino, } \\
\text { important for charge distribution }\end{array}$ & $\begin{array}{c}\text { Not involved in substrate } \\
\text { binding or catalysis }\end{array}$ & $\begin{array}{l}\text { Partial determinant of reaction } \\
\text { specificity }\end{array}$ & $\begin{array}{l}\text { Determinant of SS (CGL) } \\
\text { and RS (CGS and CGL). }\end{array}$ \\
\hline S334 & Tethers/guides catalytic lysine & Tethers/guides catalytic lysine & Tethers/guides catalytic lysine & $\begin{array}{l}\text { Determinant of RS (CGS, } \\
\text { CBL, and CGL). }\end{array}$ \\
\hline $\mathbf{R 3 6 9}$ & Binds L-Cth $\alpha$-carboxylate & Binds L-Cth $\alpha$-carboxylate & Binds L-OSHS $\alpha$-carboxylate & No. \\
\hline
\end{tabular}

${ }^{2}$ Farsi et al., 2009; ${ }^{b}$ Lodha et al., 2010; ' Codha and Aitken, 2011; Jaworski et al., 2012. 
than that of the DTNB-detected hydrolysis of L-Cth, although this may reflect a difference in the substrate and/or reaction specificities of hCGL (Chiku et al., 2009).

The substrate inhibition of pyruvate production observed for the yCGL-catalyzed hydrolysis of L-Cys (Figure 3.3A) suggests that a second molecule of L-Cys may bind to the active site, thereby inhibiting the production of pyruvate from L-Cys (Chiku et al., 2009). This does not necessarily signify that enzymatic catalysis is inhibited; rather, it likely indicates that an alternate reaction is enabled upon binding of a second molecule of L-Cys. Attack of the thiolate moiety of the second L-Cys molecule on the aminoacrylate intermediate formed by $\beta$-elimination of hydrogen sulfide from the first would, via a $\beta$ replacement reaction, produce lanthionine (Figure 1.10B) and result in the observed decrease in activity when measuring pyruvate production. The plausibility of this scenario is supported by the low reaction specificity of yCGL and the observation that the native L-Cth substrate is more than twice the size of L-Cys, suggesting that two L-Cys molecules can be accommodated in the yCGL active site, particularly given the pseudosymmetric nature of L-Cth. Indeed, similar concentration-dependent reaction specificity has been reported for hCGL, where the main contributing reaction to hydrogen sulfide production switches from $\beta$-elimination to $\beta$-replacement, with the concomitant production of lanthionine, as L-Cys concentration increases (Figure 1.10B; Chiku et al., 2009).

The low substrate and reaction specificity of yCGL is further exemplified by the ability of the wild-type enzyme to catalyze the $\beta$-elimination of L-OAS (Table 3.2). While the $K_{\mathrm{m}}{ }^{\mathrm{LOAS}}$ is only 3 -fold higher than $K_{\mathrm{m}}{ }^{\mathrm{L} C \mathrm{Cth}}$, the catalytic efficiency of this reaction is 560 -fold lower than that of the physiological hydrolysis of L-Cth, largely due to a 155 - 
fold decrease in $k_{\text {cat }}{ }^{\text {LOAS }}$, indicating that while the active site of yCGL easily accommodates the L-OAS substrate, its architecture is such that L-OAS is bound in a conformation that does not favour the $\beta$-elimination of the distal acetate group of this alternative substrate.

The substrates L-Cys/L-OAS and L-Hcys are useful probes of reaction specificity as they are specific for $\beta$ - and $\gamma$-elimination, respectively (Figure 1.10). The wild-type yCGL enzyme is not saturated by $100 \mathrm{mM} \mathrm{L-Hcys,} \mathrm{and} \mathrm{catalyzes} \mathrm{the} \mathrm{hydrolysis} \mathrm{of} \mathrm{this}$ substrate with a catalytic efficiency 77,000-fold lower than that of L-Cth (Table 3.2). This indicates that, similar to L-OAS, the yCGL active-site does not accommodate the binding of L-Hcys in a catalytically productive manner. The extra methylene carbon of the L-Hcys side chain, compared to L-Cys, adds a greater degree of conformational flexibility to the substrate molecule, and the absence of the distal carboxylate and amino groups of L-Cth may preclude the appropriate positioning of the thiol group of L-Hcys in the active-site. The combination of increased conformational flexibility and inappropriate orientation of the substrate, via positioning of the distal portion, could result in the observed inefficiency with which wild-type yCGL hydrolyzes L-Hcys and L-OAS. This suggests that tethering of the substrate through the interaction of the distal end with active-site residues is necessary to properly position the substrate for efficient catalysis. Another cause of the reduced $k_{\text {cat }} / K_{\mathrm{m}}{ }^{\mathrm{L} H c y s}$ that cannot be discounted is the potential of yCGL to preferentially catalyze an alternate reaction with L-Hcys, such as a $\gamma$-replacement between two L-Hcys molecules to produce homolanthionine (Figure 1.10B), thereby precluding the formation of the $\alpha$-ketobutyrate product detected in the HO-HxoDH assay. This possibility is illustrated by the report that $V_{\max }$ of the human CGL enzyme for the 
production of homolanthionine by condensation of two L-Hcys molecules, via a $\gamma$ replacment mechanism, is $\sim 5$-fold greater than the $\gamma$-elimination of L-Hcys, which produces $\alpha$-ketobutyrate (Chiku et al., 2009).

The bell-shaped $\mathrm{pH}$ dependence of the $k_{\mathrm{cat}} / K_{\mathrm{m}}^{\mathrm{L}-\mathrm{Cth}}$ of yCGL has a $\mathrm{pH}$ optimum of $7.6-8$, in agreement with the reported optima of $7-8$ for yCGL (Farsi et al., 2009) and 8.2 for the human enzyme (Steegborn et al., 1999). The $\mathrm{p} K_{\mathrm{a}}$ values of the acidic and basic limbs of the $k_{c a l} / K_{\mathrm{m}}{ }^{\mathrm{L}-\mathrm{Cth}} \mathrm{pH}$ profile are $8.1 \pm 0.4$ and $7.1 \pm 0.4$, respectively (Table 3.4). The acidic limb $\mathrm{p} K_{\mathrm{a}}$ value is within experimental error of that of the eCBL $k_{\mathrm{cat}} / K_{\mathrm{m}}{ }^{\mathrm{L}-\mathrm{Cth}}$ versus $\mathrm{pH}$ profile $\left(\mathrm{p} K_{\mathrm{a} 1}=8.28 \pm 0.06\right)$ (Lodha and Aitken, 2011) and may correspond to the $\alpha-\mathrm{NH}_{3}$ group of $\mathrm{L}-\mathrm{Cth}\left(\mathrm{p} K_{\mathrm{a}}=8.54 \pm 0.01\right)$ (Aitken and Kirsch, 2003), which must be in the neutral $\left(\mathrm{NH}_{2}\right)$ state in both enzymes for nucleophilic attack on the internal aldimine nitrogen in order for transaldimination to occur. The basic limb of the catalytic efficiency versus $\mathrm{pH}$ profile corresponds to a group, likely an active-site residue, which must be protonated to enable substrate binding or a step of catalysis. The catalytic efficiency of yCGL is approximately two orders of magnitude lower than that of eCBL (Farsi et al., 2009; Lodha and Aitken, 2011). This difference is the result of the $\mathrm{p} K_{\mathrm{a}}$ of the acidic limb $\left(\mathrm{p} K_{\mathrm{a} 1}=8.1 \pm 0.4\right)$ exceeding that of the basic limb $\left(\mathrm{p} K_{\mathrm{a} 2}=7.1 \pm 0.4\right)$, such that the $\mathrm{pH}$ profile and optimum are correspondingly sharp and narrow and the observed catalytic efficiency of yCGL is 10 -fold lower than that theoretically possible because the titrating groups represented by $\mathrm{p} K_{\mathrm{a} 1}$ and $\mathrm{p} K_{\mathrm{a} 2}$ cannot be simultaneously fully deprotonated and protonated, respectively. In contrast $\mathrm{p} K_{\mathrm{a} 1}\left(\mathrm{p} K_{\mathrm{a} 1}=8.28 \pm 0.06\right)$ of the eCBL catalytic efficiency versus $\mathrm{pH}$ profile is $1.9 \mathrm{pH}$ units lower than $\mathrm{p} K_{\mathrm{a} 2}\left(\mathrm{p} K_{\mathrm{a} 2}=10.20 \pm 0.06\right)$, 
thereby allowing the enzyme to achieve $\sim 50 \%$ of the theoretical maximal catalytic efficiency at the pH optimum (Lodha and Aitken, 2011).

Interestingly, these differences in the basic limb $\mathrm{p} K_{\mathrm{a}}$ values between $\mathrm{yCGL}$ and $\mathrm{eCBL}$ are mirrored in the $\mathrm{p} K_{\mathrm{a}}$ values of the internal aldimine of these enzymes $(8.84 \pm$ 0.07 and $10.81 \pm 0.08$ respectively), determined by spectrophotometric titration (Figure 3.5). This suggests that the basic limb $\mathrm{p} K_{\mathrm{a}}$ values of $y C G L$ and $\mathrm{eCBL}$ may be assigned to the internal aldimines of these enzymes (which must be protonated in both cases for transaldimination), and suggest that the unique $\mathrm{pH}$ profiles of yCGL and $\mathrm{eCBL}$ are a result of differences in the $\mathrm{p} K_{\mathrm{a}}$ of their respective internal aldimines. The internal aldimine $\mathrm{p} K_{\mathrm{a}}$ of fold type I enzymes varies, as exemplified by the aldimine $\mathrm{p} K_{\mathrm{a}}$ values of $6.9,7.4$, and 8.1 for E. coli AAT, tryptophan indole-lyase, and Salmonella typhymurium OASS respectively (Goldberg et al., 1991; Phillips et al., 1991; Cook et al., 1992). The $\sim 2 \mathrm{pH}$ unit difference in aldimine $\mathrm{p} K_{\mathrm{a}}$ values of $\mathrm{yCGL}$ and $\mathrm{eCBL}$ could be a function related to slight differences in the acidity of the $\alpha$-proton of L-Cth, depending on whether it is bound in the orientation for $\beta$ - or $\gamma$-elimination. When $\mathrm{L}-\mathrm{Cth}$ is bound in the orientation required for $\gamma$-elimination, the extra methylene carbon between $C_{\alpha}$ and $S_{\gamma}$, compared to when when L-Cth is bound in orientation for $\beta$-elimination, could diminish the electron-withdrawing effects of $S_{\gamma}$ on $C_{\alpha}$, making the $\alpha$-proton less acidic and therefore requiring slightly greater nucleophilicity to abstract in a $\gamma$-elimination. The decreased $\mathrm{p} K_{\mathrm{a}}$ of the basic limb/aldimine of $\mathrm{yCGL}$ could reflect the need for the lower $\mathrm{p} K_{\mathrm{a}}$ of $\mathrm{K} 203$ which would be required to abstract the proton from $\mathrm{C}_{\alpha}$ for $\gamma$-elimination. Thus the dissimilar $\mathrm{p} K_{\mathrm{a}}$ values of the internal aldimine of yCGL and $\mathrm{eCBL}$ may indicate differences in the active-site environment, in proximity to the catalytic base, of these two 
enzymes and may be a factor that distinguishes the reaction specificies of these two enzymes.

The formation of a shoulder at $330 \mathrm{~nm}$ in the absorbance spectrum of wild-type yCGL following reaction for $120 \mathrm{~min}$ with $3 \mathrm{mM} \mathrm{L-Cth}$ (Figure $3.6 \mathrm{~B}$, inset) is indicative of formation of the ketimine derivate resulting from protonation of C4' of the cofactor during $\gamma$-elimination (Figure 1.9; Toney and Kirsch, 1991). It is also similar to the 330$\mathrm{nm}$ peak corresponding to the formation of a ketimine intermediate which accompanies the loss of absorbance at $427 \mathrm{~nm}$ observed for human CGL after reaction with the inhibitor AVG (Steegbom et al., 1999). In contrast, the lack of a defined 330-nm absorbance and absence of an isobestic point after reaction of yCGL for $120 \mathrm{~min}$ with $100 \mathrm{mM}$ L-alanine (Figure 3.6A, inset) suggests an inability to protonate $\mathrm{C} 4$ ' to the same extent after external aldimine formation with this substrate, and/or that formation of the species absorbing at $330 \mathrm{~nm}$ does not precede the rate-determining step in reaction of yCGL with L-alanine.

\subsection{The $R 51 A / K$ variants.}

Arginine residues R51 and R108 and the glutamate at position 333 of yCGL have been proposed to interact with the distal carboxylate and distal amino groups, respectively, of L-Cth (Figure 1.5; Messerschmidt et al., 2003). The yCGL-R51A/K variants cannot be saturated within the solubility limit of L-Cth (Table 3.5). This indicates that increases in $K_{\mathrm{m}}{ }^{\mathrm{L} C \mathrm{Cth}}$ are a factor in the observed 32,800 and 340 -fold decreases in the catalytic efficiency of the alanine and lysine replacement variants of R51 (Table 3.5), 
thereby demonstrating an important role for this residue in interacting with the L-Cth substrate. Replacement of R51 with lysine, which maintains the charge and part of the hydrogen-bonding capacity of the native arginine residue at this position, but reduces the length of the side chain by $\sim 1 \AA$, results in a 340 -fold decrease in catalytic efficiency (Table 3.5). This observation highlights the crucial role of this residue, which also donates a pair of hydrogen bonds to the phosphate group of the PLP cofactor, in maintaining proper active-site architecture for productive substrate binding. In all fold type I enzymes of the forward and reverse transsulfuration pathways, the residue corresponding to yCGL-R51 is conserved as arginine, as exemplified by eCGS-R48 and eCBL-R58, which play similar roles in the active sites of these enzymes (Messerschmidt et al., 2003; Clausen et al., 1997; Clausen et al., 1998; Lodha et al., 2010; Jaworski et al., 2012).

The 420 -fold reduction in $k_{\text {cat }} / K_{\mathrm{m}}{ }^{\mathrm{L}-\mathrm{OAS}}$ for $\mathrm{R} 51 \mathrm{~K}$ and the inability to saturate $\mathrm{R} 51 \mathrm{~K}$ within the solubility limit of this substrate (Table 3.5), which lacks the distal amino group of L-Cth but maintains a carbonyl moiety in the acetate portion of the molecule, corroborates a role for residue R51 of yCGL in binding the distal carboxylate of L-Cth, similar to the corresponding arginine residues of eCGS and eCBL in binding the distal carboxylate groups of L-OSHS and L-Cth, respectively (Lodha et al., 2010; Jaworski et al., 2012). However, the R51A/K-catalyzed hydrolysis of L-Cys and L-Hcys, which both lack the distal carboxylate of L-Cth, was undetectable, suggesting that the lack of activity observed with these substrates may be due to incorrect positioning of PLP. The inability of the $\varepsilon$-amino group of lysine (R51K) to fulfil all of the hydrogen-bonding capabilities of the guanidinium moiety of the arginine side chain of R51 could result in altered 
positioning of the distal carboxylate group in the context of yCGL-R51K. This could result in altered reaction specificity, similar to the increased racemization and $\beta$ decarboxylation activity observed for the R292K substitution of the corresponding AATR292, which is responsible for binding the distal carboxylate group of dicarboxylic substrates (Vacca et al., 1997). In this way, R51 likely plays a dual role in the active-site of yCGL by positioning the cofactor through a hydrogen bond with the PLP phosphate as well as anchoring the distal carboxylate of L-Cth to place the substrate optimally for catalysis. These roles of R51 are likely related, and the position of the phosphate group of the cofactor may partially determine the position of the distal carboxylate group of the substrate, via hydrogen bond(s) formed to the guanidinium side chain of R51 (Figure $1.5)$.

The appearance of a 330-nm peak indicative of protonation of $\mathrm{C} 4$ ' after reaction with L-alanine (Figure 1.11), which is not a substrate for the $\gamma$-elimination reaction, in the absorbance spectrum of the R51A/K variants, which the wild type lacks (Figure 3.8A), suggests that absence of the tethering effect of the R51 side chain on the PLP phosphate results in a loss of specificity, likely through increased mobility of the cofactor. This interaction appears to affect the reaction specificity of yCGL more than that of eCGS, which also catalyzes a $\gamma$-elimination reaction, as the corresponding eCGS-R $48 \mathrm{~A} / \mathrm{K}$ variants exhibit spectral changes identical to the wild-type eCGS after reaction with alanine (Jaworski et al., 2012). Thus, in addition to supporting the substrate-binding hypothesis for R51, a function of this residue in affecting the specificity of yCGL, likely via hydrogen bonding to maintain the relative positions of the substrate and cofactor, is elucidated. 


\subsection{The RI08A/K variants.}

Replacement of residue R108 with alanine or lysine results in 240 and 200 -fold decreases, respectively, in $k_{\text {cat }} / K_{\mathrm{m}}{ }^{\mathrm{L}-\mathrm{Cth}}$ (Table 3.5). Messerschmidt et al. (2003) proposed a role for this residue in substrate binding, via interaction with the distal carboxylate group of L-Cth (Figure 1.5). The decrease in catalytic efficiency of the R108A variant results from the combination of a 31-fold decrease and 8-fold increase in $k_{\text {cat }}$ and $K_{\mathrm{m}}{ }^{\mathrm{L}-\mathrm{Cth}}$, respectively (Table 3.5 ). This suggests that, in addition to removing the hydrogenbonding capacity of this residue, an alanine at position 108 sufficiently alters the activesite architecture or positioning of the substrate such that the ability of the L-Cth substrate to bind in a catalytically productive orientation is decreased. Similarly, the equal, $\sim 15-$ fold changes in $k_{\text {cat }}$ and $K_{\mathrm{m}}^{\text {L-Cth }}$ that underlie the 200 -fold reduction in the catalytic efficiency of yCGL-R108K indicate that the $\sim 1-\AA$ shorter side chain of lysine is insufficient to allow optimal substrate binding. Furthermore, L-OAS hydrolysis activity is not detectable for the alanine and lysine substitution variants of residue R108, signifying that this residue plays a similar role in anchoring the carbonyl oxygen of the L-OAS acetate group to orient the substrate for catalysis. The absence of an increase in $K_{\mathrm{m}}{ }^{\mathrm{L} C y s}$, compared the 8-15-fold increases in $K_{\mathrm{m}}{ }^{\mathrm{L} C \mathrm{Cth}}$ observed for the $\mathrm{R} 108 \mathrm{~A} / \mathrm{K}$ variants, suggests that R108 is not positioned proximal to the smaller L-Cys molecule when the latter is bound in aldimine linkage to the cofactor, occupying the site of the proximal portion of the L-Cth binding site, thereby providing further evidence for interaction of R108 with the distal carboxylate of L-Cth. This is supported by the observed 5-fold decrease in $K_{\mathrm{i}}^{\mathrm{L}-\mathrm{Cys}}$ of R108K (Table 3.5) as shortening of the basic side chain at position R108 improves the positioning of a second L-Cys molecule, via interaction with the carboxylate moiety of L- 
Cys to facilitate the $\beta$-replacement reaction that produces lanthionine, which is one carbon smaller than the native L-Cth substrate of yCGL. Thus, the hypothesized role of this residue in substrate-binding is supported.

The 200- to 240-fold decreases in catalytic efficiency of L-Cth hydrolysis observed for the yCGL-R108A/K variants (Table 3.5) are comparable to the 55- to 140 fold $k_{\text {cat }} / K_{\mathrm{m}}{ }^{\text {L-OSHS }}$ decreases reported for the $\gamma$-elimination of L-OSHS by the corresponding eCGS-R106A/K variants (Jaworski et al., 2012). However, the decreases in catalytic efficiency of the yCGL variants are due to approximately equal contributions of 15-30-fold decreases and 8-13-fold increases in $k_{\text {cat }}$ and $K_{\mathrm{m}}{ }^{\text {L-Cth }}$, respectively (Table 3.5), while those of the corresponding eCGS variants are dominated by 16-20-fold increases in $K_{\mathrm{m}}^{\text {L-OSHS }}$ (Jaworski et al., 2012). This suggests that while this arginine residue is involved in substrate tethering in both enzymes it plays an additional role in yCGL in productively orienting the substrate within the active site. The guanidinium group of R108 is $\sim 3 \AA$ away from the side chain of $\mathrm{R} 51$ in the context of the yCGL active site (Figure 1.5), thereby positioning this residue to bind the distal carboxylate group of the substrate in concert with R51. In contrast with yCGL-R108 and eCGS-R106, the corresponding residue of eCBL is an aspartate (D116) that is not involved in substrate binding (Lodha et al., 2010). This suggests that an arginine at this position appears to be necessary for orienting the substrate in enzymes that carry out a $\gamma$-elimination. This difference is of note as the residue at position 108 (yCGL numbering) interacts with the distal portion of L-Cth only in yCGL, and thus may act as a determinant of reaction specificity for yCGL and eCBL, which catalyze the $\gamma$ and $\beta$-elimination, respectively, of this pseudosymmetric substrate. 


\subsection{The N232A variant.}

Residue N232, conserved as N227 in eCGS, was targeted for site-directed substitution because the corresponding eCBL-Y238 was shown to interact with the distal carboxylate of L-Cth (Lodha and Aitken, 2011). The 70-fold reduction in $k_{\text {cat }} / K_{\mathrm{m}}^{\text {L-Cth }}$ of the yCGL-N232A variant is dominated by a 21 -fold increase in $K_{\mathrm{m}}{ }^{\mathrm{L}-\mathrm{Cth}}$, as $k_{\text {cat }}$ is decreased only 3 -fold, compared to the wild-type enzyme (Table 3.5 ). This is comparable to the 15 -fold increase in $K_{\mathrm{m}}^{\mathrm{L}-\mathrm{Cth}}$ observed for eCBL-Y238F (Lodha and Aitken, 2011), which interacts with the distal carboxylate of $\mathrm{L}-\mathrm{C}$ th, thus supporting a similar role for this residue in $y C G L$ in interacting with the distal portion of the substrate, as proposed in the hypotheses of this study.

The 270 -fold decrease in $k_{\text {cat }} / K_{\mathrm{m}}{ }^{\mathrm{LOAS}}$ of N232A (Table 3.5), which is not saturated by $200 \mathrm{mM} \mathrm{L-OAS}$, also points to a role for this residue in interacting with distal carboxylate of the substrate, as L-OAS lacks the distal amino moiety, but maintains a carbonyl functional group in the acetate portion of molecule, similar to the distal carboxylate of L-Cth. The $\mathrm{N} 232 \mathrm{~A}$ and $\mathrm{R} 51 \mathrm{~K}$ variants of yCGL are unique in their inability to be saturated by L-OAS at $200 \mathrm{mM}$ (Table 3.5 ). The $3.5-\AA$ distance between the side chains of these residues, allowing their hydrogen-bond-mediated interaction (Figure 1.5; Messerschmidt et al., 2003), as well as the similar changes in kinetic parameters for the $\mathrm{N} 232 \mathrm{~A}$ and $\mathrm{R} 51 \mathrm{~K}$ variants suggest a common role for these residues in interacting with the distal carboxylate to tether and orient the substrate. The increased $K_{\mathrm{m}}$ and decreased $k_{\text {cat }} / K_{\mathrm{m}}$ for the hydrolysis of L-Cth and L-OAS, respectively, may also be attributable to decreased tethering of PLP, or an additive effect of loss of R51-mediated PLP- and substrate-binding interactions. However, an 11-fold increase in $K_{\mathrm{m}}{ }^{\mathrm{L}-\mathrm{Cys}}$ was also 
observed for the N232A-catalyzed hydrolysis of L-Cys (Table 3.5), which lacks the distal carboxylate and amino groups of L-Cth, suggesting that the observed increase in $K_{\mathrm{m}}{ }^{\text {L-Cys }}$ may be a result of a change in the architecture of the active site resulting from loss of the R51-mediated, indirect interaction of N232 with the PLP phosphate.

The corresponding residue $\mathrm{Y} 238$ in $\mathrm{eCBL}$ has similarly been shown to interact with the distal carboxylate of L-Cth (Lodha and Aitken, 2011), but the <4-fold changes in kinetic parameters for the corresponding eCGS-N227A variant demonstrate that this residue is not important for substrate binding or catalysis in eCGS (Jaworski et al., 2012). Thus the identity of this residue does not affect $\beta$ - or $\gamma$-elimination reaction specificity; however, it plays a necessary role in facilitating the binding of L-Cth in both eCBL and yCGL. The increased hydrogen-bonding capacity of asparagine compared to tyrosine allows this residue to play an additional role in yCGL in positioning the PLP cofactor via R51.

In patients with cystathioninuria, mutations have identified affecting the residues in hCGL corresponding to yCGL-R51 and Q231, immediately adjacent to N232 (Kraus et al., 2009). This indicates that the abilities of R51 and N232 to bind the substrate and cofactor are essential for a functional CGL enzyme, and that disruption of these residues' functions due to mutation may underlie the phenotype of cystathioninuria.

\subsection{The $R 369 A / K$ variants.}

The $\alpha$-carboxylate of $\mathrm{L}$-Cth has been proposed to be tethered in the yCGL active site by interactions with R369 (Figure 1.5; Messerschmidt et al., 2003). The 17,500-fold 
and $\sim 15,000$-fold reductions in catalytic efficiency of the alanine and lysine substitutions, respectively, of this residue (Table 3.5), as well as the inability of these variants to be saturated within the solubility limit of L-Cth, confirms the role of this residue in binding the $\alpha$-carboxylate of the substrate. This is additionally supported by the lack of detectable activity of these variants for the hydrolysis of the alternative substrates L-Cys, L-Hcys, and L-OAS, which also possess an a-carboxylate group, but are limited their ability to form hydrogen-bonding interactions via their distal portions, compared to L-Cth. The corresponding eCGS-R361 and eCBL-R372 have also been shown to be essential in binding the $\alpha$-carboxylate groups of these enzymes' respective substrates, L-OSHS and LCth (Jaworski et al., 2012; Lodha et al., 2010). The essential function of R369 in binding the $\alpha$-carboxylate of amino acid substrates is mirrored in the conservation of the identity and function of this residue across the $\gamma$-subfamily (Messerschmidt et al., 2003; Lodha et al., 2010; Jaworski et al., 2012) and in the broader fold type I of PLP-dependent enzymes (Vacca et al., 1997).

\subsection{The $S 52 A / R$ variants.}

The residue at position 52 varies considerably among the members of the $\gamma$ subfamily of fold type I of PLP dependent enzymes, for which 14 crystal structures exist (Aitken et al., 2011). A serine at this position appears in the yeast and human CGL enzymes, as well as in CBL from Arabidopsis thaliana, while the corresponding residue of Nicotiana tabacum CGS and of eCBL and eCGS is a tyrosine and arginine, respectively (Messerschmidt et al., 2003). Although roles in substrate binding were 
proposed for both eCGS-R49 and eCBL-R59 on the basis of the crystal structures of these enzymes, replacement of these residues with alanine has a negligible effect on the kinetic parameters (Lodha et al., 2010; Jaworski et al., 2012). The difference in amino acid identity at this position in yCGL compared to eCGS and eCBL suggested that this residue may play a role in determining substrate/reaction specificity. Therefore, the yCGL-S52R variant, introducing the conserved arginine of eCGS and eCBL, was constructed. The minor 8-fold $K_{\mathrm{m}}^{\text {LCth }}$ increase observed upon replacement of S52 with arginine (Table 3.5 ) is likely caused by changes in active-site architecture due to the introduction of the bulkier arginine residue, resulting in less productive L-Cth binding, and not due to alterations of necessary interactions, as the side chain of this residue in the crystal structure is oriented away from the substrate-binding pocket (Figure 1.6) and appears not to form any salt bridges with other active-site residues in the structure of yCGL (Figure 1.5). Substitution of residue S52 with alanine or arginine resulted in unexpected 18 -fold increases in the $K_{\mathrm{m}}^{\mathrm{L}-\mathrm{C} y \mathrm{~s}}$ of both variants compared to the wild type (Table 3.5), suggesting that removal of the hydroxyl group of this residue or replacement with a bulky side chain may alter active-site architecture to hinder L-Cys binding or impede entrance of the substrate into the active-site. However, if this residue affects the substrate or reaction specificity of yCGL it is only in a minor way, as the kinetic parameters of the S52A/R variants are within 2.5 -fold of the wild type for the hydrolysis of both L-Cth and L-OAS (Table 3.5). Thus the hypothesized role of this residue in binding the substrate and affecting specificity is not supported, as the kinetic data indicate that, similar to the corresponding arginine residues of eCGS (R49) and eCBL (R59), the 
serine residue at position 52 of yCGL is not involved in catalysis or substrate binding of the physiological substrate.

\subsection{The Y49F variant.}

The crystal structure of yCGL suggests that residue Y49 is involved in tethering the PLP cofactor at the active site via a $3.0-\AA$ hydrogen bond between the hydroxyl group of the tyrosine side chain and an oxygen atom of the PLP phosphate moiety (Figure 1.5; Messerschmidt et al., 2003). The $\sim 750$-fold reduction in catalytic efficiency of L-Cth hydrolysis by the yCGL-Y49F variant is dominated by a 190 -fold decrease in $k_{\text {cat }}$, as the $K_{\mathrm{m}}^{\text {L-Cth }}$ of this mutant is increased only 4 -fold (Table 3.6), suggesting a role for this

residue in catalysis and/or maintenance of active-site architecture. The minor $K_{\mathrm{m}}{ }^{\mathrm{L}-\mathrm{Cth}}$ increase of the Y49F mutant suggests that the hydrogen bonding ability of this residue is not essential for proper cofactor or substrate binding, in contrast with the hypothesized role of this residue and with the roles of the corresponding eCGS-Y46 and eCBL-Y56 residues, for which the $K_{\mathrm{m}}$ values of their L-OSHS and L-Cth substrates are increased $\sim 20$-fold, respectively. This is likely due to an increase in $K_{d}{ }^{\text {PLP }}$ as a result of loss of the hydrogen bond between the tyrosine side chain and the PLP phosphate (Lodha and Aitken, 2011; Jaworski et al., 2012). In the context of yCGL-Y49F, the remaining hydrogen bonding contributions of the other active-site residues involved in binding the substrate and anchoring the cofactor (Figure 1.5) are sufficient to maintain adequate PLP saturation, such that the role of $\mathrm{Y} 49$ is minor compared to eCGS and eCBL. While this residue may not be required for substrate binding, the 190 -fold decrease in $k_{\text {cat }}$ observed 
for the Y49F variant (Table 3.6) suggests a role in catalysis. Based on the the structure of the eCBL-AVG complex, residues $Y 49$ and $S 334$ are expected to tether the $\varepsilon$-amino group of the catalytic base, when the substrate replaces K203 in covalent linkage with PLP (Figure 1.5) (Clausen et al., 1997).

Substitution of Y49 with phenylalanine also results in a reduction in $k_{\text {cat }}{ }^{\text {LCys }}$ (Table 3.6). Although this 27-fold decrease is the largest reduction in this parameter of the site-directed variants tested, it is an order of magnitude smaller than the 190 -fold reduction in $k_{\text {cat }}^{\text {L.Cth }}$ decrease observed for the same variant (Table 3.6). While no crystal structures exist of yCGL in complex with an active-site ligand, the positioning of Y49 within the active site, as well as comparison to the eCBL-AVG structure (Clausen et al., 1997; Messerschmidt et al., 2003) suggests that the side-chain hydroxyl moiety may be situated in proximity to $C_{\beta}$ of the substrate (Figure 1.5). This raises the possibility that Y49 may guide the catalytic lysine to $C_{\beta}$ to facilitate the second proton abstraction necessary for $\gamma$-elimination. However, similar to the corresponding eCBL-Y56F variant, the minor alterations in $\mathrm{pH}$ dependence of the specific activity of Y49F (Figure 3.11A) indicate that this residue is not involved directly in proton transfer (Lodha and Aitken, 2011). Therefore, the reductions in $k_{\text {cat }}{ }^{L C C t h}$ and $k_{\text {cat }}{ }^{{ }^{L C C y s}}$ observed for Y49F indicate that the role of this residue is not specific to $\gamma$-elimination, but demonstrate that it is required for maintenance of catalytically productive active-site architecture or facilitating a catalytic step common to both $\beta$ - and $\gamma$-elimination, such as guiding the catalytic lysine to the sulfur atom for protonation to facilitate the leaving group. However, the kinetic parameters for L-OAS hydrolysis by Y49F are altered by less than 3-fold from the wild type (Table 3.6), indicating that the role Y49 plays in the $\gamma$ - and $\beta$-elimination of L-Cth 
and L-Cys, respectively, is not necessary for the $\beta$-elimination of L-OAS. This could be due to the increased electronegativity of the L-OAS $\mathrm{O}_{\beta}$ atom, compared to the $\mathrm{S}_{\gamma}$ atom of L-Cth, making acetate a better leaving group than the L-Cys and $\mathrm{H}_{2} \mathrm{~S}$ leaving groups of LCth and L-Cys hydrolysis, respectively, which could eliminate the need for the electronegative hydroxyl group of Y49 to attract the catalytic lysine for protonation of the leaving group.

While substitution of this conserved tyrosine residue in yCGL with phenylalanine results in a 190-fold decreased $k_{\text {cat }}^{\text {LCCth }}$ (Table 3.6) and near-wild-type parameters for LOAS hydrolysis (Table 3.6), the corresponding Y56F variant in eCBL displays only a minor, 6-fold reduction in $k_{\text {cat }}^{\text {L-Cth }}$ and does not saturate with L-OAS (Lodha and Aitken, 2011). The 190 -fold decrease in $k_{\text {cat }}^{\text {L-Cth }}$ observed for the yCGL-Y49F variant is unique, as phenylalanine substitutions of corresponding tyrosine residues of the transsulfuration enzymes resulted in $k_{\text {cat }}$ decreases of only 6-fold for eCBL-Y56F (Lodha and Aitken, 2011), and 20-fold for the $\gamma$-replacement activity of eCGS-Y46F (Jaworski et al., 2012). However, the $k_{\text {cat }}$ of eCGS-Y46F for the $\gamma$-elimination of L-OSHS, the mechanism of which is proposed to be the same as for the yCGL-catalyzed $\gamma$-elimination of L-Cth (Brzovic et al., 1990; Messerschmidt et al., 2003), was unchanged from the wild-type (Jaworski et al., 2012). Among the broader fold type I family of PLP-dependent enzymes such as aminolevulinate synthase (ALAS) and AAT, the conserved tyrosine residue corresponding to yCGL-Y49 has similar roles proposed in tethering the phosphate group of the PLP cofactor (Tan et al., 1998). In murine erythroid ALAS the hydrogen-bonding contribution of the corresponding tyrosine residue is a factor in cofactor-enzyme affinity (Tan et al., 1998), while in $E$. coli AAT it plays less of a role in substrate/cofactor 
binding, as indicated by the minor 3-fold increase in $K_{\mathrm{m}}$ of the Y70F mutant, and is more involved in modulation of the catalytic activity (Toney and Kirsch, 1991). The unique and varying roles of this tyrosine, conserved among fold type I PLP-dependent enzymes, highlight how subtle differences in positioning of active-site residues can alter substrate and reaction specificities.

The modest increase in absorbance at $330 \mathrm{~nm}$ in the spectrum of the Y49F variant after reaction with $\mathrm{L}-\mathrm{Cth}$, as well as the lack of isobestic point around $370 \mathrm{~nm}$ compared to the wild type (Figure 3.13B), suggest that the Y49F variant retains some ability to form the ketimine intermediate, but that the rate-determining step is shifted and/or additional "off-path" reaction intermediates are formed. The appearance of a $330-\mathrm{nm}$ peak for yCGL-Y49F, suggestive of protonation of C4' due to formation of PMP as a result of transamination, after reaction with L-alanine (Figure 3.13A), which can undergo racemization or transamination but not $\beta$ or $\gamma$ side-chain rearrangements, is distinct from wild type yCGL and from the corresponding eCBL-Y56F variant (Lodha and Aitken, 2011; Cellini et al., 2007). Similar spectral changes, following reaction with L-alanine, are observed for the R51A/K and S334A variants of yCGL (Figures 3.8A and 3.13A) and eCGS-Y46F (Jaworski et al., 2012). This indicates that, in the context of the active sites of yCGL and eCGS, loss of the hydrogen bond between the yCGL-Y49 hydroxyl and the PLP phosphate and/or the tethering interaction between Y49 and the catalytic lysine results in spectral changes similar to loss of the PLP-R51 and the S334-K203 tethering interactions. This may indicate that repositioning of the cofactor or the catalytic base by alteration of the network that maintains the relative positions of $\mathrm{K} 203$, the catalytic base, and the anchoring phosphate moiety of the cofactor results in altered reaction specificity. 


\subsection{The Y103F variant.}

Several key mechanistic and structural roles have been proposed for residue Y103, including activation of the incoming substrate for transaldimination by proton abstraction, facilitation of the release of the L-Cys product, and maintaining the cofactor in a catalytically productive conformation within the active site via $\pi$-stacking interactions between the aromatic rings of the tyrosine side chain and the pyridine ring of PLP (Messerschmidt et al., 2003). This residue is conserved as a tyrosine in CGS and $\mathrm{CBL}$, and similar roles have been proposed, based on available crystallographic structures (Clausen et al., 1998; Clausen et al., 1996; Messerschmidt et al., 2003). However, kinetic analyses of phenylalanine substitutions of this residue do not support a role in proton transfer or catalysis in these enzymes (Lodha and Aitken, 2011; Jaworski et al., 2012). Additionally, it is the presence of an aromatic amino acid, and not specifically a tyrosine that is conserved at this position in fold type I enzymes (Hayashi, 1995), suggesting that the $\pi$-stacking interactions with the cofactor, and not proton transfer, is the common role of this residue. However, the 420 -fold decrease in $k_{\text {cat }}$ for L-Cth hydrolysis of the yCGLY103F mutant (Table 3.6) lies in sharp contrast to the near-wild-type $k_{\text {cat }}$ values reported for corresponding variants in eCGS and eCBL (Lodha and Aitken, 2011; Jaworski et al., 2012). This suggests that in the context of yCGL, the hydroxyl group of this residue is essential for maintenance of active-site architecture and/or catalysis of L-Cth hydrolysis, supporting the hypothesized role of this residue. The differences in how these three structurally similar, but catalytically distinct enzymes employ this active-site tyrosine may reflect the more facile nature of the CBL-catalyzed $\beta$-elimination, which only requires proton abstraction from $C_{\alpha}$ (Clausen et al., 1996), while the $\gamma$-elimination of 
eCGS and yCGL involves proton transfer between $\mathrm{C}_{\alpha}$ and $\mathrm{C}_{\beta}$ of the substrate and C4' of the cofactor (Figure 1.9; Brzovic et al., 1990). The electronegative Y103 hydroxyl group, positioned $\sim 3 \AA$ above $C 4$ ' of the cofactor (Figure 1.8), may interact with and guide the catalytic lysine to ensure that protonation of the proper atom of the cofactor occurs. The lack of alterations in kinetic parameters for hydrolysis of L-Cys and L-OAS upon substitution of yCGL-Y103 with phenylalanine provides further confirmation that the step of catalysis which Y103 facilitates is specific to $\gamma$-elimination. This is exemplified by the 45 -fold greater $k_{\text {cat }}{ }^{\mathrm{L}-\mathrm{Cys}}$ compared to the $k_{\text {cat }}^{\mathrm{L}-\mathrm{Cth}}$, of the physiological L-Cth substrate, of the Y103F variant and strongly supports a role for Y103F in guiding K203 to $\mathrm{C}^{\prime}$ ', since protonation of $\mathrm{C}^{\prime}$ ' is not required in the $\beta$-elimination mechanism. This is agreement with the findings of Inoue et al. (2000) for the corresponding Y114F variant of Pseudomonas putida methionine $\gamma$-lyase (MGL), which displayed a 910 -fold-reduced $k_{\text {cat }}$ for the $\gamma$-elimination of L-methionine compared to the wild type, while the $k_{\text {cat }}$ for the hydrolysis of substrates such as $S$-methyl-L-cysteine requiring $\beta$-elimination was reduced by only 13 -fold, indicating that this residue plays a necessary role in $\gamma$-elimination mechanisms of both yCGL and MGL.

To investigate the possibility of the involvement of yCGL-Y103 in proton transfer, as proposed by Messerschmidt et al. (2003), the $\mathrm{pH}$ dependence of the specific activity and catalytic efficiency of the Y103F variant was investigated. The specific activity versus $\mathrm{pH}$ profile of the $\mathrm{Y} 103 \mathrm{~F}$ variant (Figure 3.11B) displays a $\mathrm{pH}$ optimum of $8.4-9$ and a basic limb $\mathrm{p} K_{\mathrm{a}}$ value of $9.9 \pm 0.1$, which represent increases in both cases of $\sim 1.1 \mathrm{pH}$ units from the wild type (Table 3.3). However, the $k_{\mathrm{cat}} / K_{\mathrm{m}}$ versus $\mathrm{pH}$ profile of Y103F showed little variation from that of the wild type (Figure 3.12), similar to the 
$k_{\text {cat }} / K_{\mathrm{m}}^{\mathrm{L}-\mathrm{Cth}}$ versus $\mathrm{pH}$ profile of eCBL-Y111F (Lodha and Aitken, 2011). The observation that substitution of this conserved tyrosine in either yCGL or eCBL with phenylalanine does not eliminate one of the limbs of the $k_{\mathrm{cat}} / K_{\mathrm{m}}^{\mathrm{L}-\mathrm{Cth}}$ versus $\mathrm{pH}$ profile indicates that this residue is not one of the titrating groups responsible for either of the $\mathrm{p} K_{\mathrm{a}}$ values of the $\mathrm{pH}$ dependence of $k_{\text {cat }} / K_{\mathrm{m}}^{\mathrm{L}-\mathrm{Cth}}$, and suggests that this conserved tyrosine does not directly participate in proton transfer in either enzyme. From the broader fold type I of PLPdependent enzymes, the corresponding Y121F variant in murine erythroid ALAS has also been shown to have similar $\mathrm{pH}$ dependence to the wild type (Tan et al., 1998). The conservation of an aromatic residue in this position across the large and catalytically diverse fold type I family of PLP-dependent enzymes suggests a common role for this residue (Hayashi, 1995; Messerschmidt et al., 2003). This is likely the $\pi$-stacking interactions with the pyridine ring of the cofactor, but this does not discount the potential for Y103 to have an essential role in yCGL in guiding the catalytic lysine to $\mathrm{C}^{\prime}$ ' to ensure protonation of the cofactor in a manner that leads to $\gamma$-elimination. The role of yCGLY103 in abstracting a proton from the $\alpha$-amino group of the substrate to initiate transaldimination, as proposed by Messerschmidt et al. (2003), can be ruled out given the near-wild-type $k_{\text {cat }}$ values for hydrolysis of L-Cys and L-OAS, both of which also require this step. Similarly, investigation of the corresponding MGL-Y114F variant has demonstrated that it is not involved in removal of protons (Inoue et al., 2000).

Evidence supporting a role for $\mathrm{Y} 103 \mathrm{~F}$ in modulating the reaction specificity of yCGL also lies in the unique changes observed in absorbance spectra of the PLP cofactor of Y103F (Figure 3.13). The lack of absorbance at $330 \mathrm{~nm}$ present after reaction of yCGL-Y103F with L-Cth (Figure 3.13B), lies in contrast to the 330-nm shoulder 
indicative of protonation of $\mathrm{C} 4$ ' due to formation of a ketimine derivative, as required for $\gamma$-elimination, observed following reaction of the wild-type enzyme with L-Cth (Figure $3.13 \mathrm{~B}$, inset). The lack of absorbance at $330 \mathrm{~nm}$ in the spectrum of $\mathrm{Y} 103 \mathrm{~F}$ after reaction with L-Cth suggests a decreased ability in Y103F to enforce protonation of the C4' atom of PLP (Steegborn et al., 1999), supporting a role for this residue in determining the reaction specificity of yCGL by guiding the catalytic lysine (K203) to C4' of PLP. While substitution of residues Y49 and S334, both of which have been proposed to tether/guide $\mathrm{K} 203$, results in similar effects of a decreased shift in absorbance from $420 \mathrm{~nm}$ to $330 \mathrm{~nm}$ after reaction with L-Cth, compared to the wild type (Figure 3.13B), substitution of Y103 with phenylalanine results in a complete absence of absorbance increase at $330 \mathrm{~nm}$ compared to the wild type and all other site-directed variants examined. The decrease in absorbance of the 420-nm band without concomitant increase in absorbance suggestive of formation of a characteristic intermediate is similar to the absorbance spectrum of the corresponding Y114F variant of MGL after reaction with L-methionine, which displays some quenching of the $420-\mathrm{nm}$ band, but little changes in the $300-350 \mathrm{~nm}$ region (Inoue et al., 2000). These spectral changes indicate that while Y49, S334, and Y103 of yCGL are all required to act in concert to enable catalysis in yCGL, residue Y103 plays the major role in ensuring protonation of $\mathrm{C}^{\prime}$. Incubation of $\mathrm{Y} 103 \mathrm{~F}$ with the alternative substrate L-alanine results in the appearance of a $330-340-\mathrm{nm}$ shoulder suggestive of PMP formation (Figure 3.13A), similar to the $320 \mathrm{~nm}$ shoulder observed for the corresponding eCGS-Y101F variant with L-alanine (Jaworski et al., 2012). This distinguishes yCGL-Y103F from the corresponding eCBL-Y111F, for which no suggestion of transamination activity has been reported (Lodha and Aitken, 2011). These 
observations indicate that the hydroxyl moiety of this tyrosine residue acts as a determinant of reaction specificity in eCGS and yCGL but not eCBL.

Replacement of the corresponding Y114 with phenylalanine, in the context of the human CGL enzyme, results in a 3.6-fold increase in L-Cys hydrolysis leading to the production of $\mathrm{H}_{2} \mathrm{~S}$ (Huang et al., 2010). While a corresponding increase in L-Cys hydrolysis for Y103F relative to the wildtype is not observed for the yCGL enzyme, Y103F carries out the $\beta$-elimination of L-Cys with a 45 -fold higher $k_{\text {cat }}$ than that for the Y103F-catalyzed L-Cth hydrolysis, which suggests that the hydroxyl group of the residue at this position acts as a determinant of $\gamma$-elimination specificity, perhaps by diminishing the ability of the enzyme to catalyze $\beta$-elimination. Preclusion of interaction between the Y103 hydroxyl group and the K203 $\varepsilon$-amino group, by the Y103F substitution, may hinder access or guidance of the catalytic lysine to position C4' of the cofactor, as protonation of this position is required for the $\gamma$ but not $\beta$-elimination reaction. Huang et al. (2010) suggested that the increased ability of hCGL-Y114F to produce $\mathrm{H}_{2} \mathrm{~S}$ arises from altered cofactor positioning due to weakening of $\pi$-stacking interactions from loss of the electronegative hydroxyl moiety of Y114. However, if this were the case, similar effects on the $\beta$ and $\gamma$-elimination reactions of the corresponding yCGL-Y103F would be expected, which is not observed (Table 3.6). Therefore, while altered cofactor positioning may affect the reaction specificity of yCGL, it is likely the role of this residue in guiding K203 to C4' of PLP that exerts a greater effect on $\gamma$-elimination reaction specificity.

\subsection{The $S 334 A$ variant.}


Residue S334, conserved in other fold type I enzymes of the transsulfuration pathways as eCGS-S326 and eCBL-S339 (Messerschmidt et al., 2003), has been demonstrated to be a determinant of reaction specificity in these enzymes by tethering and guiding the catalytic lysine to promote or prevent proton transfer between $\mathrm{C} 4$ ' of the cofactor and $\mathrm{C}_{\beta}$ of the substrate (Lodha and Aitken, 2011; Jaworski et al., 2012). The reduction in catalytic efficiency of the yCGL-S334A variant is dominated by a 630 -fold decrease in $k_{\text {cat }}$ for the hydrolysis of L-Cth (Table 3.6). This reduction is greater than the 40- and 360-fold decreases in this kinetic parameter obtained for the $\gamma$-elimination and $\gamma$ replacement activity, respectively, of the corresponding eCGS-S326A variant (Jaworski et al., 2012). However, this substitution has a more drastic effect in eCBL, as the $k_{\text {cat }}$ of LCth hydrolysis is decreased 5600-fold by the corresponding S339A substitution (Lodha and Aitken, 2011).

The undetectable activity of the S334A variant for hydrolysis of L-Cys, L-Hcys, and L-OAS, as well as the 630 -fold reduced $k_{\text {cat }}{ }^{\text {LCth }}$ (Table 3.6), imply that this residue plays an essential role in facilitating a catalytic step common to the hydrolysis of substrates requiring both $\beta$ - and $\gamma$-elimination, such as $\alpha$-proton abstraction. However, the specific activity versus $\mathrm{pH}$ profile of the S334A variant (Figure 3.11C) shows little variation from the wild type, indicating that, similar to Y49 and Y103, residue S334 facilitates catalysis and affects reaction specificity by tethering and restraining the catalytic lysine, but not through direct proton transfer.

Replacement of yCGL-S334 with alanine results in a 330-nm peak characteristic of PMP formation, and not observed for the wild-type enzyme, in the absorbance spectrum of the cofactor following reaction with the L-alanine substrate (Figure 3.13A). 
The appearance of this $330-\mathrm{nm}$ peak for the S334A suggests the occurrence of a minor transamination reaction (Toney and Kirsch, 1991; Cellini et al., 2007), indicating a loss of reaction specificity upon removal of the hydrogen-bonding ability of S334 by replacement with alanine. This is similar to the transamination activity observed for the corresponding eCBL-S339A variant (Lodha and Aitken, 2011), and is in keeping with a role for this conserved serine residue in modulating reaction specificity, in concert with Y49 and Y103F. However, this contrasts with the eCGS-S326A variant, which exhibits spectral characteristics identical to the wild-type eCGS enzyme (Jaworski et al., 2012), and illustrates the varied roles which conserved residues can play, even among structurally similar enzymes. Thus enzymatic reaction specificity is enforced not only by making the active-site conditions most favourable for the desired reaction, but by preventing other possible side reactions from occurring after formation of the external aldimine complex (Vacca et al., 1997).

The ability of S334 to act as a determinant of reaction specificity lies in its proximity to the catalytic lysine (Figure 1.8 ). These residues are separated by $\sim 5 \AA$ in the crystal structure of yCGL where K203 is in Schiff base linkage with the cofactor, but release of the flexible K203 side chain, upon binding of the substrate to the cofactor, would allow the $\varepsilon$-amino group of the catalytic base to approach the hydroxyl group of S334, enabling a hydrogen-bonding interaction to restrain and/or guide movement of the catalytic lysine. Loss of this tethering interaction is indicated by the 630 -fold decrease in $k_{\text {cat }}{ }^{\text {LCth }}$ upon replacement of yCGL-S334 with alanine (Table 3.6). The shorter distance of $3.5 \AA$ which separates the hydroxyl group of this conserved serine and the $\varepsilon$-amino group of the catalytic lysine in eCBL (Figure 1.8), as well as the more drastic, $\sim 5600$-fold 
reduction in $k_{\text {cat }}$ of eCBL-S339A, likely reflects the greater extent to which the catalytic lysine must be restrained in eCBL compared to yCGL, as protonation of $\mathrm{C4}$ ' of the cofactor must be prevented during a $\beta$-elimination but is required for $\gamma$-elimination (Brzovic et al., 1990; Clausen et al., 1996).

\subsection{The $E 48 A / D / Q$ variants.}

The residue at this position was proposed to bind the distal amino group of L-Cth in eCBL and yCGL and the corresponding $\alpha$-amino group of L-Cys in eCGS (Clausen et al., 1996; Clausen et al., 1998; Messerschmidt et al., 2003). Residue E48 of yCGL is conserved as an acidic residue (D45) in eCGS but is replaced by an aromatic residue (F55) in eCBL (Figure 1.7). Therefore, this residue, in combination with yCGL-E333, was proposed to act as a determinant of reaction specificity (Clausen et al., 1996; Clausen et al., 1998; Messerschmidt et al., 2003). However, the near-wild type kinetic parameters for hydrolysis of L-Cth, L-Cys, and L-OAS by the E48A/D/Q variants (Table 3.7) do not support the hypothesized role of this residue, and suggest that the residue at this position does not play an essential role in the binding or catalysis of these substrates in yCGL. Additionally, the spectral changes of the PLP cofactor of the E48A/D/Q variants after reaction with L-alanine and L-Cth are similar to the wild type (Figure 3.15), indicating that replacement of E48 is not sufficient to alter the distribution of reactive intermediates formed during catalysis and that E48 is not a direct determinant of substrate or reaction specificity in the context of the yCGL active-site. 
Interestingly, the $k_{\mathrm{cat}} / K_{\mathrm{m}}^{\mathrm{L}-\mathrm{Cth}}$ versus $\mathrm{pH}$ profiles of the E48A and E48Q substitution variants are both shifted in their $\mathrm{pH}$ optima to $7.8-8.2$ (Figure 3.14). This represents an increase of approximately $0.6 \mathrm{pH}$ units, compared to the wild-type optimum of $7.2-7.8$. In contrast, the $\mathrm{pH}$ dependence of the E48D variant, which maintains the negatively charged carboxylate moiety of the side chain, is similar to the wild type enzyme (Figure 3.14). The shift in $\mathrm{pH}$ profiles of the $\mathrm{E} 48 \mathrm{~A} / \mathrm{Q}$ variants is surprising, given the near-wild-type kinetic parameters of these variants for the hydrolysis of L-Cth, L-Cys, and L-OAS. This observation demonstrates that while it may not be directly involved in substrate binding or catalysis or act as a primary determinant of specificity, the deprotonated carboxylate group of yCGL-E48 may play a role in maintaining optimal active-site charge distribution. The negligible alterations in steady-state kinetic parameters between E48 variants and the wild type compared to their differences in $\mathrm{pH}$ dependence highlight the multitude of subtle interactions upon which enzymatic catalysis is dependent, and illustrate the importance of additional characterizations of site-directed variants in determining the roles and contributions of active-site residues to this process.

Replacement of the corresponding D45 of eCGS with alanine or asparagine results in only 2-9-fold changes in kinetic parameters but enables a minor transamination activity (Jaworski et al., 2012), showing that while this residue is positioned close to the L-Cys-binding site, it does not participate directly in substrate binding in eCGS but may affect active-site dynamics to subtly influence reaction specificity. The near-wild-type kinetic parameters of the $y C G L E 48 \mathrm{~A} / \mathrm{D} / \mathrm{Q}$ variants are similar to what is observed in eCGS; however, the spectral characteristics of the $y C G L-E 48 \mathrm{~A} / \mathrm{D} / \mathrm{Q}$ variants were unchanged from the wild type. 
Substitution of the corresponding F55 of eCBL with the aspartate found at the same position in eCGS results in a 78 -fold increase in $K_{\mathrm{m}}{ }^{\mathrm{L} \text { Cth }}$ (Farsi et al., 2009), suggesting that the identity of the residue at this position is a partial determinant of substrate specificity. These results suggest that an aromatic residue at this position may serve to orient the L-Cth substrate properly for $\beta$-elimination in $\mathrm{eCBL}$, while in eCGS and $\mathrm{yCGL}$, the presence of an acidic residue at this position plays a more subtle, indirect role in affecting the specificity of the enzyme through substrate positioning or charge distribution within the active-site.

\subsection{The $E 333 A / D / Q$ variants.}

Residue E333 of yCGL was proposed to interact with the distal amino group of LCth (Figure 1.5), in addition to as acting as a determinant of reaction specificity, via electrostatic repulsion between its carboxylate side chain and the sulfur atom of L-Cth, thereby favouring binding in the conformation that would lead to a $\gamma$-elimination reaction (Messerschmidt et al., 2003). A substrate-binding role is confirmed for this residue in the context of the yCGL active site, supporting the hypothesized role of this residue, as replacement with alanine, aspartate or glutamine causes a $16-18$-fold increase in $K_{\mathrm{m}}{ }^{\text {L-Cth }}$ (Table 3.8). However, the kinetic parameters for L-OAS hydrolysis by the E333A/D/Q variants are within 2.5 -fold of the wild type enzyme (Table 3.8). The lack of an increase in $K_{\mathrm{m}}{ }^{\mathrm{LOAS}}$, such as that observed for $K_{\mathrm{m}}{ }^{\mathrm{L}-\mathrm{Cth}}$, suggests that $\mathrm{E} 333$ interacts with the distal amino group of L-Cth (Figure 1.5; Messerschmidt et al., 2003), as this group is not present in L-OAS. Therefore, the results of this study demonstrate that the distal 
carboxylate and amino moieties of L-Cth are bound by residues R51, R108 and N232 and by E333, respectively, of yCGL.

In the human CGL enzyme, substitution of the corresponding glutamate residue (hCGL-E339) with lysine, alanine, and tyrosine increased the rate of $\mathrm{H}_{2} \mathrm{~S}$ production from the $\beta$-elimination of L-Cys by $\sim 2,3$, and 7 -fold respectively (Huang et al., 2010), suggesting that the hydrophobicity of this residue, not its identity, affects the $\beta$ elimination activity of this enzyme. Greater hydrophobicity of the residue at this position was proposed to decrease potential electrostatic repulsion interactions with the substrate, thereby allowing it to adopt a conformation optimal for $\beta$-elimination (Messerschmidt et al., 2003). The 3-fold increase in catalytic efficiency of $\mathrm{H}_{2} \mathrm{~S}$ production from L-Cys by the E339A variant of hCGL, reported by Huang et al. (2010), appeared to support this hypothesis; however, a similar increase in $\beta$-elimination activity was not observed for yCGL-E333A, as the $k_{\text {cat }} / K_{\mathrm{m}}{ }^{\mathrm{L}-\mathrm{Cys}}$ of this variant is 2 -fold lower than the wild-type enzyme (Table 3.8). Additionally, the 2009 study by Farsi and collegues demonstrated that the yCGL-E333Y mutation was not sufficient to alter the in vivo reaction specificity of yCGL. However, a role for this residue in modulating the reaction specificity of yCGL, possibly via orientation of the substrate through binding the distal amino group, is supported by $K_{\mathrm{m}}{ }^{\mathrm{L}-\mathrm{Cth}}$ increases and the altered spectral characteristics after incubation of the yCGL-E333A/D/Q variants with the alternate-reaction substrate alanine. The differences in kinetic parameters of site-directed replacement variants of this residue between the yeast and human CGL enzymes may be due to different kinetic assays employed in this study, which employed a continuous assay to detect the pyruvate product, compared to those of Huang and colleagues, who employed an endpoint assay to 
detect the $\mathrm{H}_{2} \mathrm{~S}$ product, of L-Cys hydrolysis. Therefore, the observed modest relative changes may not be representative of underlying biochemical differences in these enzymes, but may reflect differences in experimental conditions. Another source of the discrepant results reported for the corresponding hCGL-E339A and yCGL-E333A variants could be the specificity of the assay. While the LDH assay employed in this study detects pyruvate arising from $\beta$-elimination, the assay employed by Huang and colleagues measures total $\mathrm{H}_{2} \mathrm{~S}$ produced, which can be formed through $\beta$-elimination (LCys $\rightarrow \mathrm{H}_{2} \mathrm{~S}+$ pyruvate $+\mathrm{NH}_{3}$ ) or $\beta$-replacement (2 L-Cys $\rightarrow \mathrm{H}_{2} \mathrm{~S}+$ lanthionine) (Figure 1.10B) reactions of the L-Cys substrate (Chiku et al., 2009). Therefore, the increase in $\mathrm{H}_{2} \mathrm{~S}$ production observed for hCGL-E339A may result from increased $\beta$-elimination or replacement activity. In order to fully examine roles of CGL residues in $\mathrm{H}_{2} \mathrm{~S}$ production, additional assay(s) are required to differentiate between elimination and replacement activities with alternative substrates.

Although the electrostatic repulsion proposal of Messerschmidt et al. (2003) is not supported, the spectral characteristics of the $\mathrm{E} 333 \mathrm{~A} / \mathrm{D} / \mathrm{Q}$ variants do support a role for this residue in influencing reaction specificity. Substitution of E333 does not alter the absorbance spectra of the yCGL-E333A/D/Q variants after reaction with L-Cth (Figure 3.17B). However, after reaction with L-alanine, a 330-nm peak appears in the absorbance spectra of the E333A/D/Q variants (Figure 3.17A), which is similar to the $320-\mathrm{nm}$ absorbance reported for the corresponding eCGS-E325A variant after reaction with alanine (Jaworski et al., 2012), but not observed for the wild-type yCGL enzyme (Figure 3.17A, inset). While the side-chain carboxylate of E333 is $\sim 11 \AA$ from the PLP phosphate in the yCGL crystal structure (Messerschmidt et al., 2003), a conformational change upon 
substrate binding may bring these two groups in closer proximity, allowing the deprotonated glutamate side chain to affect the charge environment around the PLP phosphate, affecting positioning of the cofactor through electrostatic repulsion between these groups. Alternatively, residue E333 may influence the position of the cofactor via interactions mediated by $\mathrm{R} 51$ and/or R108, a possibility supported by the proximity of these residues in the enzyme-substrate complex, given their demonstrated roles in binding to the distal portion of the L-Cth substrate. Removal or alteration of this putative interaction upon substitution of the E333 side chain may allow the substrate and cofactor to adopt different relative orientations, resulting in the loss of reaction specificity observed after incubation with $\mathrm{L}$-alanine. This indicates that both the length of side chain and presence of a carboxylate functional group at this position contribute to maintenance of reaction specificity in CGL.

The $\mathrm{p} K_{\mathrm{a}}$ of the acidic limb of the $k_{\text {cat }} / K_{\mathrm{m}}{ }^{\mathrm{L}-\mathrm{Cth}}$ versus $\mathrm{pH}$ profile of both the E333A and E333Q variants is increased by $0.6-0.9 \mathrm{pH}$ units (Table 3.4), and the $\mathrm{pH}$ optimum is shifted to $8.4-8.8$ and $7.8-8.6$, respectively, which represent increases of $0.6-1 \mathrm{pH}$ unit compared to the wild-type enzyme (Figure 3.16 ). In contrast, the pH optimum and $\mathrm{p} K_{\mathrm{a}}$ values of $k_{\mathrm{cat}} / K_{\mathrm{m}}{ }^{\mathrm{L}-\mathrm{Cth}}$ versus $\mathrm{pH}$ profile of the E333D variant (Figure 3.16; Table 3.4), which maintains the carboxylate moiety of the side chain at this position but decreases its length by $\sim 1 \AA$, are within experimental error of wild-type yCGL. The observation that the presence of a carboxylate group in the E333D variant is sufficient to maintain the wild-type $\mathrm{pH}$ profile indicates that a negatively-charged side chain at position 333 fulfils a required charge distribution in the active site. Additionally, the shift in $\mathrm{pH}$ dependence of the E333Q variant, compared to that of E333D and the wild type (Figure 3.16), 
indicates that the presence of a deprotonated carboxylate group is more important than maintaining the length of the side chain at this position for wild-type-like $\mathrm{pH}$ dependence. Given the interaction between the side chain of E333 and the distal amino group of L-Cth, a negatively charged group may be necessary at this position for optimal substrate positioning.

The eCBL enzyme possesses an aromatic residue (Y338) at the corresponding position of yCGL-E333 (Figure 1.7). Residue eCBL-Y338 does not interact with the LCth substrate, as the kinetic parameters of the phenylalanine replacement variant of this residue are within 2-fold of wild-type eCBL (Lodha and Aitken, 2011). Similar to yCGL, this residue in eCGS is conserved as a glutamate, but in contrast to the 16-18-fold increases in $K_{\mathrm{m}}{ }^{\text {LCth }}$ observed for yCGL-E333A/D/Q (Table 3.8), the corresponding variants of eCGS-E325, the physiological substrate of which is L-OSHS, which lacks the distal amino group of L-Cth, displayed 2 - 4-fold decreases in $K_{\mathrm{m}}{ }^{\mathrm{L}-\mathrm{OSHS}}$ (Jaworski et al., 2012). This comparison suggests that in eCGS the carboxylate portion of the E325 side chain may serve to deter substrates from binding in a non-productive orientation, while in yCGL the corresponding E333 forms a direct interaction with the distal amino group of the substrate (Messerschmidt et al., 2003). These distinct alterations in kinetic parameters of the $y C G L-E 333 \mathrm{~A} / \mathrm{D} / \mathrm{Q}$ variants, compared to the functions of the corresponding residues in $\mathrm{eCBL}$ and $\mathrm{eCGS}$, combined with the spectral changes characteristic of altered PLP protonation after reaction of the E333Q variant with L-alanine, point to a unique role for this residue in determining the reaction specificity of $y C G L$ through positioning of LCth via hydrogen bonding with the distal amino group of the substrate. 


\subsection{The E48/E333 alanine, aspartate, and glutamine double-replacement variants.}

The E48/E333 double replacement variants were constructed to investigate potential additive effects of these two residues proposed to modulate the reaction specificity of CGL. Double substitution of both E48 and E333 with alanine, aspartate, or glutamine resulted in greater perturbations of the kinetic parameters of yCGL for L-Cth hydrolysis than substitution of either E48 or E333 alone. The E48/E333 double replacement variants are not saturated within the solubility limit of the L-Cth substrate and the $k_{\text {cat }} / K_{\mathrm{m}}^{\mathrm{L}-\mathrm{Cth}}$ is reduced by up to 560 -fold, compared to the wild type (Table 3.9). This represents a more drastic reduction than the 2-fold and 30-60-fold decreases in catalytic efficiency observed for the single substitution variants of E48 (Table 3.7) and E333 (Table 3.8), respectively. This indicates that while E333 plays an important role in substrate binding, as evidenced by the $16-18$-fold increase in $K_{\mathrm{m}}^{\mathrm{L}-\mathrm{Cth}}$ observed for single substitutions at this position, the additive effect of replacement of both glutamate residues alters active-site architecture to a greater extent such that the ability of the L-Cth substrate to bind productively is diminished. Similar to the single replacement variants of residue E333, the near-wild-type kinetic parameters for L-OAS hydrolysis by the E48/E333 double replacement variants (Table 3.9) support a role for the E333 residue in binding the distal amino of $L-C$ th, as L-OAS lacks this group.

The $k_{\text {cat }} / K_{\mathrm{m}}{ }^{\text {L-Cth }}$ versus $\mathrm{pH}$ profiles of the E48/E333 alanine and glutamine double substitution variants are shifted, as indicated by the increase of $\sim 2 \mathrm{pH}$ units in the $\mathrm{pH}$ optimum to 9-10 and 8.8-9.8, respectively (Figure 3.18). The $\mathrm{p} K_{\mathrm{a}}$ values of the acidic limb of these double substitution variants are also increased, by $\sim 1 \mathrm{pH}$ unit compared to the wild type (Table 3.4). The increased magnitude of these shifts compared to the 
corresponding single substitutions of E48 (Figure 3.14) and E333 (Figure 3.16), which increased the $\mathrm{pH}$ optimum and acidic limb $\mathrm{p} K_{\mathrm{a}}$ values by $0.5-1 \mathrm{pH}$ unit (Table 3.4), indicates that these two residues work in concert to provide optimal charge distribution in the active site, particularly in the region of the binding site of the distal portion of the LCth substrate. While single substitution of E48 or E333 with aspartate does not alter the pH dependence of $k_{\text {cat }} / K_{\mathrm{m}}{ }^{\text {L-Cth }}$ (Figures 3.14 and 3.16), the pH optimum of the E48D/E333D double substitution variant is $\sim 1 \mathrm{pH}$ unit higher than the wild type (Figure 18B). This indicates that both the presence of a carboxylate group and the length of side chain at both positions affect the $\mathrm{pH}$ dependence of $\mathrm{yCGL}$, and provides further evidence that residues $\mathrm{E} 48$ and $\mathrm{E} 333$, separated by only $\sim 6 \AA$ in the yCGL crystal structure, may act synergistically to maintain optimal charge distribution in the active site around the distal portion of the L-Cth substrate.

The wild-type yCGL and eCBL enzymes have distinct $\mathrm{pH}$ profiles, with $\mathrm{pH}$ optima of 7.6-8 (Figure 3.4) and 8.5-9.5 (Lodha and Aitken, 2011), respectively. The increased $\mathrm{pH}$ optima of the double replacement variants create a $\mathrm{pH}$ profile similar to that of eCBL (Lodha and Aitken, 2011). Interestingly, residues E48/E333 of yCGL are replaced with aromatic residues F55 and Y338 in eCBL (Figure 1.7), suggesting that the presence of acidic residues at these positions may be partially responsible for the distinct $\mathrm{pH}$ profile of yCGL, possibly by affecting the active-site charge distribution to enable $\mathrm{L}$ Cth binding in a manner conducive for $\gamma$-elimination.

The alanine, aspartate, and glutamine double replacement variants of E48/E333 displayed similar spectral characteristics to wild-type yCGL after reaction with L-Cth (Figure 3.19B). However, appearance of the 330-nm band suggestive of PMP formation 
in the spectra of the $\mathrm{E} 48 / \mathrm{E} 333 \mathrm{~A} / \mathrm{D} / \mathrm{Q}$ double variants after incubation with L-alanine, which is not observed for the wild type (Figure 3.19A), is similar to those observed for the corresponding E333A/D/Q single-replacement variants (Figure 3.17) and indicates that residue E333 is a greater contributor than E48 to the altered reaction specificity observed for the E48/E333 double replacement variants with L-alanine.

The ability of E333, possibly in concert with E48, to influence reaction specificity may be a result of its proximity to the adjacent $S 334$, which tethers/guides the catalytic lysine. The negatively-charged environment provided by the nearby E48 and E333 may affect the tethering interaction between S334 and K203, making it more favourable for the $\varepsilon$-amino group of the catalytic base to approach S334. This provides a possible explanation of why a change in reaction specificity is observed for reaction with the smaller L-alanine substrate as the lack of a distal amino group to interact with E333 allows repositioning of this residue, thereby modifying its indirect effect on the positioning of $\mathrm{K} 203$. Therefore, the combined effect of orienting the substrate through hydrogen bonding with the distal amino group, with possibly influencing the tethering interaction between S334 and K203, may allow E333 to affect reaction specificity in yCGL. In contrast, the corresponding Y338 of eCBL forms a hydrogen bond to eCBL$\mathrm{K} 42$, situated in a segment of eight amino acids not present in eCGS or yCGL (Lodha and Aitken, 2011), which may tether this side chain with respect to eCBL-S339 and the active-site lysine and prevent eCBL-Y338 from having a similar role to yCGL-E333.

The identity of the residue at the nearby 335 position may also influence the positioning/orientation of residues E333 and S334, positioning them to result in the desired orientation for a $\beta$ - or $\gamma$-elimination reaction. This position is conserved as a 
hydrophobic residue in the $\gamma$-subfamily of fold type I. A leucine occupies this position in yCGL, hCGL, MGL and eCGS, but in CBL from E. coli and Arabidopsis thaliana it is replaced by a tryptophan or phenylalanine residue, respectively (Messerschmidt et al., 2003). Residue eCBL-W340 is conserved in bacterial CBL enzymes and forms hydrogen bonds to the $\mathrm{O}^{\prime}$ ' position of the PLP cofactor and the $\alpha$-carboxylate group of the substrate, thereby providing a tethering interaction distinct from yCGL-E333 (Lodha and Aitken, 2011). The absence of the bulky aromatic side chain at the position corresponding to yCGL-L335 (eCBL-W340) in enzymes catalyzing $\gamma$-eliminations may allow residues E333/S334 to adopt distinct orientations, resulting in the greater importance of E333 in affecting reaction specificity of yCGL compared to eCBL. 


\section{CONCLUSION}

The goal of this research was to characterize the roles of active-site residues in determining the substrate and reaction specificity of yCGL, by testing the following hypotheses:

1. residues $\mathrm{R} 51, \mathrm{~S} 52, \mathrm{R} 108, \mathrm{~N} 232$ and $\mathrm{R} 369$ interact with the L-Cth substrate;

2. residue $\mathrm{Y} 49$ participates in substrate binding, and $\mathrm{Y} 103$ and $\mathrm{S} 334$ facilitate catalysis and are determinants of specificity in yCGL;

3. residues E48 and E333 interact with L-Cth and are determinants of substrate specificity in yCGL.

This study has confirmed roles for residues R51, R108, and N232 in binding the distal carboxylate of the L-Cth substrate in yCGL, as hypothesized, and revealed potential roles for these residues in influencing enzyme specificity by maintaining the proper orientation of the substrate. The hydrogen bonds formed between the guanidinium group of R51 and the PLP phosphate is an interaction conserved among the fold type I transsulfuration enzymes, and tethering of the cofactor in this manner appears to affect reaction specificity by positioning the substrate-PLP complex in the most productive conformation. Residue N232 is replaced with a tyrosine in the eCBL enzyme, and both residues at this position interact with the distal carboxylate of L-Cth. However, the increased hydrogen-bonding capacity of asparagine compared to tyrosine allows this residue to play an additional role in yCGL in positioning the PLP cofactor, via interaction with R51, to enforce reaction specificity. Thus the identity of the amino acid at this position is a partial determinant of substrate and reaction specificity in yCGL. Residue 
$\mathrm{S} 52$, in contrast to its hypothesized role, does not play a major role in substrate-binding and is not a determinant of substrate or reaction specificity in yCGL. Supporting its hypothesized role, residue R369 binds the $\alpha$-carboxylate of the L-Cth substrate, and this role is conserved in the corresponding residues of eCGS and $\mathrm{CCBL}$.

Residue Y49 is also a partial determinant of reaction specificity, a function conserved in eCGS and eCBL, a role likely mediated by the hydrogen bond formed between the hydroxyl moiety of this side chain and the PLP phosphate. While Y49 does not appear to interact with the substrate as hypothesized, within the yCGL active site Y49 appears to play an additional role in facilitating catalysis, which is not observed for the corresponding residues of eCGS and eCBL. In contrast with its predicted role, Y103 is not involved in proton transfer, but does facilitate a step of catalysis specific to $\gamma$ elimination, likely by guiding the catalytic base, residue $\mathrm{K} 203$, to $\mathrm{C} 4$ ' of the cofactor for protonation. This function of Y103 is similar to that of the corresponding tyrosine in hCGL and MGL, but distinct from the corresponding residues of eCGS and eCBL, demonstrating the unique role of this residue as a determinant of $\gamma$-elimination specificity. The tethering interaction of S334 with the catalytic lysine is essential for catalysis and is a partial determinant of reaction specificity, similar to the function of this conserved residue in eCGS and eCBL.

Residue E48 does not form a direct interaction with the substrate, in contrast to its hypothesized function. A role for residue E333 in binding the distal amino group of L-Cth is supported, which is unique to yCGL and may contribute to the reaction specificity of this enzyme, as the corresponding eCBL-Y338 and eCGS-E325 do not interact with the substrate. Double replacement variants of E48 and E333 have demonstrated that these 
residues act in concert to maintain optimal charge distribution within the yCGL active site. This study forms the basis of an understanding of the CGL active site required for the design of antibiotic/herbicidal compounds to selectively inhibit the bacterial/plant transsulfuration enzymes by identifying unique active-site binding interactions, such as that of E333. This study also provides a foundation for enzyme engineering studies with the goal of engineering PLP-dependent enzymes to modify substrate or reaction specificity. For example, as important determinants of reaction specificity, residues Y103 and E333 would be potential targets of studies aiming to modify reaction specificity, while residues R51, R108 and N232 may be substituted, alone or in combination, to alter substrate specificity. This study characterized the contributions of active-site residues to the $\beta$ - versus $\gamma$-elimination reaction specificity of yCGL, forming the basis of the understanding that will be required for the design of antihypertensive treatments targeting $\mathrm{H}_{2} \mathrm{~S}$ production. For example, the role of $\mathrm{Y} 49$ in facilitating a catalytic step common to $\beta$ and $\gamma$-elimination identifies this residue as essential for the liberation of $\mathrm{H}_{2} \mathrm{~S}$ from Lcysteine by $\mathrm{CGL}$, the main enzyme responsible for $\mathrm{H}_{2} \mathrm{~S}$ production in the vasculature. However, since $\mathrm{H}_{2} \mathrm{~S}$ may arise from both elimination and replacement reactions, further investigation with assays to differentiate between the elimination/replacement products of the activity of yCGL site-directed variants will additionally enhance the understanding of the roles of key active-site residues in $\mathrm{H}_{2} \mathrm{~S}$ production. 


\section{REFERENCES}

Abe K. and Kimura H. 1996. The possible role of hydrogen sulphide as an endogenous neuromodulator. The Journal of Neuroscience 16: 1066-1071.

Aitken S.M. and Kirsch J.F. 2003. Kinetics of the yeast cystathionine $\beta$-synthase forward and reverse reactions: continuous assays and the equilibrium constant for the reaction. Biochemistry 42: 571-578.

Aitken S.M., Kim D.H. and Kirsch J.F. 2003. Escherichia coli cystathionine $\gamma$-synthase does not obey ping-pong kinetics: novel continuous assays for the elimination and substitution reactions. Biochemistry 42: 11297-11306.

Aitken S.M., Lodha P.H. and Morneau D.J.K. 2011. The enzymes of the transsulfuration pathways: Active-site characterizations. Biochimica et Biophysica Acta 1814: 1511-1517.

Atmaca G. 2004. Antioxidant effects of sulfur-containing amino acids. Yonsei Medical Journal 45: 776-788.

Bach R.D., Canepa C., and Glukhovtsev M.N. 1999. Influence of electrostatic effects on activation barriers in enzymatic reactions: pyridoxal 5-phosphate-dependent decarboxylation of $\alpha$-amino acids. J. Am. Chem. Soc. 121: 6542-6555.

Brosnan J.T. and Brosnan M.E. 2006. The sulfur-containing amino acids: an overview. The Journal of Nutrition 136: 1636S-1604S.

Brzovic P., Holbrook E.L., Greene R.C., and Dunn M.F. 1990. Reaction mechanism of Escherichia coli cystathionine $\gamma$-synthase: direct evidence for a pyridoxamine derivative of vinylglyoxylate as a key intermediate in pyridoxal phosphate dependent $\gamma$-elimination and $\gamma$-replacement reactions. Biochemistry 29: $442-451$.

Cellini B., Bertoldi M., Montioli R., Paiardini A., and Borri Voltattorni C. 2007. Human wildtype alanine:glyoxylate aminotransferase and its naturally occurring G82E variant: functional properties and physiological implications. Biochem J. 408: 39-50.

Chen X., Jhee K.H., and Kruger W.D. 2004. Production of the neuromodulator $\mathrm{H}_{2} \mathrm{~S}$ by cystathionine $\beta$-synthase via the condensation of cysteine and homocysteine. The Journal of Biological Chemistry 279: 52082-52086.

Cherest H., Thomas, D. and Surdin-Kerjan Y. 1993. Cysteine biosynthesis in Saccharomyces cerevisiae occurs through the transsulfuration pathway which has been built up by enzyme recruitment. Journal of Bacteriology 175: 5366-5374.

Chiku T., Padovani D., Zhu W., Singh S., Vivitsky V., and Banerjee R. 2009. H2S biogenesis by human cystathionine $\gamma$-lyase leads to the novel sulphur metabolites 
lanthionine and homolanthionine and is responsive to the grade of hyperhomocysteinemia. The Journal of Biological Chemistry 284: 11601-11612.

Christen P. and Mehta P.K. 2001. From cofactor to enzymes: the molecular evolution of pyridoxal-5'-phosphate-dependent enzymes. The Chemical Record 1: 436-447.

Clausen T., Huber R., Laber B., Pohlenz H.D., and Messerschmidt A. 1996. Crystal structure of the pyridoxal-5'-phosphate dependent cystathionine $\beta$-lyase from Escherichia

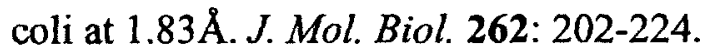

Clausen T., Huber R., Messerschmidt A., Pohlenz H.D., and Laber B. 1997. Slowbinding inhibition of the pyridoxal-5'-phosphate dependent cystathionine $\beta$-lyase by Laminoethoxyvinylglycine: a kinetic and X-ray study. Biochemistry 36: 12633-12643.

Clausen T., Huber R., Prade L., Wahl M.C., and Messerschmidt M. 1998. Crystal structure of Escherichia coli cystathionine $\gamma$-synthase at $1.5 \AA$ resolution. The EMBO Journal 17: 6827-6838.

Cook P.F., Hara S., Nalabolu S., and Schnackerz K.D. 1992. pH dependence of the absorbance and 31P NMR spectra of O-acetylserine sulfhydrylase in the absence and presence of O-acetyl-L-serine. Biochemistry 31: 2298-2303.

Cooper, A.J.L. 1983. Biochemistry of Sulfur-Containing Amino Acids. Ann. Rev. Biochem. 52: 187-222.

Datko A.H., Giovanelli J., and Mudd S.H. 1974. Homocysteine biosynthesis in green plants. J. Biol. Chem. 249: 1139-1155.

Diwakar L. and Ravindranath V. 2007. Inihibition of cystathionine- $\gamma$-lyase leads to loss of glutathione and aggravation of mitochondrial dysfunction mediated by excitatory amino acid in the CNS. Neurochemistry International 50: 418-426.

Dominy J.E. and Stipanuk M.H. 2004. New roles for cysteine and transsulfuration enzymes: production of $\mathrm{H}_{2} \mathrm{~S}$, a neuromodulator and smooth muscle relaxant. Nutrition Reviews 62: 348-353.

Dunathan H.C. 1966. Conformation and reaction specificity in pyridoxal phosphate enzymes. Proc. N.A.S. 55: 712-716.

Ellman G.L. 1959. Tissue sulfhydryl groups. Arch. Biochem. Biophys. 82: 70-77.

Eliot A.C. and Kirsch J.F. 2002. Modulation of internal aldimine pKa's of 1aminocyclopropane-1-carboxylate synthase and aspartate aminotransferase by specific active site residues. Biochemistry 41: 3836-3842. 
Eto K., Asada T., Arima K., Makifuchi T., Kimura H. 2002. Brain hydrogen sulphide is severely decreased in Alkheimer's disease. Biochemical and Biophysical Research Communications 293: 1485-1488.

Farsi A., Lodha P.H., Skanes J.E., Los H., Kalidindi N, and Aitken S.M. 2009. Interconversion of a pair of active-site residues in Escherichia coli cystathionine $\gamma$ synthase, $E$. coli cystathionine $\beta$-lyase, and Saccharomyces cereisiae cystathionine $\gamma$ lyase and development of tools for the investigation of their mechanisms and reaction specificity. Biochem. Cell. Biol. 87: 445-457.

Finkelstein J.D. 1998. The metabolism of homocysteine: pathways and regulation. Eur. J. Pediatr. 157: S40-S44.

Finkelstein J.D. and Martin J.M. 2000. Homocysteine. The International Journal of Biochemistry and Cell Biology 32: 385-389.

Goldberg J.M., Swanson R.V., Goodman H.S., and Kirsch J.F. 1991. The tyrosine-225 to phenylalanine mutation of Escherichia coli aspartate aminotransferase results in an alkaline transition in the spectrophotometric and kinetic $\mathrm{p} K_{\mathrm{a}}$ values and reduced values of both $k_{\text {cat }}$ and $K_{\mathrm{m}}$. Biochemistry 30: 305-312.

Hayashi H. 1995. Pyridoxal enzymes: mechanistic diversity and uniformity. J. Biochem. 118: $463-473$.

Higuchi R., Krummel B., and Saiki R.K. 1988. A general method of in vitro preparation and specific mutagenesis of DNA fragments. Nucleic Acids Research 16: 7351-7367.

Huang S., Chua J.H., Yew W.S., Sivaraman J., Moore P.K., Tan C.H., Deng L.W. 2010. Site-directed mutagenesis on human cystathionine- $\gamma$-lyase reveals insights into the modulation of $\mathrm{H}_{2} \mathrm{~S}$ production. J. Mol. Biol. 396: 708-718.

Inoue H., Inagaki K., Adachi N., Tamura T., Esaki N., Soda K., and Tanaka H. 2000. Role of tyrosine 114 of L-methionine $\gamma$-lyase from Pseudomonas putida. Biosci. Biotechnol. Biochem. 64: 2336-2343.

Jansonius J.N. 1998. Structure, evolution and action of vitamin B6-dependent enzymes. Current Opinion in Structural Biology 8: 759-769.

Jaworski A.F., Lodha P.H., Manders A.L., and Aitken S.M. 2012. Exploration of the active-site of Escherichia coli cystathionine $\gamma$-synthase. Protein Science 21: 1662-1671.

Jhee K.H., McPhie P., and Miles, E.W. 2000. Domain architecture of the hemeindependent yeast cystathionine $\beta$-synthase provides insights into mechanisms of catalysis and regulation. Biochemistry 39: 10548-10556. 
Kashiwamata S. and Greenberg D.M. 1970. Studies on cystathionine synthase of rat liver: properties of the highly purified enzyme. Biochimica et Biophysica Acta 212: 488 - 500 .

Kery V., Bukovska G., and Kraus J.P. 1994. Transsulfuration depends on heme in addition to pyridoxal 5'-phosphate: cystathionine $\beta$-synthase is a heme protein. The Journal of Biological Chemistry 269: 25283-25288.

Kirsch J.F., Eichele G., Ford G.C., Vincent M.G., and Jansonius J.N. 1984. Mechanism of action of aspartate aminotransferase on the basis of its spatial structure. J. Mol Biol. 174: $497-525$.

Kraus J.P., Hasek J., Kozic V., Collard R., Venezia S., Janosikova B., et al. 2009. Cystathionine $\gamma$-lyase: clinical, metabolic, genetic, and structural studies. Molecular Genetics and Metabolism 97: 250-259.

Lodha P.H., Jaworski A.F., and Aitken S.M. 2010. Characterization of site-directed mutants of residues R58, R59, D116, W340 and R372 in the active site of E. coli cystathionine beta-lyase. Protein Science 19: 383-391.

Lodha P.H. and Aitken S.M. 2011. Characterization of the side-chain hydroxyl moieties of residues Y56, Y111, Y238, Y338 and S339 as determinants of specificity in E. coli cystathionine $\beta$-lyase. Biochemistry 50: 9876-85.

Marangoni A.G. 2003. Enzyme kinetics: a modern approach. John Wiley and Sons, Hoboken, NJ. pp.79-89.

Messerschmidt A., Worbs M., Steegborn C., Wahl M.C., Huber R., Laber B., and Clausen T. 2003. Determinants of enzymatic specificity in the Cys-Met-metabolism PLPdependent enzymes family: crystal structure of cystathionine $\gamma$-lyase from yeast and intrafamiliar structure comparison. Biol. Chem. 384: 373-386.

Morneau D.J.K., Abouassaf E., Skanes J.E., and Aitken S.M. 2012. Development of a continuous assay and steady-state characterization of Escherichia coli threonine synthase. Analytical Biochemistry 423: 78-85.

Ono B.I., Hazu T., Yoshida S., Kawato T., Shinoda S., Brzvwczy J., and Paszewski A. 1999. Cysteine biosynthesis in Saccharomyces cerevisiae: a new outlook on pathway and regulation. Yeast 15: 1365-1375.

Pazicni S., Lukat-Rodgers G.S., Oliveriusova J., Rees K.A., Parks R.B., Clark R.W. Rodgers K.R., Kraus J.P. and Burstyn J.N. 2004. The redox behaviour of the heme in cystathionine $\beta$-synthase is sensitive to $\mathrm{pH}$. Biochemistry 43: 14684-14695. 
Peracchi, A., Bettati, S., Mozzarelli, A., Rossi, G. L., Miles, E. W., and Dunn, M. F. 1996. Allosteric regulation of tryptophan synthase: Effects of $\mathrm{pH}$, temperature, and alphasubunit ligands on the equilibrium distribution of pyridoxal 5'-phosphate-L-serine intermediates. Biochemistry 35: 1872-1880.

Percudani R. and Peracchi A. 2003. A genomic overview of pyridoxal-phosphatedependent enzymes. EMBO Reports 4: 850-854.

Phillips R.S., Richter I., Gollnick P., Brzovic P., and Dunn M.F. 1991. Replacement of lysine 269 by arginine in Escherichia coli tryptophan indole-lyase accefts the formation and breakdown of quinonoid complexes. The Journal of Biological Chemistry 28: 1864218648.

Schneider G., Kack H., and Lindqzist Y. 2000. The manifold of vitamin $B_{6}$ dependent enzymes. Structure 8: R1-R6.

Siebert N., Cantre D., Eipel C., and Vollmar B. 2008. H2S contributes to the hepatic arterial buffer response and mediates vasorelaxation of the hepatic artery via activation of KATP channels. Am J Physiol Gastrointest Liver Physiol 295: G1266-G1273.

Steegborn C., Clausen T., Sonderman P., Jacob U., Worbs M., Marinkovic S., Huber R., and Wahl M.C. 1999. Kinetics and inhibition of recombinant human cystathionine $\gamma$ lyase. Journal of Biological Chemistry 274: 12675-12684.

Takagi H., Yoshioka K., Awano N., Nakamori S., and Ono B.I. 2003. Role of Saccharomyces cerevisiae serine O-acetyltransferase in cysteine biosynthesis. FEMS Microbiology Letters 218: 291-297.

Tan D., Barber M.J., and Ferreira G.C. 1998. The role of tyrosine 121 in cofactor binding of 5-aminolevulinate synthase. Protein Science 7: 1208-1213.

Taoka S., Ohja S., Shan X., Fruger W.D. and Banerjee R. 1998. Evidence for hememediated redox regulation of human cystathionine $\beta$-synthase activity. The Journal of Biological Chemistry 273: 25179-25184.

Thomas D. and Surdin-Kerjan Y. 1997. Metabolism of sulfur amino acids in Saccharomyces cerevisiae. Microbiology and Molecular Biology Reviews 61: 503-532.

Toney M.D. and Kirsch J.F. 1991. Tyrosine 70 fine-tunes the catalytic efficiency of aspartate aminotransferase. Biochemistry 30: 7456-7461.

Toney M.D. 2011. Controlling reaction specificity in pyridoxal phosphate enzymes. Biochimica et Biophysica Acta 1814: 1407-1418. 
Vacca R.A., Giannattasio S., Graber R., Sandmeier E., Marra E., and Christen P. 1997. Active-site Arg->Lys substitutions alter reaction and substrate specificity of aspartate aminotransferase. The Journal of Biological Chemistry 272: 21932-21937.

Wang J. and Hegele R.A. 2003. Genomic basis of cysthationinuria (MIM 219500) revealed by multiple mutations in cystathionine gamma-lyase (CTH). Hum Genet 112: 404-408.

Warenycia M.W., Goodwin L.R., Benishin C.G., Reiffenstein R.J., Francom D.M., Taylor J.D., and Dieken F.P. 1989. Acute hydrogen sulphide poisoning: demonstration of selective uptake of sulphide by the brainstem by measurement of brain sulphide levels. Biochemical Pharmacology 38: 973-981.

Wu G., Fang Y.Z., Yang S., Lupton J.R., Turner N.D. 2004. Glutathione metabolism and its implications for health. The Journal of Nutrition 134: 489-492.

Yamagata S. 1989. Roles of O-acetyl-L-homoserine sulfhydrylases in microorganisms. Biochimie 71: 1125-1143.

Yamagata S., D'Andrea R.J., Fujisaki A., Isaji M., and Nakamura K. 1993. Cloning and bacterial expression of the CYS3 gene encoding cystathionine $\gamma$-lyase of Saccharomyces cerevisiae and the physiochemical and enzymatic properties of the protein. Journal of Bacteriology 175: 4800-4808.

Yang G., Wu L., Jiang B., Yang W., Qi J., Cao K., Meng Q., Mustafa A.K., Mu W., Zhang S., Snyder S.H., and Wang R. 2008. H2S as a physiological vasorelaxant: hypertension in mice with deletion of cystathionine $\gamma$-lyase. Science 322: 587-590.

Yang G., Wu Y., Bryan S., Khaper N., Mani S., and Wang R. 2010. Cystathionine gamma-lyase deficiency and overproliferation of smooth muscle cells. Cardiovascular Research 86: 487-495.

Zhu W., Lin A., and Banerjee R. 2008. Kinetic properties of polymorphic variants and pathogenic mutants in human cystathionine $\gamma$-lyase. Biochemistry 47: 6226-6232. 